TRANSACTIONS OF THE

AMERICAN MATHEMATICAL SOCIETY

Volume 354, Number 2, Pages 571-612

S 0002-9947(01)02803-3

Article electronically published on September 18, 2001

\title{
A CLASSIFICATION OF HYPERPOLAR AND COHOMOGENEITY ONE ACTIONS
}

\author{
ANDREAS KOLLROSS
}

\begin{abstract}
An isometric action of a compact Lie group on a Riemannian manifold is called hyperpolar if there exists a closed, connected submanifold that is flat in the induced metric and meets all orbits orthogonally. In this article, a classification of hyperpolar actions on the irreducible Riemannian symmetric spaces of compact type is given. Since on these symmetric spaces actions of cohomogeneity one are hyperpolar, i.e. normal geodesics are closed, we obtain a classification of the homogeneous hypersurfaces in these spaces by computing the cohomogeneity for all hyperpolar actions. This result implies a classification of the cohomogeneity one actions on compact strongly isotropy irreducible homogeneous spaces.
\end{abstract}

\section{INTRODUCTION AND RESULTS}

An isometric action of a compact Lie group on a Riemannian manifold $M$ is called polar if there exists a closed, connected submanifold $\Sigma$ of $M$ that meets all orbits orthogonally. Such a submanifold is called a section; it is automatically totally geodesic in $M$. If the section is also flat, then the action is called hyperpolar. Typical examples of hyperpolar actions are the adjoint actions of compact Lie groups equipped with a bi-invariant metric, where the sections are the maximal tori, or, more generally, the isotropy actions of symmetric spaces. Other examples are the polar representations of compact Lie groups. They are also hyperpolar because the sections are linear subspaces. The irreducible polar representations were classified by [DADOK]. The main result of [DADOK $]$ is that all polar representations are orbit equivalent (i.e. have the same orbits) to isotropy representations of symmetric spaces. The principal orbits of polar representations are isoparametric submanifolds of $\mathrm{S}^{\mathrm{n}-1} \subset \mathbb{R}^{\mathrm{n}}$. More generally, the principal orbits of hyperpolar actions on symmetric spaces of the compact type are equifocal submanifolds [TT.

The main result of this article is the classification of hyperpolar actions on the irreducible Riemannian symmetric spaces of the compact type up to orbit equivalence. The problem of classifiying hyperpolar actions on symmetric spaces was posed in [HPTT1]. A classification and structure theory for hyperpolar actions with a fixed point on compact homogeneous Riemannian manifolds was developed in [HPTT2]. An important special case of independent interest is given by cohomogeneity one actions, i.e. actions whose principal orbits have codimension one.

Received by the editors October 10, 2000.

2000 Mathematics Subject Classification. Primary 53C35, 57S15.

Key words and phrases. Hyperpolar actions, cohomogeneity one actions, symmetric spaces, compact Lie groups.

Supported by Deutsche Forschungsgemeinschaft. 
As is shown in [HPTT1], cohomogeneity one actions on symmetric spaces of the compact type equipped with the metric induced by the Killing form are hyperpolar, i.e. normal geodesics are closed. Another special case was found by [HR]: If $H$ and $K$ are symmetric subgroups of a compact Lie group $G$, then the action of $H$ on $G / K$ and the action of $H \times K$ on $G$ are hyperpolar. These are the so-called Hermann actions. More generally, the actions of symmetric subgroups of $G \times G$ on $G$ are hyperpolar. We define here a symmetric subgroup $K$ of a compact Lie group $G$ to be a subgroup such that its Lie algebra is the fixed point set of an involutive automorphism of the Lie algebra of $G$.

The main results of this article are given in Theorems A and B. In the theorems, only actions on the simple compact Lie groups are given explicitly since, in order to classify the hyperpolar or cohomogeneity one actions on the irreducible symmetric spaces, it is sufficient to classify these actions on the simple compact Lie groups; because, if $K$ is a symmetric subgroup of the simple compact Lie group $G$, then $H \subset G$ acts hyperpolarly on $G / K$ if and only if $H \times K$ does on $G$, and these two actions have the same cohomogeneity.

Note that the cohomogeneity of a hyperpolar action on a symmetric space cannot be greater than the rank of the symmetric space, since a section is a flat, totally geodesic subspace.

The classification problem for hyperpolar actions can be formulated on the Lie algebra level. Therefore, the actions in Theorem A and Theorem B are only given up to "local conjugacy", i.e. up to isometries and covering maps of $G$ and up to connected components of the group acting. Similarly, "locally orbit equivalent" refers to the actions whose orbits are the same on the Lie algebra level, i.e. up to connected components and up to covering maps of $G$.

The first theorem gives the classification of the hyperpolar actions on the irreducible Riemannian symmetric spaces of the compact type.

Theorem A. Let $G$ be a connected simple compact Lie group, equipped with a bi-invariant metric. Let $U \subset G \times G$ be a closed connected subgroup that acts hyperpolarly and non-transitively on $G$ by

$$
\left(g_{1}, g_{2}\right) \cdot g=g_{1} g g_{2}^{-1}, \quad\left(g_{1}, g_{2}\right) \in U, g \in G .
$$

Then there is a closed subgroup $U^{\prime} \subset G \times G$ such that $U \subseteq U^{\prime}$, the $U$-action on $G$ and the $U^{\prime}$-action on $G$ are orbit equivalent and the $U^{\prime}$-action is locally conjugate to one of the following:

i) An action of a symmetric subgroup of $G \times G$.

ii) The $\varrho(H) \times \mathrm{SO}(\mathrm{n}-1)$-action on $\mathrm{SO}(\mathrm{n})$, where $H$ is a compact Lie group and the representation $\varrho: H \rightarrow \mathrm{SO}(\mathrm{n})$ is equivalent to the isotropy representation of a symmetric space of rank two.

iii) The $\varrho(H) \times \mathrm{S}\left(\mathrm{U}_{1} \times \mathrm{U}_{n-1}\right)$-action on $\mathrm{SU}(\mathrm{n})$, where $\varrho: H \rightarrow \mathrm{SU}(\mathrm{n})$ is a representation of the compact Lie group $H$ and $\theta$ a one-dimensional representation of $\mathrm{U}(1)$ such that $\varrho \otimes \theta: H \cdot \mathrm{U}(1) \rightarrow \mathrm{U}(\mathrm{n})$ is equivalent to the isotropy representation of a Hermitian symmetric space of rank two.

iv) The $\varrho(H) \times(\mathrm{Sp}(1) \times \mathrm{Sp}(\mathrm{n}-1))$-action on $\mathrm{Sp}(\mathrm{n})$, where $\varrho: H \rightarrow \mathrm{Sp}(\mathrm{n})$ is a representation of the compact Lie group $H$ and $\theta$ the standard representation of $\mathrm{Sp}(1)$ such that $\varrho \otimes \theta: H \cdot \mathrm{Sp}(1) \rightarrow \mathrm{SO}(4 \mathrm{n})$ is equivalent to the isotropy representation of a quaternionic-Kähler symmetric space of rank two.

v) The $H \times K$-action on $G$, where the triple $(H, G, K)$ is one of the following. 


\begin{tabular}{|c|c|c|}
\hline$H$ & $G$ & $K$ \\
\hline \hline $\mathrm{G}_{2}$ & $\mathrm{SO}(7)$ & $\mathrm{SO}(4) \times \mathrm{SO}(3)$ \\
\hline $\mathrm{G}_{2}$ & $\mathrm{SO}(7)$ & $\mathrm{G}_{2}$ \\
\hline $\mathrm{U}(3)$ & $\mathrm{SO}(7)$ & $\mathrm{G}_{2}$ \\
\hline $\mathrm{Spin}(9)$ & $\mathrm{SO}(16)$ & $\mathrm{SO}(14) \times \mathrm{SO}(2)$ \\
\hline $\mathrm{Sp}(\mathrm{n}) \mathrm{Sp}(1)$ & $\mathrm{SO}(4 \mathrm{n})$ & $\mathrm{SO}(4 \mathrm{n}-2) \times \mathrm{SO}(2)$ \\
\hline $\mathrm{SU}(3)$ & $\mathrm{G}_{2}$ & $\mathrm{SO}(4)$ \\
\hline $\mathrm{SU}(3)$ & $\mathrm{G}_{2}$ & $\mathrm{SU}(3)$ \\
\hline
\end{tabular}

TABLE 1. "Exceptional" cohomogeneity one actions

In particular, if the $U$-action on $G$ is not locally orbit equivalent to an action of a symmetric subgroup of $G \times G$, it is of cohomogeneity one.

The classification of cohomogeneity one actions is given in Theorem B. This generalizes the classifications of homogeneous hypersurfaces in spheres [HsL], complex [TAKAGI], quaternionic projective space D'ATRI] and the Cayley plane [WATA.

Theorem B. Let $G$ be a connected simple compact Lie group. Let $U \subset G \times G$ be a closed connected subgroup that acts with cohomogeneity one on $G$ by

$$
\left(g_{1}, g_{2}\right) \cdot g=g_{1} g g_{2}^{-1}, \quad\left(g_{1}, g_{2}\right) \in U, g \in G .
$$

Then $U$ is contained in a closed connected subgroup $U^{\prime} \subset G \times G$ such that the $U$-action and the $U^{\prime}$-action on $G$ are orbit equivalent and the $U^{\prime}$-action on $G$ is locally conjugate to one of the following:

i) A Hermann action of cohomogeneity one: i.e. the $K \times K$-action on $G$, where $K \subset G$ is a symmetric subgroup such that $\mathrm{rk}(\mathrm{G} / \mathrm{K})=1$, or the $H \times K$-action on $G$, where the triple $(H, G, K)$ is one of the following:

\begin{tabular}{|c|c|c|}
\hline$H$ & $G$ & $K$ \\
\hline \hline $\mathrm{SO}(\mathrm{p}+1)$ & $\mathrm{SU}(\mathrm{p}+1)$ & $\mathrm{S}\left(\mathrm{U}_{p} \times \mathrm{U}_{1}\right)$ \\
\hline $\mathrm{S}_{\left(\mathrm{U}_{2} \times \mathrm{U}_{2 \mathrm{n}-2}\right)}$ & $\mathrm{SU}(2 \mathrm{n})$ & $\mathrm{Sp}(\mathrm{n})$ \\
\hline $\mathrm{S}_{\left.\mathrm{U}_{3} \times \mathrm{U}_{2 \mathrm{n}-3}\right)}$ & $\mathrm{SU}(2 \mathrm{n})$ & $\mathrm{Sp}(\mathrm{n})$ \\
\hline $\mathrm{S}\left(\mathrm{U}_{\mathrm{a}+\mathrm{b}} \times \mathrm{U}_{1}\right)$ & $\mathrm{SU}(\mathrm{a}+\mathrm{b}+1)$ & $\mathrm{S}\left(\mathrm{U}_{\mathrm{a}} \times \mathrm{U}_{\mathrm{b}+1}\right)$ \\
\hline $\mathrm{SO}(\mathrm{a}+\mathrm{b})$ & $\mathrm{SO}(\mathrm{a}+\mathrm{b}+1)$ & $\mathrm{SO}(\mathrm{a}) \times \mathrm{SO}(\mathrm{b}+1)$ \\
\hline $\mathrm{Sp}(\mathrm{p}) \times \mathrm{Sp}(1)$ & $\mathrm{Sp}(\mathrm{p}+1)$ & $\mathrm{U}(\mathrm{p}+1)$ \\
\hline $\mathrm{Sp}(\mathrm{a}+\mathrm{b}) \times \mathrm{Sp}(1)$ & $\mathrm{Sp}(\mathrm{a}+\mathrm{b}+1)$ & $\mathrm{Sp}(\mathrm{a}) \times \mathrm{Sp}(\mathrm{b}+1)$ \\
\hline $\mathrm{SO}(2) \times \mathrm{SO}(2 \mathrm{n}-2)$ & $\mathrm{SO}(2 \mathrm{n})$ & $\mathrm{U}(\mathrm{n})$ \\
\hline $\mathrm{SO}(3) \times \mathrm{SO}(2 \mathrm{n}-3)$ & $\mathrm{SO}(2 \mathrm{n})$ & $\mathrm{U}(\mathrm{n})$ \\
\hline $\mathrm{SU}(6) \cdot \mathrm{SU}(2)$ & $\mathrm{E}_{6}$ & $\mathrm{~F}_{4}$ \\
\hline $\mathrm{Spin}(10) \cdot \mathrm{SO}(2)$ & $\mathrm{E}_{6}$ & $\mathrm{~F}_{4}$ \\
\hline $\mathrm{Sp}(3) \cdot \mathrm{Sp}(1)$ & $\mathrm{F}_{4}$ & $\mathrm{Spin}(9)$ \\
\hline
\end{tabular}

TABle 2. Cohomogeneity one Hermann actions

ii) A $\sigma$-action of cohomogeneity one, i.e. the adjoint action of $\mathrm{SU}(2)$, or the action of $\{(g, \sigma(g)) \mid g \in G\}$ on $\mathrm{SU}(3)$, where $\sigma$ is an outer automorphism of $\mathrm{SU}(3)$.

iii) An action as described in parts ii), iii), iv) or v) of Theorem $A$. 
We have actually obtained a classification of cohomogeneity one actions on all compact strongly isotropy irreducible homogeneous spaces (see the remark after Definition 1.12).

This article is organized as follows.

In the first section we give definitions and review some preliminary results. The classification problem is reduced to the problem of finding hyperpolar actions of maximal non-transitive groups on the simple compact Lie groups.

In the second section, the classification of the hyperpolar actions is carried out. Lower bounds on the dimension of groups acting hyperpolarly are obtained and, using these necessary conditions, many groups can be shown not to act hyperpolarly. For the remaining actions, slice representations are obtained in order to decide whether these actions are polar or to compute the cohomogeneity. It then turns out that all actions not excluded by these conditions are Hermann actions or cohomogeneity one actions.

In the third section, the cohomogeneities of the Hermann actions and $\sigma$-actions on the irreducible symmetric spaces of compact type are computed. This information is needed to complete the classification of the cohomogeneity one actions, i.e. to find out which of these actions are of cohomogeneity one.

The polar actions on compact rank one symmetric spaces were recently classified by $[\mathrm{PTH}$. It remains an interesting open question if there are polar actions on the symmetric spaces of higher rank which are not hyperpolar.

The author would like to thank Ernst Heintze and Wolfgang Ziller for many useful comments and to thank Robert Bock and Jens Heber for discussions.

\section{Preliminaries}

Definition 1.1. Let $G$ be a compact Lie group and $M$ a connected, complete Riemannian $G$-manifold. A connected, closed, smooth submanifold $\Sigma$ is called a section if it meets all orbits, i.e. $G \Sigma=M$ and for each $x \in M$, the tangent space $\mathrm{T}_{\mathrm{x}} \Sigma$ is included in $\nu_{x}(G x)=\left(\mathrm{T}_{\mathrm{x}}(\mathrm{Gx})\right)^{\perp}$.

The action of $G$ on $M$ is called polar if there exists a section. It is called hyperpolar if it is polar and the section is flat in the induced metric.

Since the notion hyperpolarity is defined for the category of Riemannian $G$ manifolds, we are naturally lead to consider the concept of isomorphism in this category:

Definition 1.2. Let $M_{1}$ be a Riemannian $G_{1}$-manifold and let $M_{2}$ be a Riemannian $G_{2}$-manifold. Then $M_{1}$ and $M_{2}$ are called conjugate if there is an isomorphism $\Phi: G_{1} \rightarrow G_{2}$ and an isometry $F: M_{1} \rightarrow M_{2}$ such that $\Phi(g) \cdot F(x)=F(g \cdot x)$ for all $g \in G_{1}$ and all $x \in M_{1}$.

We are mainly interested in the geometry of the orbits, therefore we also use a weaker equivalence relation, where we regard two actions as equivalent if there is an isometry that maps orbits to orbits.

Definition 1.3. Let $M_{1}$ be a Riemannian $G_{1}$-manifold and let $M_{2}$ be a Riemannian $G_{2}$-manifold. Then $M_{1}$ and $M_{2}$ are called orbit equivalent if there is an isometry $F: M_{1} \rightarrow M_{2}$ such that $F\left(G_{1} x\right)=G_{2} F(x)$ for all $x \in M_{1}$.

Definition 1.4. Let $G$ be a compact Lie group and $K$ a closed subgroup. The pair $(G, K)$ is called a symmetric pair if there exists an involution (i.e. an automorphism 
of order 2) of $G$ such that $G_{0}^{\sigma} \subseteq K \subseteq G^{\sigma}$, where $G^{\sigma}$ and $G_{0}^{\sigma}$ are the fixed point set of $\sigma$ and its connected component, respectively.

Every symmetric space $M$ may be presented as $G / K$, where $G$ is the isometry group of $M$ and $K$ is a symmetric subgroup of $G$. Conversely, if $(G, K)$ is a symmetric pair, $G / K$ is a symmetric space if it is equipped with an appropriate metric. If $M$ is an irreducible Riemannian symmetric space of compact type, then either $M$ is a simple, compact, connected Lie group (symmetric space of type II or group type), or $M=G / K$, where $G$ is a simple, compact, connected Lie group and $K$ a symmetric subgroup of $G$ (symmetric space of type III).

Definition 1.5. Let $G$ be a compact connected semisimple Lie group and $U$ a subgroup of $G \times G$. We define the $U$-action on $G$ by

$$
\left(g_{1}, g_{2}\right) \cdot g=g_{1} g g_{2}^{-1}, \quad\left(g_{1}, g_{2}\right) \in U, g \in G .
$$

If $U=H \times K$, where $H$ and $K$ are symmetric subgroups of $G$, then the $H \times K$ action on $G$ is called a Hermann action. If $\sigma$ is an automorphism of $G$ and $U=$ $\{(g, \sigma(g)) \mid g \in G\}$, then the $U$-action on $G$ is called the $\sigma$-action. If $\sigma=\operatorname{id}_{G}$, then the $\sigma$-action is called the adjoint action of $G$.

Since, as a symmetric space, $G$ may be presented as $G \cong G \times G / \Delta G$ and $U=$ $\{(g, \sigma(g)) \mid g \in G\}$ is the fixed point set of the involution $(g, h) \mapsto\left(\sigma^{-1}(h), \sigma(g)\right)$ of $G \times G, \sigma$-actions may be viewed as Hermann actions on $G \times G$. Both $\sigma$-actions and Hermann actions can be described as actions of symmetric subgroups of $G \times G$ on $G$.

An important notion in the theory of Riemannian $G$-manifolds is the so-called slice representation; see e.g. [J̈̈NICH]:

Definition 1.6. Let $M$ be a Riemannian $G$-manifold and let $G_{x}$ be the isotropy group at $x$. The differential of the action of $G_{x}$ defines a linear representation of $G_{x}$ on $\mathrm{T}_{\mathrm{x}} \mathrm{M}$ called the isotropy representation. The tangent space $\mathrm{T}_{\mathrm{x}} \mathrm{Gx}$ and the normal space $\nu_{x} G x$ to the orbit of $x$ are invariant subspaces of the isotropy representation. The restriction of the isotropy representation to $\nu_{x} G x$ is called the slice representation at $x$.

The slice representation is particularly useful for our classification of hyperpolar actions because the polarity of an action is inherited by its slice representations.

Theorem 1.7. Let $M$ be a Riemannian $G$-manifold. Then the cohomogeneity of a slice representation equals the cohomogeneity of the $G$-action on $M$. If the action on $M$ is polar, then for all $x \in M$ the slice representation at $x$ is polar.

Proof. See PT1, Theorem 4.6.

We will use this theorem to prove that certain actions are not hyperpolar by showing that they have non-polar slice representations and also to show that certain actions have cohomogeneity one. For this purpose, we will frequently use the results of DADOK, where polar representations were classified.

In [HPTT1], an algebraic criterion for hyperpolarity was obtained:

Theorem 1.8. Let $G$ be a compact, connected, semi-simple Lie group with the biinvariant metric induced from the negative of the Killing form on $\mathfrak{g}$. Let $U$ be a closed subgroup of $G \times G$. Then the following are equivalent:

i) The $U$-action on $G$ is hyperpolar. 
ii) There exists $g \in G$ such that $g^{-1} \nu_{g}(U g)$ is abelian.

iii) The normal space $\nu_{e}(U e)$ contains a $k$-dimensional abelian subalgebra, where $k$ is the cohomogeneity of the $U$-action on $G$.

As an immediate and important consequence of this criterion, the problem of finding all hyperpolar actions on $G$ is reduced to a problem on the Lie algebra level. Also the hyperpolarity of the following well-known examples can be shown using the preceding theorem; see [HPTT1], Section 3.1:

Proposition 1.9. Hermann actions, $\sigma$-actions and cohomogeneity one actions on irreducible symmetric spaces of the compact type are hyperpolar.

Now we will reformulate the problem of finding all hyperpolar and cohomogeneity one actions on the compact irreducible symmetric spaces. As a first step, we note that the classification of hyperpolar actions on $G / K$ may be reduced to that of $G$.

Proposition 1.10. Let $(G, K)$ be a symmetric pair, $M=G / K$ the corresponding symmetric space and $H$ a closed subgroup of $G$. Then the $H$-action on $M$ is hyperpolar if and only if the $H \times K$-action on $G$ is hyperpolar.

Proof. See HPTT1, Proposition 2.11

Thus, concerning the classification of hyperpolar actions, it is not necessary to distinguish between the $H \times K$-action on $G$ and the $H$-action on $G / K$ in the situation described in Proposition 1.10, and in the following we will switch between these two points of view without mention.

Another important fact is the following maximality property of hyperpolar actions. By this property, we may restrict our attention to maximal subgroups of groups that act transitively on symmetric spaces, as far as classification up to orbit equivalence is concerned.

Theorem 1.11. Let $M=G / K$ be a compact, connected, irreducible symmetric space and $H \subset L \subset G$ closed, connected subgroups. If the $H$-action on $M$ is hyperpolar, then the L-action on $M$ is transitive or is orbit equivalent to the $H$ action.

Proof. This was originally proved in [HPTT1, Corollary 3.14, under an additional hypothesis, which was recently removed in $\underline{\mathrm{HL}}$, Corollary D.

Theorem 1.11 motivates the following definition.

Definition 1.12. Let $G$ be a connected Lie group and $H \subset G$ a closed, connected subgroup. We call $H$ a maximal connected subgroup of $G$, if for every closed, connected subgroup $H^{\prime}$ with $H \subseteq H^{\prime} \subseteq G$ either $H=H^{\prime}$ or $H^{\prime}=G$. Let $U \subset G \times G$ be a closed, connected subgroup. We call $U$ a maximal connected nontransitive subgroup of $G \times G$, if the $U$-action on $G$ is not transitive and for every closed, connected subgroup $U^{\prime}$ with $U \subseteq U^{\prime} \subseteq G \times G$ either $U=U^{\prime}$ or $U^{\prime}$ acts transitively on $G$.

Remark. Proposition 1.10 and Theorem 1.11 together imply that, in order to classify the hyperpolar actions on the irreducible symmetric spaces of the compact type up to orbit equivalence, we may restrict ourselves to decide which maximal connected non-transitive subgroups of $G \times G$ act hyperpolarly on $G$ for all simple compact Lie groups $G$; because we have that if $K$ is a symmetric subgroup of $G$ and the closed subgroup $H$ acts hyperpolarly on $G / K$, then there is a group $H^{\prime}$ having 
the same orbits as $H$ on $G / K$, such that $H \subseteq H^{\prime} \subset G$ and $H^{\prime} \times K$ is a maximal connected non-transitive subgroup of $G$ that acts hyperpolarly on $G$. (We assume that $K$ is connected.) This follows from the fact that the connected symmetric subgroups of the simple compact Lie groups are maximal connected. An analogous argument holds if $G / K$ is a compact strongly isotropy irreducible homogeneous space, since in this case $K \subset G$ is a maximal connected subgroup.

The next proposition shows that we have to consider the subgroups of $G \times G$ only up to conjugacy. This follows from the fact that $G$ is a homogeneous space on which $G \times G$ acts transitively.

Proposition 1.13. Let $G$ be a compact Lie group equipped with a bi-invariant metric. Let $U$ be a subgroup of $G \times G$. Let $a, b \in G$. Consider the subgroup

$$
U^{\prime}=\left\{\left(a g_{1} a^{-1}, b g_{2} b^{-1}\right) \mid\left(g_{1}, g_{2}\right) \in U\right\} .
$$

Then the $U$-action on $G$ and the $U^{\prime}$-action on $G$ are conjugate via the isometry

$$
F: G \rightarrow G, g \mapsto a g b^{-1} .
$$

However, one should be aware of the fact that if an outer automorphism of $G \times G$ is applied to $U \subset G \times G$, the resulting actions are not conjugate in general; see e.g. Theorem 3.2 We will not distinguish the order of the two factors of $G \times G$ in the following, because the action of $U \subset G \times G$ and $\left\{\left(g_{2}, g_{1}\right) \mid\left(g_{1}, g_{2}\right) \in U\right\}$ on $G$ are conjugate via the isometry $g \mapsto g^{-1}$.

Finally, we can formulate the following classification problem:

For all simple compact Lie groups $G$, determine the conjugacy classes of maximal connected non-transitive subgroups $U \subset G \times G$ and decide which ones act hyperpolarly on $G$.

This problem will be solved in section 2 .

Cohomogeneity one actions are a special case of hyperpolar actions, thus we will obtain a classification of cohomogeneity one actions on the compact irreducible symmetric spaces with little extra effort. Since it will turn out that hyperpolar actions which are not of cohomogeneity one are orbit equivalent to Hermann actions or $\sigma$-actions, it is sufficient to compute the cohomogeneities for the Hermann and $\sigma$-actions. This is done in section 3 .

Since the above problem can be formulated entirely in terms of Lie algebras, we may state our results using the following definition, which defines two actions on two locally isomorphic Lie groups to be locally conjugate or locally orbit equivalent if the corresponding actions on the universal cover are conjugate or orbit equivalent, respectively.

Definition 1.14. Let $G_{1}$ and $G_{2}$ be two locally isomorphic compact connected semisimple Lie groups, i.e. there exist a simply connected compact Lie group $\tilde{G}$ and epimorphisms

$$
\pi_{1}: \tilde{G} \rightarrow G_{1} \text { and } \pi_{2}: \tilde{G} \rightarrow G_{2}
$$

that are universal coverings. Let $U_{1} \subset G_{1} \times G_{1}$ and $U_{2} \subset G_{2} \times G_{2}$ be closed subgroups. The $U_{1}$-action on $G_{1}$ and the $U_{2}$-action on $G_{2}$ are called locally orbit equivalent if the $\left(\tilde{U}_{1}\right)_{0}$-action on $\tilde{G}$ and the $\left(\tilde{U}_{2}\right)_{0}$-action on $\tilde{G}$ are orbit equivalent, where we define:

$$
\tilde{U}_{i}=\left\{(g, h) \in \tilde{G} \times \tilde{G} \mid\left(\pi_{i}(g), \pi_{i}(h)\right) \in U_{i}\right\}, \quad i=1,2 .
$$


The $U_{1}$-action on $G_{1}$ and the $U_{2}$-action on $G_{2}$ are called locally conjugate if the $\left(\tilde{U}_{1}\right)_{0}$-action on $\tilde{G}$ and the $\left(\tilde{U}_{2}\right)_{0}$-action on $\tilde{G}$ are conjugate.

By Theorem 1.8, we have that two locally orbit equivalent actions are either both hyperpolar or both not hyperpolar.

\section{The Classification of Hyperpolar Actions}

In this section we will solve the problem formulated at the end of the preceding section, i.e. for all simple compact Lie groups $G$, we find those connected subgroups of $G \times G$ that act hyperpolarly on $G$ and are maximal with respect to this property. In 2.1, we review some results of Dynkin, concerning the maximal subgroups of compact Lie groups. Since the section of a hyperpolar action on a symmetric space $M$ is a totally geodesic flat submanifold of $M$, its dimension is less or equal the rank of $M$. This fact is used in 2.2 to obtain lower bounds on the dimensions of the groups that act hyperpolarly on $M$. The main result of 2.2 is that if $H \times K$ acts hyperpolarly on $G=\mathrm{SU}(\mathrm{n}), \mathrm{SO}(\mathrm{n}), \mathrm{Sp}(\mathrm{n})$, then, with one exception, $H \subset G$ is a symmetric subgroup or $K \subset G$ is a symmetric subgroup. In 2.3, certain actions of non-symmetric subgroups of $G \times G$ which cannot be excluded by dimension conditions are examined. This is done by computing slice representations. It turns out that these actions are either not hyperpolar or of cohomogeneity one. Finally, in 2.4 the classification is completed. The remaining actions not excluded in 2.2 or 2.3 transitive subgroups and exceptional groups are considered.

2.1. Maximal Subgroups of Compact Lie Groups. The maximal connected subgroups of compact Lie groups are given by the following theorems, the first of which is a slight generalization of Theorem 15.1, p. 235 in [DYN1].

Theorem 2.1. Let

$$
\mathfrak{g}=\mathfrak{g}_{0} \oplus \mathfrak{g}_{1} \oplus \cdots \oplus \mathfrak{g}_{s}
$$

be a decomposition of the Lie algebra $\mathfrak{g}$ into a direct sum of ideals, where $\mathfrak{g}_{0}$ is abelian and $\mathfrak{g}_{1}, \ldots, \mathfrak{g}_{s}$ are simple. Let $\mathfrak{a}$ be a maximal subalgebra of $\mathfrak{g}$. Then either $\mathfrak{a}$ is equal to

$$
\bigoplus_{\substack{k=0 \\ k \neq i}}^{s} \mathfrak{g}_{k} \oplus \tilde{\mathfrak{g}}
$$

for an index $i \in\{0, \ldots, s\}$ and a maximal subalgebra $\tilde{\mathfrak{g}} \subset \mathfrak{g}_{i}$, or $\mathfrak{a}$ equals

$$
\bigoplus_{\substack{k=0 \\ k \neq i, j}}^{s} \mathfrak{g}_{k} \oplus \mathfrak{g}(i, j, \sigma),
$$

for two indices $i, j \in\{1, \ldots, s\}, i \neq j$, and an isomorphism $\sigma: \mathfrak{g}_{i} \rightarrow \mathfrak{g}_{j}$, where we define $\mathfrak{g}(i, j, \sigma)=\left\{X+\sigma(X) \mid X \in \mathfrak{g}_{i}\right\}$.

By Theorem [2.1, to find the maximal subgroups of compact Lie groups, it is sufficient to know the maximal subgroups of the simple compact Lie groups. For the classical groups, this problem was solved in [DYN2]. 
Theorem 2.2. Let $H$ be a maximal connected subgroup of $\mathrm{SO}(\mathrm{n})$. Then $H$ is conjugate (in $\mathrm{O}(\mathrm{n}))$ to one of the following:

\begin{tabular}{|r|c|l|}
\hline i) & $\mathrm{SO}(\mathrm{k}) \times \mathrm{SO}(\mathrm{n}-\mathrm{k})$ & $1 \leq k \leq n-1$ \\
\hline ii $)$ & $\mathrm{SO}(\mathrm{p}) \otimes \mathrm{SO}(\mathrm{q})$ & $p q=n, 3 \leq p \leq q$ \\
\hline iii $)$ & $\mathrm{U}(\mathrm{k})$ & $2 k=n$ \\
\hline iv $)$ & $\mathrm{Sp}(\mathrm{p}) \otimes \mathrm{Sp}(\mathrm{q})$ & $4 p q=n$ \\
\hline v) & $\varrho(H)$ & $H$ simple, $\varrho \in \operatorname{Irr}_{\mathbb{R}}(\mathrm{H}), \operatorname{deg} \varrho=n$ \\
\hline
\end{tabular}

Let $H$ be a maximal connected subgroup of $\mathrm{SU}(\mathrm{n})$. Then $H$ is conjugate to one of the following:

\begin{tabular}{|r|c|l|}
\hline i) & $\mathrm{SO}(\mathrm{n})$ & \\
\hline ii $)$ & $\mathrm{Sp}(\mathrm{m})$ & $2 m=n$ \\
\hline iii $)$ & $\mathrm{S}\left(\mathrm{U}_{k} \times \mathrm{U}_{n-k}\right)$ & $1 \leq k \leq n-1$ \\
\hline iv $)$ & $\mathrm{SU}(\mathrm{p}) \otimes \mathrm{SU}(\mathrm{q})$ & $p q=n, p \geq 3, q \geq 2$ \\
\hline $\mathrm{v})$ & $\varrho(H)$ & $H$ simple $, \varrho \in \operatorname{Irr}_{\mathbb{C}}(\mathrm{H}), \operatorname{deg} \varrho=n$ \\
\hline
\end{tabular}

Let $H$ be a maximal connected subgroup of $\mathrm{Sp}(\mathrm{n})$. Then $H$ is conjugate to one of the following:

\begin{tabular}{|r|c|l|}
\hline i) & $\mathrm{U}(\mathrm{n})$ & \\
\hline ii) & $\mathrm{Sp}(\mathrm{k}) \times \mathrm{Sp}(\mathrm{n}-\mathrm{k})$ & $1 \leq k \leq n-1$ \\
\hline iii) & $\mathrm{SO}(\mathrm{p}) \otimes \mathrm{Sp}(\mathrm{q})$ & $p q=n, p \geq 3, q \geq 1$ \\
\hline iv $)$ & $\varrho(H)$ & $H$ simple, $\varrho \in \operatorname{Irr}_{\mathbb{H}}(\mathrm{H}), \operatorname{deg} \varrho=2 n$ \\
\hline
\end{tabular}

By $\operatorname{Irr}_{\mathbb{R}}(\mathrm{H}), \operatorname{Irr}_{\mathbb{C}}(\mathrm{H}), \operatorname{Irr}_{\mathbb{H}}(\mathrm{H})$ we denote the irreducible representations of $H$ of real, complex and quaternionic type, respectively.

Remarks. Not all of the subgroups listed above are maximal connected subgroups. Some groups are not proper subgroups and there are some inclusions which are determined in [DYN2]. But for our purposes the information given in the above theorem is sufficient. The maximal subalgebras of the exceptional Lie algebras are classified in DYN1]. Note also that there are subgroups of $\mathrm{SO}(2 \mathrm{n})$, which are conjugate in $\mathrm{O}(2 \mathrm{n})$, but not in $\mathrm{SO}(2 \mathrm{n})$.

We will proceed as follows with the classification. Let $G$ be a simple compact connected Lie group. The maximal connected subgroups of $G \times G$ are, by Theorem 2.1 above,

$$
H_{1} \times G, \quad G \times H_{2}, \quad \Delta^{\sigma} G=\{(g, \sigma(g)) \mid g \in G\},
$$

where $H_{i}$ run through all maximal connected subgroups of $G$ and $\sigma$ runs through all automorphisms of $G$. The first two types of subgroups act transitively on $G$, so we have to consider their maximal connected subgroups. Since all subgroups which have $G$ as one factor act transitively, we arrive at the groups of the form $H \times K$, where both $H$ and $K$ are maximal connected subgroups of $G$. Now there are two alternatives: Either $H \times K$ acts transitively on $G$, then we have to consider maximal connected subgroups of $H \times K$; or $H \times K$ does not act transitively on $G$, then we have to find out if the $H \times K$-action on $G$ is hyperpolar or not.

The subgroups $\Delta^{\sigma} G$ correspond to the $\sigma$-actions, which are hyperpolar. 
2.2. Dimension Conditions. In the following, we will deduce and apply some necessary conditions on the dimensions of groups which act hyperpolarly. First we observe that there is a lower bound for the dimension of such groups. If $U$ acts hyperpolarly on the symmetric space $M$, the following inequality holds:

$$
\operatorname{dim}(U) \geq \operatorname{dim}(M)-\operatorname{rk}(\mathrm{M})
$$

because $\operatorname{dim}(U)$ is an upper bound for the dimension of an orbit of $U$, and a hyperpolar action on $M$ has at most cohomogeneity $\mathrm{rk}(\mathrm{M})$. In particular, if $H \times K$ acts hyperpolarly on $G$, we have

$$
\operatorname{dim}(H)+\operatorname{dim}(K) \geq \operatorname{dim}(G)-\operatorname{rk}(\mathrm{G}) .
$$

To obtain a necessary condition on the dimension of $H$, we replace $\operatorname{dim}(K)$ by the maximal dimension of a proper closed subgroup of $G$ :

$$
\operatorname{dim}(H) \geq m(G)-\operatorname{rk}(\mathrm{G})
$$

where we define

$$
m(G)=\min \{\operatorname{dim}(G / K) \mid K \subset G \text { proper closed subgroup }\} .
$$

We take the values of $m(G)$ for the simple compact Lie groups from [MANN] (see Table 3) where subgroups of maximal dimension are also given.

\begin{tabular}{|c|c|c|}
\hline$G$ & $m(G)$ & $H_{\max }$ \\
\hline \hline $\mathrm{SU}(\mathrm{n}), \mathrm{n} \neq 4$ & $2(n-1)$ & $\left.\mathrm{S}_{1} \mathrm{U}_{1} \times \mathrm{U}_{n-1}\right)$ \\
\hline $\mathrm{SO}(\mathrm{n})$ & $n-1$ & $\mathrm{SO}(\mathrm{n}-1)$ \\
\hline $\mathrm{Sp}(\mathrm{n}), \mathrm{n} \geq 2$ & $4(n-1)$ & $\mathrm{Sp}(1) \times \mathrm{Sp}(\mathrm{n}-1)$ \\
\hline $\mathrm{G}_{2}$ & 6 & $\mathrm{SU}(3)$ \\
\hline $\mathrm{F}_{4}$ & 16 & $\mathrm{Spin}(9)$ \\
\hline $\mathrm{E}_{6}$ & 26 & $\mathrm{~F}_{4}$ \\
\hline $\mathrm{E}_{7}$ & 54 & $\mathrm{E}_{6} \cdot \mathrm{SO}(2)$ \\
\hline $\mathrm{E}_{8}$ & 112 & $\mathrm{E}_{7} \cdot \mathrm{SU}(2)$ \\
\hline
\end{tabular}

TABLE 3. Subgroups of maximal dimension

Remarks. The restriction $n \neq 4$ for $\mathrm{SU}(\mathrm{n})$ is imposed because a subgroup of maximal dimension of $\mathrm{SU}(4) \cong \operatorname{Spin}(6)$ is not $\mathrm{S}\left(\mathrm{U}_{1} \times \mathrm{U}_{3}\right)$, but $\mathrm{Sp}(2) \cong \operatorname{Spin}(5)$. As a first consequence, we can see from the table above that for simple $G$, no proper subgroup of $G$ can act hyperpolarly on $G$, because $m(G)>\operatorname{rk}(\mathrm{G})$ for all simple $G$.

Since a subgroup $H$ of a classical Lie group $(\mathrm{SO}(\mathrm{n}), \mathrm{SU}(\mathrm{n})$ or $\mathrm{Sp}(\mathrm{n}))$ is given by a representation $\varrho$ of $H$ of the corresponding (real, complex or quaternionic) type and degree ( $n$, or $2 n$ for $\mathrm{Sp}(\mathrm{n}))$, we may reformulate condition (2.5) in terms of the degree of $\varrho$. If $H$ is a subgroup of $\mathrm{SO}(\mathrm{n})$ given by $\varrho$, the condition becomes

$$
\operatorname{dim} H \geq \operatorname{deg} \varrho-1-\left\lfloor\frac{\operatorname{deg} \varrho}{2}\right\rfloor,
$$

which is equivalent to the inequality

$$
2 \operatorname{dim} H \geq \operatorname{deg} \varrho-2 .
$$

Similar conditions are obtained for subgroups of $\mathrm{SU}(\mathrm{n})$ and $\mathrm{Sp}(\mathrm{n})$. We summarize these remarks by the following lemma. Note that we assume here that $\mathrm{Sp}(\mathrm{n})$ is a subgroup of $\mathrm{SU}(2 \mathrm{n})$. 
Lemma 2.3. Let $G$ be one of the classical Lie groups $\mathrm{SO}(\mathrm{n}), \mathrm{SU}(\mathrm{n})$ or $\mathrm{Sp}(\mathrm{n})$. Let $H$ and $K$ be closed subgroups of $G$. Let $\varrho$ be the representation of $H$ which is defined by restriction of the standard representation of $G$ to $H$. If $H \times K$ acts hyperpolarly on $G$, then $H$ and $\varrho$ satisfy the following conditions:

$$
\begin{aligned}
2 \operatorname{dim} H \geq \operatorname{deg} \varrho-2 & \text { if } G=\mathrm{SO}(\mathrm{n}), \\
\operatorname{dim} H \geq \operatorname{deg} \varrho-1 & \text { if } G=\mathrm{SU}(\mathrm{n}), \\
\operatorname{dim} H \geq \frac{3}{2} \operatorname{deg} \varrho-4 & \text { if } G=\mathrm{Sp}(\mathrm{n}) .
\end{aligned}
$$

For any simple compact Lie group $H$ there are only finitely many irreducible representations $\varrho$ of $H$ such that the corresponding conditions (2.6), (2.7) or (2.8) hold. By the following three lemmas we will determine all such representations. We start by excluding some fundamental representations.

Lemma 2.4. The following fundamental representations of simple Lie groups $H$ do not satisfy condition (2.6) if $\varrho$ is of real type, (2.7) if $\varrho$ is of complex type or (2.8) if $\varrho$ is of quaternionic type, respectively. Thus the corresponding subgroups $\varrho(H)$ of the respective classical Lie groups $G$ do not act hyperpolarly on any quotient $G / K$.

\begin{tabular}{|c|c|c|c|}
\hline$H$ & $\varrho_{k}$ & $\operatorname{deg} \varrho_{k}$ & Range \\
\hline \hline $\mathrm{A}_{n}$ & $\varrho_{k}, k=3, \ldots, n-2$ & $\left(\begin{array}{c}n+1 \\
k\end{array}\right)$ & $n \geq 8$ \\
\hline $\mathrm{B}_{n}$ & $\varrho_{k}, k=3, \ldots, n-1$ & $\left(\begin{array}{c}2 n+1 \\
k\end{array}\right)$ & $n \geq 4$ \\
\hline $\mathrm{C}_{n}$ & $\varrho_{k}, k=3, \ldots, n$ & $\left(\begin{array}{c}2 n \\
k\end{array}\right)-\left(\begin{array}{c}2 n \\
k-2\end{array}\right)$ & $n \geq 6$ \\
\hline $\mathrm{D}_{n}$ & $\varrho_{k}, k=3, \ldots, n-2$ & $\left(\begin{array}{c}2 n \\
k\end{array}\right)$ & $n \geq 5$ \\
\hline $\mathrm{B}_{n}$ & $\varrho_{n}$ & $2^{n}$ & $n \geq 9$ \\
\hline $\mathrm{D}_{n}$ & $\varrho_{n-1}, \varrho_{n}$ & $2^{n-1}$ & $n \geq 9$ \\
\hline
\end{tabular}

Proof. The numbering of the fundamental representations is taken from [TITS, where the types of fundamental representations are also given.

With the exception of $\varrho_{(n+1) / 2}$, the fundamental representations of $\mathrm{A}_{n}$ are of complex type, thus we have to check condition (2.7). It clearly suffices to verify the case $k=3$. The statement holds for $n=8$ and we have $\operatorname{deg} \varrho_{3}\left(\mathrm{~A}_{\mathrm{n}+1}\right)=$ $\left(\begin{array}{c}\mathrm{n}+2 \\ 3\end{array}\right)=\left(\begin{array}{c}\mathrm{n}+1 \\ 3\end{array}\right)+\left(\begin{array}{c}\mathrm{n}+1 \\ 2\end{array}\right)=\operatorname{deg} \varrho_{3}\left(\mathrm{~A}_{\mathrm{n}}\right)+\frac{1}{2} \mathrm{n}(\mathrm{n}+1)$. On the other hand, we have $\operatorname{dim} \mathrm{A}_{\mathrm{n}+1}=\operatorname{dim} \mathrm{A}_{\mathrm{n}}+2 \mathrm{n}+3$, and $\frac{1}{2} n(n+1)>2 n+3$ if $n \geq 8$.

It may easily be verified that the representations $\varrho_{(n+1) / 2}$ do not fulfill the respective conditions.

Similar arguments apply to the fundamental representations $\varrho_{3}, \ldots, \varrho_{n-1}$ of $\mathrm{B}_{\mathrm{n}}$ and $\varrho_{3}, \ldots, \varrho_{n-2}$ of $\mathrm{D}_{\mathrm{n}}$. In these cases it is again sufficient to consider the representation $\varrho_{3}=\Lambda^{3}\left(\varrho_{1}\right)$ of $\mathrm{SO}(\mathrm{N})$. For $\mathrm{SO}(\mathrm{N})$ we have $\operatorname{deg} \varrho_{3}=\left(\begin{array}{c}N \\ 3\end{array}\right)=$ $\frac{1}{6} N(N-1)(N-2) \geq \frac{7}{3} \operatorname{dim}(\mathrm{SO}(\mathrm{N}))$ for $N \geq 9$, from which it follows that $\operatorname{deg} \varrho_{3}>$ $2 \operatorname{dim}(\mathrm{SO}(\mathrm{N}))+2$.

For $\mathrm{C}_{\mathrm{n}}$, we have to estimate the degree of $\varrho_{k}$ for arbitrary $k \in\{3, \ldots, n\}$. We have

$$
\operatorname{deg} \varrho_{k}=\left(\begin{array}{c}
2 n \\
k
\end{array}\right)-\left(\begin{array}{c}
2 n \\
k-2
\end{array}\right)=\left(\begin{array}{c}
2 n \\
k
\end{array}\right)\left(1-\frac{(k-1) k}{(2 n-k+1)(2 n-k+2)}\right) .
$$


By setting $k=4$ in the left factor and $k=n$ in the right, one can estimate the last term from below and one gets that conditions (2.6) and (2.8) are not satisfied for $\varrho_{4}, \ldots, \varrho_{n}$ if $n \geq 6$. It is easy to see that the fundamental representations $\varrho_{3}$, which are of quaternionic type, violate condition (2.8) if $n \geq 6$.

For the spin and of half-spin representations of $B_{n}$ and $D_{n}$, respectively, one may use a simple induction proof.

Lemma 2.5. The following representations of simple Lie groups $H$ do not satisfy condition (2.6) if $\varrho$ is of real type, (2.7) if $\varrho$ is of complex type or (2.8) if $\varrho$ is of quaternionic type, respectively. Thus the corresponding subgroups $\varrho(H)$ of the respective classical Lie groups $G$ do not act hyperpolarly on any quotient $G / K$.

\begin{tabular}{|c|c|c|c|}
\hline$H$ & $\left(c_{1}, \ldots, c_{n}\right)$ & $\operatorname{deg} \varrho$ & Range \\
\hline \hline $\mathrm{A}_{\mathrm{n}}$ & $(1,1,0, \ldots, 0,0)$ & $\frac{1}{3} n(n+1)(n+2)$ & $n \geq 3$ \\
\hline $\mathrm{A}_{\mathrm{n}}$ & $(0,1,0, \ldots, 0,1)$ & $\frac{1}{2}(n-1)(n+1)(n+2)$ & $n \geq 4$ \\
\hline $\mathrm{A}_{\mathrm{n}}$ & $(0,1,0, \ldots, 0,1,0)$ & $\frac{1}{4}(n-2)(n+1)^{2}(n+2)$ & $n \geq 4$ \\
\hline $\mathrm{A}_{\mathrm{n}}$ & $(3,0,0, \ldots, 0,0)$ & $\frac{1}{6}(n+1)(n+2)(n+3)$ & $n \geq 2$ \\
\hline $\mathrm{A}_{\mathrm{n}}$ & $(0,2,0, \ldots, 0,0)$ & $\frac{1}{12} n(n+1)^{2}(n+2)$ & $n \geq 4$ \\
\hline $\mathrm{B}_{\mathrm{n}}$ & $(1,1,0, \ldots, 0,0)$ & $\frac{1}{3}(2 n-1)(2 n+1)(2 n+3)$ & $n \geq 3$ \\
\hline $\mathrm{B}_{\mathrm{n}}$ & $(3,0,0, \ldots, 0,0)$ & $\frac{1}{3} n(2 n+1)(2 n+5)$ & $n \geq 2$ \\
\hline $\mathrm{B}_{\mathrm{n}}$ & $(0,2,0, \ldots, 0,0)$ & $\frac{1}{3}(n-1)(n+1)(2 n+1)(2 n+3)$ & $n \geq 3$ \\
\hline $\mathrm{C}_{\mathrm{n}}$ & $(1,1,0, \ldots, 0,0)$ & $\frac{2}{3} n(2 n-2)(2 n+2)$ & $n \geq 3$ \\
\hline $\mathrm{C}_{\mathrm{n}}$ & $(3,0,0, \ldots, 0,0)$ & $\frac{2}{3} n(n+1)(2 n+1)$ & $n \geq 2$ \\
\hline $\mathrm{C}_{\mathrm{n}}$ & $(0,2,0, \ldots, 0,0)$ & $\frac{1}{3}(n-1) n(2 n-1)(2 n+3)$ & $n \geq 3$ \\
\hline $\mathrm{D}_{\mathrm{n}}$ & $(1,1,0, \ldots, 0,0)$ & $\frac{8}{3}(n-1) n(n+1)$ & $n \geq 4$ \\
\hline $\mathrm{D}_{\mathrm{n}}$ & $(3,0,0, \ldots, 0,0)$ & $\frac{2}{3} n(n+2)(2 n-1)$ & $n \geq 4$ \\
\hline $\mathrm{D}_{\mathrm{n}}$ & $(0,2,0, \ldots, 0,0)$ & $\frac{1}{3} n(2 n+1)(2 n-3)(n+1)$ & $n \geq 4$ \\
\hline
\end{tabular}

Proof. Weyl's dimension formula.

The next lemma gives a complete classification of the irreducible simple subgroups of the classical groups which satisfy the necessary condition for hyperpolar actions in Lemma 2.3 It turns out that, with a finite number of exceptions, these subgroups come from isotropy representations of symmetric spaces.

Lemma 2.6. Let $H$ be a compact simple Lie group and $\varrho$ an irreducible representation of $H$ satisfying the following condition:

$$
\begin{aligned}
2 \operatorname{dim} H \geq \operatorname{deg} \varrho-2 & \text { if } \varrho \text { is of real type, } \\
\operatorname{dim} H \geq \operatorname{deg} \varrho-1 & \text { if } \varrho \text { is of complex type, } \\
\operatorname{dim} H \geq \frac{3}{2} \operatorname{deg} \varrho-4 & \text { if } \varrho \text { is of quaternionic type, }
\end{aligned}
$$

i.e. the corresponding condition of Lemma 2.3. Then one of the following is true: 
i) $\varrho$ is of real type and equivalent to the isotropy representation of a symmetric space.

ii) $\varrho$ is of complex type and $\varrho \otimes \theta$ is equivalent to the isotropy representation of a Hermitian symmetric space, where $\theta$ is a one-dimensional representation of $\mathrm{U}(1)$.

iii) $\varrho$ is of quaternionic type and $\varrho \otimes \phi$ is equivalent to the isotropy representation of a Quaternion-Kähler symmetric space, where $\phi$ is the standard representation of $\mathrm{Sp}(1) ;$ see $\mathrm{BESSE}$, $p .408$.

iv) $\varrho$ is a representation from the table below:

\begin{tabular}{|c|c|r|r|r|c|}
\hline$H$ & $\varrho$ & $\operatorname{deg}(\varrho)$ & $\varepsilon(\varrho)$ & $\operatorname{dim}(H)$ & Description \\
\hline \hline $\mathrm{A}_{1}$ & $(6)$ & 7 & +1 & 3 & $\mathrm{~S}^{6} \varrho_{1}$ \\
\hline $\mathrm{A}_{6}$ & $\varrho_{3}$ & 35 & 0 & 48 & $\Lambda^{3} \varrho_{1}$ \\
\hline $\mathrm{A}_{7}$ & $\varrho_{3}$ & 56 & 0 & 63 & $\Lambda^{3} \varrho_{1}$ \\
\hline $\mathrm{B}_{3}$ & $\varrho_{3}$ & 8 & +1 & 21 & $\operatorname{Spin}(7)$ \\
\hline $\mathrm{B}_{3}$ & $(0,0,2)$ & 35 & +1 & 21 & \\
\hline $\mathrm{B}_{5}$ & $\varrho_{5}$ & 32 & -1 & 55 & spin \\
\hline $\mathrm{B}_{7}$ & $\varrho_{7}$ & 128 & +1 & 105 & spin \\
\hline $\mathrm{B}_{8}$ & $\varrho_{8}$ & 256 & +1 & 136 & spin \\
\hline $\mathrm{D}_{4}$ & $(1,0,1,0)$ & 56 & +1 & 28 & \\
\hline $\mathrm{D}_{7}$ & $\varrho_{6}, \varrho_{7}$ & 64 & 0 & 91 & half-spin \\
\hline $\mathrm{G}_{2}$ & $\varrho_{1}$ & 7 & +1 & 14 & Aut(Ca) \\
\hline $\mathrm{G}_{2}$ & $(2,0)$ & 27 & +1 & 14 & \\
\hline
\end{tabular}

Proof. The proof proceeds case-by-case, using the strong monotonicity of the map

$$
\left(c_{1}, \ldots, c_{n}\right) \mapsto \operatorname{deg}\left(\varrho_{\left(c_{1}, \ldots, c_{n}\right)}\right) ;
$$

see Lemma 3.1 of [ON1], and Weyl's dimension formula. See the Appendix for lists of low degree representations.

Using Lemma 2.3 many subgroups of the simple Lie groups can be excluded as candidates for hyperpolar actions. However, in the case of a classical Lie group, there still remains a number of subgroups which fulfill the condition in Lemma 2.3 But if one starts to consider pairs of such subgroups $(H, K)$, one observes that condition (2.4) is violated in most cases. Since it would be cumbersome to check condition (2.4) for all suitable combinations of maximal subgroups $H$ and $K$, we use another bound for $\operatorname{dim} H$ and $\operatorname{dim} K$. The idea here is that the dimension of principal orbits of a hyperpolar action on $G$ is greater or equal to $\operatorname{dim}(G)-\operatorname{rk}(\mathrm{G})$, thus if we have two subgroups $H$ and $K$ such that $\operatorname{dim}(H)$ and $\operatorname{dim}(K)$ are both less than $\frac{1}{2}(\operatorname{dim}(G)-\operatorname{rk}(\mathrm{G}))$, then the action cannot be hyperpolar. In addition, a number of other cases can be ruled out by the following conditions.

Lemma 2.7. $\quad$ i) Let $H, K \subseteq \mathrm{SO}(\mathrm{n})$ be closed subgroups, such that

$$
\operatorname{dim} H, \operatorname{dim} K<\frac{n^{2}}{4}-\frac{3}{4} n .
$$

Then the $H \times K$-action on $\mathrm{SO}(\mathrm{n})$ is not hyperpolar.

If $n$ is even, the $H$-action on $\mathrm{SO}(\mathrm{n}) / \mathrm{U}\left(\frac{\mathrm{n}}{2}\right)$ is not hyperpolar. 
ii) Let $H, K \subseteq \mathrm{SU}(\mathrm{n})$ be closed subgroups, such that

$$
\operatorname{dim} H, \operatorname{dim} K<\frac{n^{2}}{2}-n
$$

Then the $H \times K$-action on $\mathrm{SU}(\mathrm{n})$ is not hyperpolar.

The $H$-action on $\mathrm{SU}(\mathrm{n}) / \mathrm{SO}(\mathrm{n})$ is not hyperpolar.

If $n$ is even, the $H$-action on $\mathrm{SU}(\mathrm{n}) / \mathrm{Sp}\left(\frac{\mathrm{n}}{2}\right)$ is not hyperpolar.

iii) Let $H, K \subseteq \mathrm{Sp}(\mathrm{n})$ be closed subgroups, such that

$$
\operatorname{dim} H, \operatorname{dim} K<n^{2} .
$$

Then the $H \times K$-action on $\mathrm{Sp}(\mathrm{n})$ is not hyperpolar.

The $H$-action on $\mathrm{Sp}(\mathrm{n}) / \mathrm{U}(\mathrm{n})$ is not hyperpolar.

Proof. One checks easily that the inequalities (2.12), (2.13), (2.14) imply in all cases that the respective actions cannot be hyperpolar by (2.3).

Now we will apply the dimension conditions proved in the preceding lemma to the maximal connected subgroups of the classical groups. It turns out that the inequalities (2.12), (2.13) and (2.14) hold for almost all subgroups of the classical groups with the obvious exception of those subgroups $H \subset G$ such that $H \times H$ is well-known to act hyperpolarly on $G$; see [HPTT2.

Lemma 2.8. i) For the following subalgebras $\varrho(\mathfrak{h})$ of $\mathfrak{s o}(n)$ we have $\operatorname{dim} \mathfrak{h}<$ $\frac{n^{2}}{4}-\frac{3}{4} n$ (i.e. inequality (2.12) holds):

\begin{tabular}{|c|c|c|c|}
\hline $\mathfrak{h}$ & $\varrho$ & $n=\operatorname{deg} \varrho$ & Range \\
\hline \hline $\mathfrak{s o}(p)+\mathfrak{s} \mathfrak{c}(q)$ & $\varrho_{1} \otimes \varrho_{1}$ & $p q$ & $p, q \geq 3$ \\
\hline $\mathfrak{s p}(p)+\mathfrak{s} \mathfrak{p}(q)$ & $\varrho_{1} \otimes \varrho_{1}$ & $4 p q$ & $p \geq 1, q \geq 2, p q \neq 2$ \\
\hline $\mathfrak{h}$ simple & adjoint & $\operatorname{dim} \mathfrak{h}$ & $\operatorname{dim} \mathfrak{h} \geq 8$ \\
\hline $\mathfrak{s p}(k)$ & $P^{2}$ & $\left(\begin{array}{c}2 k \\
2\end{array}\right)-1$ & $k \geq 3$ \\
\hline $\mathfrak{s o}(k)$ & $\mathrm{S}^{2} \varrho_{1}-\mathrm{N}$ & $\frac{1}{2}(k-1)(k+2)$ & $k \geq 4$ \\
\hline $\mathfrak{s p}(4)$ & $\varrho_{4}$ & 42 & \\
\hline $\mathfrak{f}_{4}$ & $\varrho_{1}$ & 26 & \\
\hline $\mathfrak{s u}(8)$ & $\varrho_{4}$ & 70 & \\
\hline $\mathfrak{s o}(16)$ & $\varrho_{7}, \varrho_{8}$ & 128 & \\
\hline $\mathfrak{s o}(9)$ & $\varrho_{4}$ & 16 & \\
\hline $\mathfrak{a}_{1}$ & $(6)$ & 7 & \\
\hline $\mathfrak{b}_{3}$ & $(0,0,2)$ & 35 & \\
\hline $\mathfrak{b}_{7}$ & $\varrho_{7}$ & 128 & \\
\hline $\mathfrak{b}_{8}$ & $\varrho_{8}$ & 256 & \\
\hline $\mathfrak{d}_{4}$ & $(1,0,1,0)$ & 56 & \\
\hline $\mathfrak{g}_{2}$ & $(2,0)$ & 27 & \\
\hline
\end{tabular}

ii) For the following subalgebras $\varrho(\mathfrak{h})$ of $\mathfrak{s u}(n)$ we have $\operatorname{dim} \mathfrak{h}<\frac{n^{2}}{2}-n$ (i.e. inequality (2.13) holds): 


\begin{tabular}{|c|c|c|c|}
\hline $\mathfrak{h}$ & $\varrho$ & $n=\operatorname{deg} \varrho$ & Range \\
\hline \hline $\mathfrak{s u}(p)+\mathfrak{s u}(q)$ & $\varrho_{1} \otimes \varrho_{1}$ & $p q$ & $p \geq 3, q \geq 2$ \\
\hline $\mathfrak{s u}(k)$ & $\varrho_{2}$ & $\frac{1}{2} k(k-1)$ & $k \geq 5$ \\
\hline $\mathfrak{s u}(k)$ & $\mathrm{S}^{2} \varrho_{1}$ & $\frac{1}{2} k(k+1)$ & $k \geq 3$ \\
\hline $\mathfrak{s o}(10)$ & $\varrho_{4}, \varrho_{5}$ & 16 & \\
\hline $\mathfrak{e}_{6}$ & $\varrho_{1}$ & 27 & \\
\hline $\mathfrak{s u}(7)$ & $\varrho_{3}$ & 35 & \\
\hline $\mathfrak{s u}(8)$ & $\varrho_{3}$ & 56 & \\
\hline $\mathfrak{d}_{7}$ & $\varrho_{6}, \varrho_{7}$ & 64 & \\
\hline \multicolumn{4}{|l}{} \\
\hline
\end{tabular}

iii) For the following subalgebras $\varrho(\mathfrak{h})$ of $\mathfrak{s p}(n)$ we have $\operatorname{dim} \mathfrak{h}<n^{2}$ (i.e. inequality (2.14) holds):

\begin{tabular}{|c|c|c|c|}
\hline $\mathfrak{h}$ & $\varrho$ & $2 n=\operatorname{deg} \varrho$ & Range \\
\hline \hline $\mathfrak{s o}(p)+\mathfrak{s p}(q)$ & $\varrho_{1} \otimes \varrho_{1}$ & $2 p q$ & $p \geq 3, q \geq 1$ \\
\hline $\mathfrak{s u}(6)$ & $\varrho_{3}$ & 20 & \\
\hline $\mathfrak{s o}(12)$ & $\varrho_{5}, \varrho_{6}$ & 32 & \\
\hline $\mathfrak{e}_{7}$ & $\varrho_{1}$ & 56 & \\
\hline $\mathfrak{s p}(3)$ & $\varrho_{3}$ & 14 & \\
\hline $\mathfrak{s u}(2)$ & $\mathrm{S}^{3} \varrho_{1}$ & 4 & \\
\hline $\mathfrak{b}_{5}$ & $\varrho_{5}$ & 32 & \\
\hline \multicolumn{4}{|r}{} \\
\hline
\end{tabular}

iv) In particular, the only maximal connected subgroups of the classical Lie groups $\mathrm{SO}(\mathrm{n})(n \geq 7), \mathrm{SU}(\mathrm{n})(n \geq 2)$ and $\mathrm{Sp}(\mathrm{n})(n \geq 2)$ for which the respective inequality (2.12), (2.13) or 2.14) does not hold are the connected symmetric subgroups and $\mathrm{G}_{2} \subset \mathrm{SO}(7)$.

Proof. For parts i), ii) and iii) of the lemma, one has to verify case-by-case that the dimensions of the Lie algebra $\mathfrak{h}$ and the degree of its representation $\varrho$ fulfill the respective inequalities. In some cases, a simple induction proof may be used.

Now let us prove part iv) of the lemma. For a symmetric subgroup $H \subset G$ it is clear that the respective inequality (2.12), (2.13) or (2.14) does not hold, because we have the Hermann action of $H \times H$ on $G$ which is hyperpolar. Similarly, $\mathrm{G}_{2} \times \mathrm{G}_{2}$ acts hyperpolarly on $\mathrm{SO}(7)$; see [HPTT2].

On the other hand, consider the subgroups of the classical Lie groups given in Theorem 2.2, Let us start with the maximal connected subgroups of $\mathrm{SO}(\mathrm{n})$ : The subgroups $\mathrm{SO}(\mathrm{k}) \times \mathrm{SO}(\mathrm{n}-\mathrm{k}) \subset \mathrm{SO}(\mathrm{n})$ and $\mathrm{U}(\mathrm{k}) \subset \mathrm{SO}(2 \mathrm{k})$ are symmetric. The subgroups of the form $\mathrm{SO}(\mathrm{p}) \otimes \mathrm{SO}(\mathrm{q})$ and $\mathrm{Sp}(\mathrm{p}) \otimes \mathrm{Sp}(\mathrm{q})$ are excluded by part i) of this lemma, with the exception of $\operatorname{Sp}(1) \otimes \mathrm{Sp}(2)$, which is a symmetric subgroup of $\mathrm{SO}(8)$. It remains to consider the subgroups of the form $\varrho(H) \subset \mathrm{SO}(\mathrm{n})$, where $H$ is a simple Lie group and $\varrho$ is an irreducible real representation of $H$. We only have to consider the representations $\varrho$ given in Lemma 2.6, because if condition (2.6) is violated, then (2.12) holds. The only subgroups not excluded by part i) are $\mathrm{G}_{2} \subset \mathrm{SO}(7)$ and the symmetric subgroup $\operatorname{Spin}(7) \subset \mathrm{SO}(8)$.

To prove part iv) for subgroups of $\mathrm{SU}(\mathrm{n})$ and $\mathrm{Sp}(\mathrm{n})$, one proceeds in an analogous manner.

Part iv) of this lemma is an important observation for our classification, because it shows that essentially only such maximal connected non-transitive subgroups 
$H \times K \subset G \times G$ have to be considered where $K \subset G$ is a symmetric subgroup. Thus, the number of individual cases to be examined is reduced considerably and our classification problem is essentially reduced to the classification of the hyperpolar actions on the compact symmetric spaces of type III, i.e. on the spaces $G / K$ where $G$ is a simple compact Lie group and $K$ a symmetric subgroup.

2.3. Slice Representations. Having excluded many candidates for hyperpolar actions by dimension conditions, we will now take a closer look at some of the remaining actions. We will compute a slice representation for each of these actions in order to decide whether they are polar or to determine the cohomogeneity using Theorem 1.7 In all cases, the identity element of $G$ happens to be contained in a singular orbit, therefore the slice representation at $e$ is non-trivial in all cases.

Let $H, K \subset G$. Consider the $H \times K$-action on $G$. The slice representation at $e \in G$ may be computed as follows: The isotropy group of $H \times K$ at $e$ is

$$
(H \times K)_{e}=\{(h, h) \mid h \in H \cap K\}=\Delta(H \cap K) .
$$

The tangent space of the orbit of $e$ at $e$ is

$$
\mathrm{T}_{\mathrm{e}}(\mathrm{H} \times \mathrm{K}) \mathrm{e}=\mathfrak{h}+\mathfrak{k} .
$$

Consequently, the normal space at $e$ equals

$$
\nu_{e}(H \times K) e=\mathfrak{h}^{\perp} \cap \mathfrak{k}^{\perp},
$$

where $\mathfrak{h}^{\perp}$ and $\mathfrak{k}^{\perp}$ are the orthogonal complements of $\mathfrak{h}$ and $\mathfrak{k}$ with respect to the negative of the Killing form. The isotropy representation at $e$ is equivalent to the adjoint representation of $G$, restricted to $H \cap K$. Hence the slice representation is equivalent to the adjoint representation of $G$, restricted to $H \cap K$, on the invariant subspace $\mathfrak{h}^{\perp} \cap \mathfrak{k}^{\perp}$.

In the case of the $H$-action on $G / K$, the isotropy group at $e K$ is $H_{e K}=H \cap K$, hence isomorphic to (2.15), and the slice representation at $e K$ is equivalent to that of the $H \times K$-action on $G$ at $e$. Thus, in order to compute slice representations, it is not necessary to distinguish between the $H \times K$-action on $G$ and the $H$-action on $G / K$. In particular, the cohomogeneities of the two actions are equal.

We will frequently use the following lemma to show that certain slice representations are not polar.

Lemma 2.9. Let $G$ be a compact connected Lie group and $\varrho: G \rightarrow \mathrm{SO}(\mathrm{n})$ be a representation. If the representation module $\mathbb{R}^{n}$ contains two invariant subspaces that are non-trivial equivalent representation modules of $G$, then $\varrho$ is not polar.

Proof. Assume $\varrho: G \rightarrow \mathrm{SO}(\mathrm{n})$ is a polar representation. Let $\mathbb{R}^{n}=V_{1} \oplus V_{2} \oplus W$ be a $G$-invariant decomposition, where $V_{i}$ are non-trivial irreducible equivalent $G$ modules. By DADOK, Theorem 4, we have that the actions of $G$ on $V_{i}$ and on $V_{1} \oplus V_{2}$ are polar, a section of the $G$-action on $V_{1} \oplus V_{2}$ is of the form $a=a_{1} \oplus a_{2}$, where $a_{i} \subset V_{i}$ are sections, and the action $H_{1} \times H_{2}$ on $V_{1} \oplus V_{2}$ by components has the same orbits as the $G$-action on $V_{1} \oplus V_{2}$, where $H_{i}$ are the connected subgroups of $G$ corresponding to $\mathfrak{h}_{1}=Z\left(a_{2}\right)$ (the centralizer of $a_{2}$ in $\mathfrak{g}$ ) and $\mathfrak{h}_{2}=Z\left(a_{1}\right)$, respectively. But this is a contradiction since the groups $H_{i}$ act reducibly on $V_{i}$.

Notation. In the following, we define the group $\operatorname{Sp}(\mathrm{n})$ as the group of norm-preserving automorphisms of $\mathbb{H}^{n}$ and thus as a subgroup of $\mathrm{G} \ell(\mathrm{n}, \mathbb{H})$. The Lie algebra of $\mathrm{Sp}(\mathrm{n})$ is given as the set of skew-hermitian quaternionic $n \times n$-matrices. We denote 
by $H_{1} \otimes H_{2}$ the Kronecker product of two matrix Lie groups. We use the following notation for various representation modules: $\mathbb{R}^{\mu}, \mathbb{C}^{\mu}, \mathbb{H}^{\mu}$ denotes the standard representation space of $\mathrm{O}(\mu), \mathrm{U}(\mu)$ or $\operatorname{Sp}(\mu)$, respectively. By $\mathbb{R}^{\mu \times \nu}, \mathbb{C}^{\mu \times \nu}, \mathbb{H}^{\mu \times \nu}$ we denote the space of $\mu \times \nu$-matrices as representation spaces of $\mathrm{O}(\mu) \times \mathrm{O}(\nu)$, $\mathrm{U}(\mu) \times \mathrm{U}(\nu)$ or $\mathrm{O}(\mu) \times \mathrm{Sp}(\nu)$, respectively, where the groups act by the tensor product of the standard representations. We denote by $\mathbb{R}_{0}^{\mu \times \mu}$ or $\mathbb{C}_{0}^{\mu \times \mu}$ the space of real or complex $\mu \times \mu$-matrices with zero trace as representation spaces of $\mathrm{O}(\mu)$ or $\mathrm{U}(\mu)$, respectively, where the groups act by conjugation. We assume that subgroups of the classical groups are standardly embedded, e.g. by $\mathrm{SO}(\mathrm{m}) \times \mathrm{SO}(\mathrm{n})$ we denote the subgroup

$$
\left\{\left(\begin{array}{l|l}
A & \\
\hline & B
\end{array}\right) \mid A \in \mathrm{SO}(\mathrm{m}), \mathrm{B} \in \mathrm{SO}(\mathrm{n})\right\} \subset \mathrm{SO}(\mathrm{m}+\mathrm{n}) .
$$

2.3.1. Actions of $\mathrm{SO}(p) \otimes \mathrm{SO}(q), \mathrm{SU}(p) \otimes \mathrm{SU}(q)$ and $\mathrm{SO}(p) \otimes \mathrm{Sp}(q)$. We study the following actions of $H$ on $G / K$, where $n$ ranges from 1 to $\left\lfloor\frac{p q}{2}\right\rfloor$ :

(a) $H=\mathrm{SO}(\mathrm{p}) \otimes \mathrm{SO}(\mathrm{q}), \quad p \geq 3, q \geq 3, \quad G / K=\mathrm{SO}(\mathrm{pq}) / \mathrm{SO}(\mathrm{n}) \times \mathrm{SO}(\mathrm{pq}-\mathrm{n})$,

(b) $H=\mathrm{SU}(\mathrm{p}) \otimes \mathrm{SU}(\mathrm{q}), \quad p \geq 3, q \geq 2, \quad G / K=\mathrm{SU}(\mathrm{pq}) / \mathrm{S}\left(\mathrm{U}_{\mathrm{n}} \times \mathrm{U}_{\mathrm{pq}-\mathrm{n}}\right)$,

(c) $H=\mathrm{SO}(\mathrm{p}) \otimes \mathrm{Sp}(\mathrm{q}), \quad p \geq 3, q \geq 1, \quad G / K=\mathrm{Sp}(\mathrm{pq}) / \mathrm{Sp}(\mathrm{n}) \times \mathrm{Sp}(\mathrm{pq}-\mathrm{n})$.

We will treat these three types of actions simultaneously.

Case 1. We start with the special case where $n$ is a multiple of $q$, i.e.

$$
n=\ell q, \quad \ell \in\left\{1, \ldots,\left\lfloor\frac{p}{2}\right\rfloor\right\}
$$

In this case it is easy to determine the group $H \cap K$. The condition $A \otimes B \in K$ implies that $a_{\mu \nu} B=a_{\nu \mu} B=0$ if $\mu>\ell$ and $\nu \leq \ell$, i.e.

$$
A \otimes B=\left(\begin{array}{cccccc}
a_{11} B & \ldots & a_{1 \ell} B & 0 & \ldots & 0 \\
\vdots & & \vdots & \vdots & & \vdots \\
a_{\ell 1} B & \ldots & a_{\ell \ell} B & 0 & \ldots & 0 \\
0 & \ldots & 0 & a_{\ell+1, \ell+1} B & \ldots & a_{\ell+1, p q} B \\
\vdots & & \vdots & \vdots & & \vdots \\
0 & \ldots & 0 & a_{p q, \ell+1} B & \ldots & a_{p q, p q} B
\end{array}\right)
$$

Since the matrices $B$ are invertible it follows that:

$$
\begin{aligned}
& \text { (a) } H \cap K=[\mathrm{S}(\mathrm{O}(\ell) \times \mathrm{O}(\mathrm{p}-\ell))] \otimes \mathrm{SO}(\mathrm{q}) \text {, } \\
& \text { (b) } H \cap K=[\mathrm{S}(\mathrm{U}(\ell) \times \mathrm{U}(\mathrm{p}-\ell))] \otimes \mathrm{SU}(\mathrm{q}), \\
& \text { (c) } H \cap K=[\mathrm{S}(\mathrm{O}(\ell) \times \mathrm{O}(\mathrm{p}-\ell))] \otimes \mathrm{Sp}(\mathrm{q})
\end{aligned}
$$

The slice representation is equivalent to the action of the adjoint representation of $G$, restricted to $H \cap K$, on the invariant subspace $\mathfrak{h}^{\perp} \cap \mathfrak{k}^{\perp}$. In order to determine $\mathfrak{h}^{\perp} \cap \mathfrak{k}^{\perp}$, we first compute the subalgebras $\mathfrak{h}$. One obtains the following matrix Lie 
algebras, where $I_{q}$ denotes the $q \times q$ identity matrix:
(a) $\left\{\left(\begin{array}{ccc}x_{11} I_{q} & \ldots & x_{1 p} I_{q} \\ \vdots & & \vdots \\ x_{p 1} I_{q} & \ldots & x_{p p} I_{q}\end{array}\right) \mid \begin{array}{c}x_{i j} \in \mathbb{R} \\ x_{i j}=-x_{j i}\end{array}\right\}+\left\{\left(\begin{array}{ccc}Y & & \\ & \ddots & \\ & & Y\end{array}\right) \mid Y \in \mathfrak{s o}(q)\right\}$,
(b) $\left\{\left(\begin{array}{ccc}x_{11} I_{q} & \ldots & x_{1 p} I_{q} \\ \vdots & & \vdots \\ x_{p 1} I_{q} & \ldots & x_{p p} I_{q}\end{array}\right) \mid \begin{array}{c}x_{i j} \in \mathbb{C} \\ x_{i j}=-\bar{x}_{j i}\end{array}\right\}+\left\{\left(\begin{array}{ccc}Y & & \\ & \ddots & \\ & & Y\end{array}\right) \mid Y \in \mathfrak{s u}(q)\right\}$,
(c) $\left\{\left(\begin{array}{ccc}x_{11} I_{q} & \ldots & x_{1 p} I_{q} \\ \vdots & & \vdots \\ x_{p 1} I_{q} & \ldots & x_{p p} I_{q}\end{array}\right) \mid \begin{array}{c}x_{i j} \in \mathbb{R} \\ x_{i j}=-x_{j i}\end{array}\right\}+\left\{\left(\begin{array}{ccc}Y & & \\ & \ddots & \\ & & Y\end{array}\right) \mid Y \in \mathfrak{s p}(q)\right\}$.

Their orthogonal complements in $\mathfrak{s o}(p q), \mathfrak{s u}(p q), \mathfrak{s p}(p q)$, respectively, are:
(a) $\mathfrak{h}^{\perp}=\left\{\left(\begin{array}{ccc}X_{11} & \ldots & X_{1 p} \\ \vdots & & \vdots \\ X_{p 1} & \ldots & X_{p p}\end{array}\right) \mid \begin{array}{cc}X_{i j} \in \mathbb{R}^{q \times q} & \operatorname{tr} X_{\mathrm{ij}}=0, \\ X_{i j}=-X_{j i}^{t}, & \sum_{i=1}^{p} X_{i i}=0\end{array}\right\}$,
(b) $\mathfrak{h}^{\perp}=\left\{\left(\begin{array}{ccc}X_{11} & \ldots & X_{1 p} \\ \vdots & & \vdots \\ X_{p 1} & \ldots & X_{p p}\end{array}\right) \mid \begin{array}{cc}X_{i j} \in \mathbb{C}^{q \times q}, & \operatorname{tr} X_{\mathrm{ij}}=0, \\ X_{i j}=-\bar{X}_{j i}^{t}, & \sum_{i=1}^{p} X_{i i}=0\end{array}\right\}$,
(c) $\mathfrak{h}^{\perp}=\left\{\left(\begin{array}{ccc}X_{11} & \ldots & X_{1 p} \\ \vdots & & \vdots \\ X_{p 1} & \ldots & X_{p p}\end{array}\right) \mid \begin{array}{cc}X_{i j} \in \mathbb{H}^{q \times q}, & \Re\left(\operatorname{tr} X_{\mathrm{ij}}\right)=0, \\ X_{i j}=-\bar{X}_{j i}^{t}, & \sum_{i=1}^{p} X_{i i}=0\end{array}\right\}$.

Finally, we obtain the normal space at the identity element by intersection with $\mathfrak{k}^{\perp}$ :

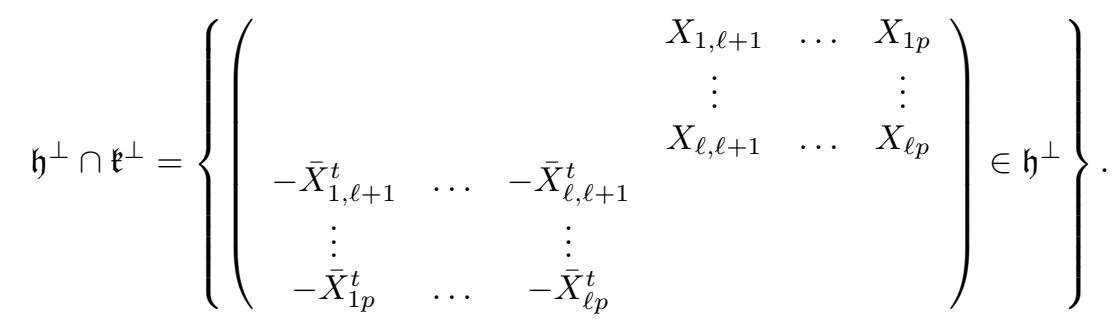

From the above formulae one can determine the action of $H \cap K$ on (2.20).

(a) It is easy to see that the $H \cap K$-module (2.20) is equivalent to $\mathbb{R}^{\ell} \otimes \mathbb{R}^{p-\ell} \otimes$ $\mathbb{R}_{0}^{q \times q}$, where the third factor denotes the traceless real $q \times q$-matrices. The action of $H \cap K=[\mathrm{S}(\mathrm{O}(\ell) \times \mathrm{O}(\mathrm{p}-\ell))] \otimes \mathrm{SO}(\mathrm{q})$ is given by the standard representations of $\mathrm{O}(\ell)$ and $\mathrm{O}(\mathrm{p}-\ell)$ on the first two factors and by conjugation with $\mathrm{SO}(\mathrm{q})$ on the third. By [DADOK], none of these representations is polar for $p, q \geq 3$.

(b) Here we have the action of $H \cap K=[\mathrm{S}(\mathrm{U}(\ell) \times \mathrm{U}(\mathrm{p}-\ell))] \otimes \mathrm{SU}(\mathrm{q})$ on $\mathbb{C}^{\ell} \otimes$ $\mathbb{C}^{p-\ell} \otimes \mathbb{C}_{0}^{q \times q}$, which is not polar; see [DADOK].

(c) In this case $(2.20)$ is equivalent to $\mathbb{R}^{\ell} \otimes \mathbb{R}^{p-\ell} \otimes U$, where $U$ denotes the quaternionic $q \times q$-matrices with imaginary trace. The $\operatorname{Sp}(\mathrm{q})$-module $U$ contains the set of skew-hermitian quaternionic matrices $\mathfrak{s p}(q)$ as an invariant subspace, thus the action of $\operatorname{Sp}(q)$ by conjugation on this subspace is equivalent to the adjoint representation. If $q=1$, then $U=\Im(\mathbb{H})$ and the action of $\mathrm{Sp}(1)$ is equivalent to the standard representation of $\mathrm{SO}(3)$; then the slice representation is polar only 
if $\ell=1$. But in this case $n=1$ and thus $\operatorname{rk}(\mathrm{G} / \mathrm{K})=1$; on the other hand, $p \geq 3$ and therefore the cohomogeneity of the action is at least two. (The slice representation is equivalent to the isotropy representation of the symmetric space $\mathrm{SO}(\mathrm{p}+2) / \mathrm{SO}(3) \times \mathrm{SO}(\mathrm{p}-1)$ in this case. $)$ Thus the corresponding action of $H$ on $G / K$ cannot be hyperpolar. If $q \geq 2$ or $\ell \in\left\{2, \ldots,\left\lfloor\frac{p}{2}\right\rfloor\right\}$ the slice representation is not polar by [DADOK].

Case 2. Consider the other special case where $n$ is not a multiple of $q$, i.e.

$$
n=\ell q+m, \quad \ell \in\left\{0, \ldots,\left\lfloor\frac{p-1}{2}\right\rfloor\right\}, m \in\{1, \ldots, q-1\} .
$$

Again, the group $H \cap K$ is easily determined:

(a) $H \cap K=[\mathrm{S}(\mathrm{O}(\ell) \times \mathrm{O}(1) \times \mathrm{O}(\mathrm{p}-\ell-1))] \otimes[\mathrm{S}(\mathrm{O}(\mathrm{m}) \times \mathrm{O}(\mathrm{q}-\mathrm{m}))]$,

(b) $H \cap K=[\mathrm{S}(\mathrm{U}(\ell) \times \mathrm{U}(1) \times \mathrm{U}(\mathrm{p}-\ell-1))] \otimes\left[\mathrm{S}\left(\mathrm{U}_{m} \times \mathrm{U}_{q-m}\right)\right]$,

(c) $H \cap K=[\mathrm{S}(\mathrm{O}(\ell) \times \mathrm{O}(1) \times \mathrm{O}(\mathrm{p}-\ell-1))] \otimes[\mathrm{Sp}(\mathrm{m}) \times \mathrm{Sp}(\mathrm{q}-\mathrm{m})]$.

We compute the normal space $\mathfrak{h}^{\perp} \cap \mathfrak{k}^{\perp}$ :

$$
\mathfrak{h}^{\perp} \cap \mathfrak{k}^{\perp}=\left\{\left(\begin{array}{rc}
0 & M \\
-\bar{M}^{t} & 0
\end{array}\right) \in \mathfrak{h}^{\perp}\right\}
$$

where $M$ is the $n \times(p q-n)$-matrix defined as follows:

$$
M=\left(\begin{array}{llllll}
B_{1, \ell+1} & A_{1, \ell+2} & B_{1, \ell+2} & \ldots & A_{1 p} & B_{1 p} \\
D_{1, \ell+1} & C_{1, \ell+2} & D_{1, \ell+2} & \ldots & C_{1 p} & D_{1 p} \\
\vdots & \vdots & \vdots & & \vdots & \vdots \\
B_{\ell, \ell+1} & A_{\ell, \ell+2} & B_{\ell, \ell+2} & \ldots & A_{\ell p} & B_{\ell p} \\
D_{\ell, \ell+1} & C_{\ell, \ell+2} & D_{\ell, \ell+2} & \ldots & C_{\ell p} & D_{\ell p} \\
0 & A_{\ell+1, \ell+2} & B_{\ell+1, \ell+2} & \ldots & A_{\ell+1, p} & B_{\ell+1, p}
\end{array}\right) .
$$

Here $A_{i j}$ and $D_{i j}$ are $m \times m$ and $(q-m) \times(q-m)$-matrices, respectively. We have to distinguish the two cases where $\ell$ is zero and where $\ell$ is non-zero.

Case 2.1. Assume $\ell \geq 1$. Condition (2.21) implies $p \geq 3$.

(a) In the case of the first action, the matrices $A_{i j}, B_{i j}, C_{i j}$ and $D_{i j}$ are real matrices such that $\operatorname{tr} \mathrm{A}+\operatorname{trD}=0$. From the form of the matrix (2.22), one can read off the action of $H \cap K$. The factor $\mathrm{S}(\mathrm{O}(\mathrm{m}) \times \mathrm{O}(\mathrm{q}-\mathrm{m}))$ acts on the $q \times q$-submatrices

$$
\left(\begin{array}{cc}
A_{i j} & B_{i j} \\
C_{i j} & D_{i j}
\end{array}\right)
$$

by conjugation, i.e. these submatrices correspond to the module

$$
W=\mathbb{R}_{0}^{m \times m} \oplus \mathbb{R}^{m \times(q-m)} \oplus \mathbb{R}^{(q-m) \times m} \oplus \mathbb{R}_{0}^{(q-m) \times(q-m)} \oplus \mathbb{R},
$$

where the first four summands correspond to the submatrices $A_{i j}, B_{i j}, C_{i j}$ and $D_{i j}$, respectively, and the summand $\mathbb{R}$ corresponds to $\operatorname{tr} \mathrm{A}=-\operatorname{trD}$. On the other hand, consider the module

$$
V=\mathbb{R}^{\ell} \otimes \mathbb{R}^{p-\ell-1}
$$

of $\mathrm{O}(\ell) \times \mathrm{O}(\mathrm{p}-\ell-1)$. The slice representation contains as a summand the $H \cap K$ module $V \otimes W$, as can be seen from (2.22) and (2.23). But the module $W$ contains two equivalent summands isomorphic to $\mathbb{R}^{m \times(q-m)}$ of the group $\mathrm{S}(\mathrm{O}(\mathrm{m}) \times \mathrm{O}(\mathrm{q}-\mathrm{m}))$, thus by Lemma 2.9 the action on $V \otimes W$ is not polar. 
(b) and (c) An analogous argument as in case (a) shows that the slice representation is not polar by Lemma 2.9 .

Case 2.2. Now we consider the case $\ell=0$, where the matrix $M$ has the form

$$
M=\left(\begin{array}{llllll}
0 & A_{12} & B_{12} & \ldots & A_{1 p} & B_{1 p}
\end{array}\right),
$$

where in case (a): $A_{1 j} \in \mathbb{R}_{0}^{m \times m}, B_{1 j} \in \mathbb{R}^{m \times(q-m)}$; in case (b): $A_{1 j} \in \mathbb{C}_{0}^{m \times m}$, $B_{1 j} \in \mathbb{C}^{m \times(q-m)}$ and in case (c): $A_{1 j} \in \mathbb{H}^{m \times m}, \Re\left(\operatorname{trA}_{1 \mathrm{j}}\right)=0, B_{1 j} \in \mathbb{H}^{m \times(q-m)}$.

(a) The slice representation is equivalent to

$$
\left(\mathbb{R}_{0}^{m \times m} \oplus \mathbb{R}^{m \times(q-m)}\right) \otimes \mathbb{R}^{p-1},
$$

This is a polar representation iff $m=1$, i.e. $n=1$; see [DADOK. But if $n=1$ we have $\operatorname{rk}(\mathrm{G} / \mathrm{K})=1$ and the $H$-action can only be hyperpolar if its cohomogeneity is one, which is not the case if $p, q \geq 3$, as we assumed.

(b) For the $\mathrm{SU}(\mathrm{p}) \otimes \mathrm{SU}(\mathrm{q})$-action on $\mathrm{SU}(\mathrm{pq}) / \mathrm{S}\left(\mathrm{U}_{\mathrm{n}} \times \mathrm{U}_{\mathrm{pq}-\mathrm{n}}\right)$, the slice representation is equivalent to the action of $\mathrm{S}\left(\mathrm{U}_{m} \times \mathrm{U}_{q-m}\right) \otimes \mathrm{S}\left(\mathrm{U}_{1} \times \mathrm{U}_{p-1}\right)$ on

$$
\left(\mathbb{C}_{0}^{m \times m} \oplus \mathbb{C}^{m \times(q-m)}\right) \otimes \mathbb{C}^{p-1} .
$$

By [DADOK], the action on (2.29) is polar iff $m=1$, i.e. $n=1$; again the corresponding $H$-action on $G / K$ can only be hyperpolar if its cohomogeneity is one; this is the case only if $q=2$.

(c) In the case of the $\mathrm{SO}(\mathrm{p}) \otimes \mathrm{Sp}(\mathrm{q})$-action on $\mathrm{Sp}(\mathrm{pq}) / \mathrm{Sp}(\mathrm{n}) \times \mathrm{Sp}(\mathrm{pq}-\mathrm{n})$, the slice representation is equivalent to the action of $\mathrm{SO}(\mathrm{p}-1) \otimes[\mathrm{Sp}(\mathrm{m}) \times \mathrm{Sp}(\mathrm{q}-\mathrm{m})]$ on

$$
\left(U \oplus \mathbb{H}^{m \times(q-m)}\right) \otimes \mathbb{R}^{p-1},
$$

where $U$ denotes the quaternionic $m \times m$-matrices with imaginary trace. In (2.30), the invariant subspace $\mathbb{H}^{m \times(q-m)} \otimes \mathbb{R}^{p-1}$ is a polar representation only if $m=1$ and $q=2$; see DADOK. Now from $m=1$ it follows that $\operatorname{rk}(\mathrm{G} / \mathrm{K})=1$, thus if the $H$-action on $G / K$ is hyperpolar, it must have cohomogeneity one; but (2.30) is reducible, so the cohomogeneity is at least two.

Result. The actions (a) and (c) are not hyperpolar. Action (b) is hyperpolar only in the case of the $\mathrm{SU}(\mathrm{p}) \otimes \mathrm{SU}(2)$-action on $\mathbb{C P}^{2 p-1}$.

2.3.2. Actions of $\operatorname{Sp}(p) \otimes \operatorname{Sp}(q)$. In the following we will determine for which $p, q$ and $n$ the actions of $H$ on $G / K$, where

$$
H=\mathrm{Sp}(\mathrm{p}) \otimes \mathrm{Sp}(\mathrm{q}), \quad p \geq 2, q \geq 1, \quad G / K=\mathrm{SO}(4 \mathrm{pq}) / \mathrm{SO}(\mathrm{n}) \times \mathrm{SO}(4 \mathrm{pq}-\mathrm{n})
$$

are hyperpolar. In order to describe the subgroup $H=\mathrm{Sp}(\mathrm{p}) \otimes \mathrm{Sp}(\mathrm{q}) \subset \mathrm{SO}(4 \mathrm{pq})$ explicitly, we use the isomorphism of real algebras

$$
\mathbb{H} \otimes_{\mathbb{R}} \mathbb{H} \cong \mathbb{R}(4),
$$

where $\mathbb{R}(4)$ denotes the algebra of real $4 \times 4$-matrices. This isomorphism may be defined as follows: $\left(\mathbb{H}=\mathbb{R}^{4}=\mathbb{R} \oplus \mathbb{R} i \oplus \mathbb{R} j \oplus \mathbb{R} k\right)$

$$
\begin{aligned}
\mathbb{H} \otimes_{\mathbb{R}} \mathbb{H} & \rightarrow \mathbb{R}(4) \\
u \otimes v & \mapsto(q \mapsto u q \bar{v}), \quad u, v, q \in \mathbb{H} .
\end{aligned}
$$


We will use this isomorphism to express real $4 \times 4$-matrices as elements of $\mathbb{H} \otimes_{\mathbb{R}} \mathbb{H}$, e.g. we write $u \otimes 1$ or $1 \otimes \bar{u}$ for the matrices in $\mathbb{R}(4)$ given as left or right multiplication by $u \in \mathbb{H}$. Using this convention, the subgroup $H$ may be written as follows:

$$
\begin{gathered}
H=\left\{\left(\begin{array}{ccc}
M_{11} & \ldots & M_{1 p} \\
\vdots & & \vdots \\
M_{p 1} & \ldots & M_{p p}
\end{array}\right) \mid M_{\mu \nu}=\left(\begin{array}{ccc}
a_{\mu \nu} \otimes b_{11} & \ldots & a_{\mu \nu} \otimes b_{1 q} \\
\vdots & & \vdots \\
a_{\mu \nu} \otimes b_{q 1} & \ldots & a_{\mu \nu} \otimes b_{q q}
\end{array}\right),\right. \\
\left.\left(\begin{array}{ccc}
a_{11} & \ldots & a_{1 p} \\
\vdots & & \vdots \\
a_{p 1} & \ldots & a_{p p}
\end{array}\right) \in \operatorname{Sp}(\mathrm{p}), \quad\left(\begin{array}{ccc}
b_{11} & \ldots & b_{1 q} \\
\vdots & & \vdots \\
b_{q 1} & \ldots & b_{q q}
\end{array}\right) \in \mathrm{Sp}(\mathrm{q})\right\} .
\end{gathered}
$$

From this, one gets the orthogonal complement of its Lie algebra

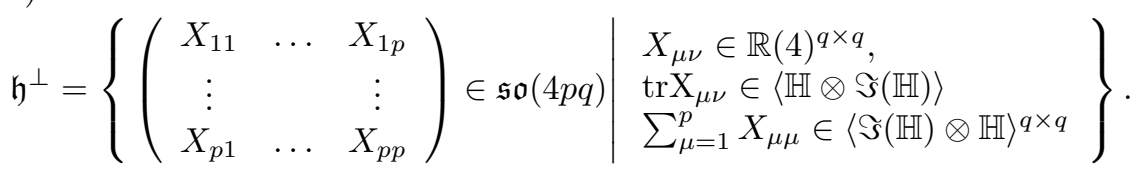

Note that in the formulae above the matrices $M_{\mu \nu}$ and $X_{\mu \nu}$ are considered as $q \times q$ matrices with entries from $\mathbb{H} \otimes_{\mathbb{R}} \mathbb{H}=\mathbb{R}(4)$. In particular, the trace of such a matrix is also an element of $\mathbb{R}(4)$. (Alternatively, they could be viewed as real $4 q \times 4 q$-matrices.) Here we used the abbreviation e.g.

$$
\langle\mathbb{H} \otimes \Im(\mathbb{H})\rangle=\operatorname{span}_{\mathbb{R}}\{\mathrm{u} \otimes \mathrm{v} \mid \mathrm{u} \in \mathbb{H}, \mathrm{v} \in \Im(\mathbb{H})\} .
$$

Case 1. We begin with the special case where $n$ is divisible by $4 q$, i.e.

$$
n=4 \ell q, \quad \ell \in\left\{1, \ldots,\left\lfloor\frac{p}{2}\right\rfloor\right\} .
$$

Then the isotropy group of $e K$ is

$$
H \cap K=[\operatorname{Sp}(\ell) \times \operatorname{Sp}(\mathrm{p}-\ell)] \otimes \operatorname{Sp}(\mathrm{q})
$$

and the normal space $\mathfrak{h}^{\perp} \cap \mathfrak{k}^{\perp}$ has the form (2.20), where in this case $\mathfrak{h}^{\perp}$ is given by (2.33). The $H \cap K$-module $\mathfrak{h}^{\perp} \cap \mathfrak{k}^{\perp}$ is isomorphic to the real vector space

$$
\mathbb{H}^{\ell \times(p-\ell)} \otimes_{\mathbb{R}} U,
$$

where $U$ denotes the quaternionic $q \times q$-matrices with imaginary trace. The group $H \cap K$ acts on (2.37) by the tensor product of the standard representations of $\operatorname{Sp}(\ell) \times \operatorname{Sp}(\mathrm{p}-\ell)$ on the first factor and by conjugation with matrices in $\operatorname{Sp}(\mathrm{q})$ on the second factor. By DADOK, the only such representation that is polar is the case $p=2, \ell=1, q=1$, which corresponds to the action of $H=\operatorname{Sp}(1) \otimes \operatorname{Sp}(2)$ on $G / K=\mathrm{SO}(8) / \mathrm{SO}(4) \times \mathrm{SO}(4)$. But here both subgroups are symmetric, so in this case we have a Hermann action.

Case 2. Now assume $n$ is a multiple of 4 , but not of $4 q$, i.e.

$$
n=4(\ell q+m), \quad \ell \in\left\{0, \ldots,\left\lfloor\frac{p-1}{2}\right\rfloor\right\}, \quad m \in\{1, \ldots, q-1\} .
$$

In particular, it follows that $q \geq 2$. Then from (2.32), one can read off the isotropy group at $e K$ to be

$$
H \cap K=[\operatorname{Sp}(\ell) \times \operatorname{Sp}(1) \times \operatorname{Sp}(\mathrm{p}-\ell-1)] \otimes[\operatorname{Sp}(\mathrm{m}) \times \mathrm{Sp}(\mathrm{q}-\mathrm{m})] .
$$


Case 2.1. If $\ell \geq 1$, then (2.38) implies $p \geq 3$. In this case the slice representation contains two equivalent summands and is not polar by Lemma 2.9] (cf. [2.3.1] Case 2.1).

Case 2.2. If $\ell=0$, then

$$
H \cap K=[\mathrm{Sp}(1) \times \mathrm{Sp}(\mathrm{p}-1)] \otimes[\mathrm{Sp}(\mathrm{m}) \times \mathrm{Sp}(\mathrm{q}-\mathrm{m})]
$$

acts on one summand of the slice representation by the tensor product of all four factors. Following [DADOK], the only such polar representation is $\mathrm{SO}(4) \otimes \mathrm{SO}(4)$, i.e. $p=2, m=1, q=2$, but the corresponding action of $H=\operatorname{Sp}(2) \otimes \operatorname{Sp}(2)$ on $G / K=\mathrm{SO}(16) / \mathrm{SO}(4) \times \mathrm{SO}(12)$ cannot be hyperpolar because $\operatorname{dim}(H)=20$ and $\operatorname{dim}(G / K)=48$, thus the cohomogeneity is at least 28 .

Case 3. Finally, we have to consider the case where $n$ is not divisible by 4 , i.e. assume

$$
\begin{gathered}
n=4(\ell q+m)+\kappa, \quad \ell \in\left\{0, \ldots,\left\lfloor\frac{p-1}{2}\right\rfloor\right\} \\
m \in\{0, \ldots, q-1\}, \kappa \in\{1,2,3\} .
\end{gathered}
$$

In this case, one obtains $H \cap K$ is equal to

$$
\left\{\left(\begin{array}{ccc}
P & & \\
& u & \\
& & Q
\end{array}\right) \otimes\left(\begin{array}{lll}
R & & \\
& v & \\
& & S
\end{array}\right) \mid \begin{array}{l}
P \in \mathrm{Sp}(\ell), \mathrm{R} \in \mathrm{Sp}(\mathrm{m}), \mathrm{u}, \mathrm{v} \in \mathrm{Sp}(1), \\
u \otimes v \in \mathrm{S}(\mathrm{O}(\kappa) \times \mathrm{O}(4-\kappa)) \\
Q \in \mathrm{Sp}(\mathrm{p}-\ell-1), \mathrm{S} \in \mathrm{Sp}(\mathrm{q}-\mathrm{m}-1)
\end{array}\right\} .
$$

Case 3.1. If $\ell \geq 1$, the slice representation is not polar by Lemma 2.9 (cf. Case 2.1 in 2.3.1).

Case 3.2. Assume $\ell=0$.

Case 3.2.1. Assume further $q=1$. It follows from (2.41) that $n=1,2$ or 3 .

$n=1$. It is well-known that the group $H=\operatorname{Sp}(\mathrm{p}) \otimes \operatorname{Sp}(1)$ acts transitively on the $(4 p-1)$-dimensional sphere $G / K=\mathrm{SO}(4 \mathrm{p}) / \mathrm{SO}(4 \mathrm{p}-1)$.

$n=2$. In this paragraph we will show that $H=\mathrm{Sp}(\mathrm{p}) \otimes \mathrm{Sp}(1) \subset \mathrm{SO}(4 \mathrm{p})$ acts on the Grassmannian $G / K=\mathrm{SO}(4 \mathrm{p}) / \mathrm{SO}(2) \times \mathrm{SO}(4 \mathrm{p}-2)$ with cohomogeneity one and thus hyperpolarly. We show this by considering the action of $H$ on the Stiefel manifold $\mathrm{V}_{2}\left(\mathbb{R}^{4 \mathrm{p}}\right)=\mathrm{SO}(4 \mathrm{p}) / \mathrm{SO}(4 \mathrm{p}-2)$ of orthonormal two-frames in $\mathbb{R}^{4 p}$. Consider first the action of $H$ on $\mathbb{R}^{4 p}$, which corresponds to the case $n=1$, i.e. the transitive action on the unit sphere. By (2.42), the isotropy group of the first canonical basis vector $e_{1} \in \mathbb{R}^{4 p}$ is

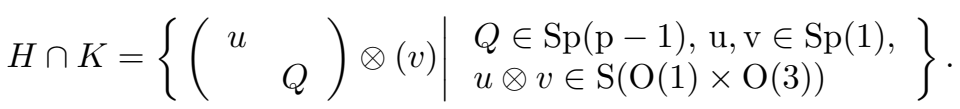

This group acts with cohomogeneity one on the unit sphere in $\left\{e_{1}\right\}^{\perp} \subset \mathbb{R}^{4 p}$, thus with cohomogeneity one on $\mathrm{V}_{2}\left(\mathbb{R}^{4 \mathrm{p}}\right)$. Now it follows that the action of $H$ on the Grassmannian $G / K=\mathrm{SO}(4 \mathrm{p}) / \mathrm{SO}(2) \times \mathrm{SO}(4 \mathrm{p}-2)$ must have cohomogeneity one or be transitive, but it is known that the action is non-transitive; see [N1]. Thus we have found a cohomogeneity one action that is not a Hermann action.

$n=3$. Here we have to consider the action of $H=\operatorname{Sp}(\mathrm{p}) \otimes \operatorname{Sp}(1)$ on $G / K=$ $\mathrm{SO}(4 \mathrm{p}) / \mathrm{SO}(3) \times \mathrm{SO}(4 \mathrm{p}-3)$. We will compute the slice representation at $e K$ in 
order to decide if this action is polar. We may assume $p \geq 3$ because for $p=2$ we have a Hermann action. From (2.42), we have

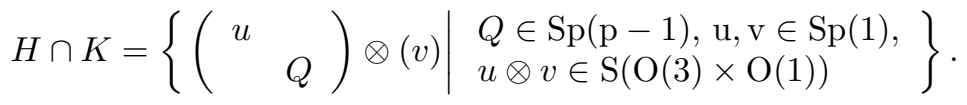

From the dimension of $H, G / K$ and $H \cap K$ we compute $\operatorname{dim}\left(\mathfrak{h}^{\perp} \cap \mathfrak{k}^{\perp}\right)=8(p-1)$. Now consider the group

$$
\left\{\begin{array}{ll}
\left(\begin{array}{ll}
A & \\
& B
\end{array}\right) \mid \begin{array}{l}
A \in \mathrm{S}(\mathrm{O}(3) \times \mathrm{O}(1)) \\
B \in \mathrm{Sp}(\mathrm{p}-1) \otimes \mathrm{Sp}(1)
\end{array}
\end{array}\right\} \subset \mathrm{SO}(4 \mathrm{p})
$$

which contains $H \cap K$. The group (2.45) acts on the space of matrices

$$
k^{\perp}=\left\{\left(\begin{array}{cc}
0 & X \\
-X^{t} & 0
\end{array}\right) \mid X \in \mathbb{R}^{3 \times(4 p-3)}\right\}
$$

by conjugation. If we restrict this action to the subgroup $H \cap K$, the representation space (2.46) will split into a direct sum, which contains the slice representation of $H \cap K$ on $\mathfrak{h}^{\perp} \cap \mathfrak{k}^{\perp}$ as a summand. To identify $\mathfrak{h}^{\perp} \cap \mathfrak{k}^{\perp}$, we make the following remark. Consider the complex representation of $\operatorname{Sp}(1)$ which is the tensor product of the standard representation (highest weight (1)) by the adjoint representation (highest weight (2)). This representation has complex dimension $2 \times 3=6$. It contains as an irreducible summand the representation of highest weight (3) and complex dimension 4. From this, it follows that (2.46), if restricted to $H \cap K$, contains as an irreducible summand the tensor product of the 4-dimensional representation of $\mathrm{Sp}(1)$ by the standard representation of $\mathrm{Sp}(\mathrm{p}-1)$. This module has real dimension $8(p-1)$ and must therefore be equal to $\mathfrak{h}^{\perp} \cap \mathfrak{k}^{\perp}$ by a dimension count. By [DADOK], this is not a polar representation.

Case 3.2.2. Consider the case where $q \geq 2$.

$n=1$. We have the action of $H$ on the sphere $\mathrm{S}^{4 \mathrm{pq}-1} \subset \mathbb{R}^{4 \mathrm{pq}}$. Recall that this action is equivalent to the isotropy representation of $\operatorname{Sp}(p+q) / S p(p) \times S p(q)$. It can only be hyperpolar if it is of cohomogeneity one, which is the case iff $p=2$ or $q=2$.

$n=2$ or 3 . It can be deduced from (2.33) that $\mathfrak{h}^{\perp} \cap \mathfrak{k}^{\perp}$ contains as a direct summand the module

$$
\mathbb{R}^{2} \otimes \mathbb{H}^{(p-1) \times(q-1)} \text { or } \mathbb{R}^{3} \otimes \mathbb{H}^{(p-1) \times(q-1)}
$$

of the group $H \cap K$, which is locally isomorphic to $\mathrm{SO}(2)^{2} \times \mathrm{Sp}(\mathrm{p}-1) \times \mathrm{Sp}(\mathrm{q}-1)$, if $n=2$, or $\mathrm{SO}(3) \times \mathrm{Sp}(\mathrm{p}-1) \times \mathrm{Sp}(\mathrm{q}-1)$, if $n=3$, respectively. By [DADOK], these representations are not polar if $p \geq 3$ or $q \geq 3$. The case $p=2=q$ may be excluded by dimension conditions.

$n>4$. The slice representation is not polar by Lemma 2.9]

Result. The action of $\mathrm{Sp}(\mathrm{p}) \otimes \mathrm{Sp}(\mathrm{q})$ on the Grassmannian $\mathrm{SO}(4 \mathrm{pq}) / \mathrm{SO}(\mathrm{n}) \times$ $\mathrm{SO}(4 \mathrm{pq}-\mathrm{n})$ is hyperpolar iff

- $p q=2$ (Hermann action on $\mathrm{SO}(8))$,

- $\min (p, q)=2$ and $n=1$ (isotropy representation of a rank two symmetric space acting on a sphere), or

- $\min (p, q)=1, p q \neq 1$ and $n=2$. 
2.3.3. The Action of $\operatorname{Spin}(9)$ on $\mathrm{G}_{3}\left(\mathbb{R}^{16}\right)$. In the following we will show that the action of $H=\operatorname{Spin}(9)$ on the Grassmannian of 3-planes in $\mathbb{R}^{16}$, denoted by $\mathrm{G}_{3}\left(\mathbb{R}^{16}\right) \cong$ $\mathrm{SO}(16) / \mathrm{SO}(3) \times \mathrm{SO}(13)=\mathrm{G} / \mathrm{K}$, is not hyperpolar. This fact cannot be proved by computing or estimating the cohomogeneity of the action, because the cohomogeneity is $3=\operatorname{rk}\left(\mathrm{G}_{3}\left(\mathbb{R}^{16}\right)\right)$. Therefore, we need an explicit description of the spin representation of $\mathfrak{s o}(9)$ in order to determine a slice representation. We will prove that this slice representation is not polar, thus the action of $\operatorname{Spin}(9)$ on $G_{3}\left(\mathbb{R}^{16}\right)$ is not (hyper)polar.

For the construction of the spin representation see [LM], Chapter I. Using the formulae given there, one can explicitly write the natural embedding

$$
\mathbb{R}^{8} \hookrightarrow \mathrm{C} \ell_{8} \cong \mathbb{R}(16)
$$

for an orthonormal basis $e_{1}, \ldots, e_{8}$ of $\mathbb{R}^{8}$. By a suitable choice of basis elements, we may assume that the images of $e_{2}, \ldots, e_{8}$ are in $\left(\begin{array}{cc}\mathbb{R}(8) & 0 \\ 0 & \mathbb{R}(8)\end{array}\right)$, whereas the image of $e_{1}$ lies in $\left(\begin{array}{cc}0 & \mathbb{R}(8) \\ \mathbb{R}(8) & 0\end{array}\right)$.

The map $\mathfrak{s o}(9) \hookrightarrow \mathrm{C} \ell_{8}$ is given by

$$
e_{i} \wedge e_{j} \mapsto\left\{\begin{array}{cl}
\frac{1}{2} e_{i} \cdot e_{j}, & 1 \leq i<j \leq 8 ; \\
\frac{1}{2} e_{i}, & 1 \leq i \leq 8, j=9 .
\end{array}\right.
$$

In particular, for elements of the form $e_{1} \wedge v, v \in \mathbb{R}^{9}$ we have

$$
e_{1} \wedge\left(x_{2} e_{2}+\cdots+x_{9} e_{9}\right) \mapsto \frac{1}{2} e_{1} \cdot\left(x_{2} e_{2}+\cdots+x_{8} e_{8}+x_{9}\right) .
$$

A non-zero element $e_{1} \cdot\left(x_{2} e_{2}+\cdots+x_{8} e_{8}+x_{9}\right) \in \mathrm{C} \ell_{8}$ is invertible, its inverse being

$$
e_{1} \cdot\left(-x_{2} e_{2}-\cdots-x_{8} e_{8}-x_{9}\right) /\left(x_{2}^{2}+\cdots+x_{9}^{2}\right) \text {. }
$$

But these elements are mapped to $\left(\begin{array}{cc}0 & \mathbb{R}(8) \\ \mathbb{R}(8) & 0\end{array}\right)$, thus they are not contained in $\mathfrak{k}=$ $\mathfrak{s o}(3) \oplus \mathfrak{s o}(13)$, because otherwise the first three rows of the matrix representation of these elements were zero, which contradicts the fact that the matrices are invertible. From this, it follows that $\mathfrak{h} \cap \mathfrak{k}$ is contained in the subalgebra $\mathfrak{s p i n}(8) \subset \mathfrak{s p i n}(9)$, spanned by the elements $e_{i} \wedge e_{j}$, where $2 \leq i<j \leq 9$.

Furthermore, this subalgebra is the Lie algebra of a subgroup $\operatorname{Spin}(8) \subset \operatorname{Spin}(9)$, given by the direct sum of two 8-dimensional inequivalent representations of $\operatorname{Spin}(8)$, i.e. as a subgroup of $\mathrm{SO}(16), \operatorname{Spin}(8)$ is given by

$$
\operatorname{Spin}(8) \hookrightarrow \mathrm{SO}(16), \quad \mathrm{g} \mapsto\left(\begin{array}{cc}
\phi_{1}(g) & 0 \\
0 & \phi_{2}(g)
\end{array}\right),
$$

where $\phi_{1}$ and $\phi_{2}$ are two inequivalent, non-trivial 8-dimensional representations of $\operatorname{Spin}(8)$. From (2.51) it follows that $H \cap K=\phi_{1}^{-1}(\mathrm{~S}(\mathrm{O}(3) \times \mathrm{O}(5))) \subset \mathrm{Spin}(8)$. Since $\phi_{2}$ is inequivalent to $\phi_{1}$, it follows that $\phi_{2}(H \cap K)_{0}=\operatorname{Sp}(1) \otimes \operatorname{Sp}(2)$ or $\operatorname{Sp}(2) \otimes \operatorname{Sp}(1)$; see 3.1 .2 .

It remains to determine the representation module $\mathfrak{h}^{\perp} \cap \mathfrak{k}^{\perp}$ of $H \cap K$. Since $\operatorname{dim}(H \cap K)=13$, we already know that $\operatorname{dim}\left(\mathfrak{h}^{\perp} \cap \mathfrak{k}^{\perp}\right)=16$. Consider the space

$$
\mathfrak{k}^{\perp}=\left\{\left(\begin{array}{ccc}
0 & N & M \\
-N^{t} & 0 & 0 \\
-M^{t} & 0 & 0
\end{array}\right) \mid \begin{array}{c}
N \in \mathbb{R}^{3 \times 5} \\
M \in \mathbb{R}^{3 \times 8}
\end{array}\right\} .
$$


Since $\mathfrak{h}$ contains the subalgebra $\mathfrak{s p i n}(8)$ as described above, it is clear that $N=0$ in (2.52) for elements of $\mathfrak{h}^{\perp} \cap \mathfrak{k}^{\perp}$. Thus, $\mathfrak{h}^{\perp} \cap \mathfrak{k}^{\perp}$ is a subspace of

$$
\left\{\left(\begin{array}{cc}
0 & M \\
-M^{t} & 0
\end{array}\right) \mid M \in \mathbb{R}^{3 \times 8}\right\}
$$

and $(H \cap K)_{0}$ is a subgroup of

$$
\left\{\left(\begin{array}{ll}
A & \\
& B
\end{array}\right) \mid \begin{array}{l}
A \in \mathrm{SO}(3) \times \mathrm{SO}(5) \\
B \in \mathrm{Sp}(1) \otimes \mathrm{Sp}(2) \subset \mathrm{SO}(8)
\end{array}\right\}
$$

which acts on (2.53) by conjugation. A dimension count shows that the slice representation is equivalent to the tensor product of the 4-dimensional irreducible representation of $\operatorname{Sp}(1)$ and the standard representation of $\operatorname{Sp}(2)$. This representation is not polar by [DADOK].

2.4. The Classification. We start with the classification of the hyperpolar actions on the classical Lie groups. Let $G$ be a classical Lie group $G=\mathrm{SO}(\mathrm{n}), \mathrm{SU}(\mathrm{n})$ or $\mathrm{Sp}(\mathrm{n})$. By Lemma 2.8 it is sufficient to consider such pairs $(H, K)$ of subgroups of $G$, where $K \subset G$ is a symmetric subgroup (with one exception). The corresponding symmetric spaces $G / K$ are the real, complex and quaternionic Grassmannians and the spaces

$$
\mathrm{SO}(2 \mathrm{~m}) / \mathrm{U}(\mathrm{m}), \quad \mathrm{SU}(\mathrm{n}) / \mathrm{SO}(\mathrm{n}), \quad \mathrm{SU}(2 \mathrm{~m}) / \mathrm{Sp}(\mathrm{m}), \quad \mathrm{Sp}(\mathrm{n}) / \mathrm{U}(\mathrm{n}) .
$$

By Theorem 2.2, the maximal subgroups of the classical groups are either symmetric subgroups, certain tensor products or simple irreducible subgroups. The symmetric subgroups lead to Hermann actions and the tensor product actions on Grassmannians were already discussed. By Lemma 2.8 the tensor product actions on the spaces (2.54) are not hyperpolar.

Thus it remains to consider the simple irreducible subgroups $H$ of $\mathrm{SO}(\mathrm{n}), \mathrm{SU}(\mathrm{n})$ and $\mathrm{Sp}(\mathrm{n})$, which are given by irreducible representations of the real, complex or quaternionic type, respectively. Fortunately, we do not have to consider the actions of $H \subset G$ on all quotients $G / K$ by symmetric subgroups $K \subset G$, because of the following remark: In the case where $M=G / K$ is a symmetric space, (2.3) is a necessary condition for hyperpolar actions. But for the Grassmannians we have the following inequality, where we define $\nu(M) \equiv \operatorname{dim}(M)-\operatorname{rk}(\mathrm{M})$ :

$$
\nu\left(\mathrm{G}_{\mathrm{k}}\left(\mathbb{K}^{\mathrm{n}}\right)\right) \leq \nu\left(\mathrm{G}_{\ell}\left(\mathbb{K}^{\mathrm{n}}\right)\right) \quad \text { if } \quad 1 \leq \mathrm{k}<\ell \leq \frac{\mathrm{n}}{2},
$$

where $\mathbb{K}=\mathbb{R}, \mathbb{C}$ or $\mathbb{H}$. In most cases, condition (2.3) is violated already for $G / K=$ $\mathrm{G}_{1}\left(\mathbb{K}^{\mathrm{n}}\right)$ or $\mathrm{G}_{2}\left(\mathbb{K}^{\mathrm{n}}\right)$, and hence, by inequality (2.55), also for the Grassmannians of higher rank. Further, in many cases it follows from Lemma 2.8 that $H$ does not act hyperpolarly on the spaces (2.54).

In some cases, $H \times K$ acts transitively on $G$. Then we have to consider the actions of maximal connected subgroups of $H \times K$ on $G$. This will be done in 2.4.5

2.4.1. Hyperpolar Actions on $\mathrm{SO}(n)(n \geq 7)$. We will now consider the $H \times K$ actions on $\mathrm{SO}(\mathrm{n})$, where $H$ and $K$ are maximal connected subgroups of $\mathrm{SO}(\mathrm{n})$. By Lemma 2.8 we may assume that $K$ is a symmetric subgroup or $K=\mathrm{G}_{2} \subset \mathrm{SO}(7)$. We take the maximal connected subgroups $H \subset \mathrm{SO}(\mathrm{n})$ from Theorem [2.2. In all five cases, we will decide which of the corresponding subgroups act hyperpolarly on $\mathrm{SO}(\mathrm{n}) / \mathrm{SO}(\mathrm{m}) \times \mathrm{SO}(\mathrm{n}-\mathrm{m})$ or $\mathrm{SO}(\mathrm{n}) / \mathrm{U}\left(\frac{\mathrm{n}}{2}\right)$. Actions on $\mathrm{SO}(7) / \mathrm{G}_{2}$ will be considered at the end of 2.4 .1 
We start with the subgroups of type (i) and (iii). They are symmetric and one obtains Hermann actions in these cases.

The actions of the subgroups (ii) and (iv) on the real Grassmannians were already investigated in 2.3.1 and 2.3.2 Lemma[2.8] shows that they do not act hyperpolarly on $\mathrm{SO}(\mathrm{n}) / \mathrm{U}\left(\frac{\mathrm{n}}{2}\right)$.

It remains the case of irreducible simple subgroups, i.e. the subgroups of type (v). The ones that are not a priori excluded because their dimensions are too low are given in Lemma 2.6.

From Lemma 2.8 it follows that we only have to study the actions of these groups on the Grassmannians $\mathrm{SO}(\mathrm{n}) / \mathrm{SO}(\mathrm{k}) \times \mathrm{SO}(\mathrm{n}-\mathrm{k})$. We will now consider case-by-case each of these simple irreducible subgroups; they are given by the table in part i) of Lemma 2.8 With each of these subgroups $H \subset \mathrm{SO}(\mathrm{n})$ we proceed as follows.

We start by considering the action of $H$ on $\mathrm{G}_{1}\left(\mathbb{R}^{\mathrm{n}}\right)=\mathrm{SO}(\mathrm{n}) / \mathrm{SO}(\mathrm{n}-1)$, i.e. on the sphere. Since the spheres are rank one symmetric spaces, these actions are hyperpolar iff $H$ acts with cohomogeneity one. It is well known that in this case $H$ is given by the isotropy representation of a rank-two symmetric space; see [DADOK].

Then we move on to the Grassmannians of two-planes $\mathrm{G}_{2}\left(\mathbb{R}^{\mathrm{n}}\right)=\mathrm{SO}(\mathrm{n}) / \mathrm{SO}(\mathrm{n}-$ 2) $\times \mathrm{SO}(2)$. In all but two cases it follows already from the dimension condition (2.3) that $H$ does not act hyperpolarly on $\mathrm{G}_{2}\left(\mathbb{R}^{\mathrm{n}}\right)$, and hence, by (2.55)), also not on the other Grassmannians $\mathrm{G}_{3}\left(\mathbb{R}^{\mathrm{n}}\right), \ldots, \mathrm{G}_{\left\lfloor\frac{\mathrm{n}}{2}\right\rfloor}\left(\mathbb{R}^{\mathrm{n}}\right)$. The two remaining cases are

- $\mathrm{F}_{4}$ on $\mathrm{G}_{2}\left(\mathbb{R}^{26}\right)$,

- $\operatorname{Spin}(9)$ on $\mathrm{G}_{2}\left(\mathbb{R}^{16}\right)$.

The second action has cohomogeneity one. This may be seen as follows: It is well-known that $\operatorname{Spin}(9)$ acts transitively on the unit sphere in $\mathbb{R}^{16}$, where the isotropy group is $\operatorname{Spin}(7) \subset \operatorname{Spin}(8) \subset \operatorname{Spin}(9)$; see e.g. HsHS. The subgroup $\operatorname{Spin}(8) \subset \mathrm{SO}(16)$ is given by the sum of the two half-spin representations. Thus we may assume that $\operatorname{Spin}(7)$, as the isotropy group of the first canonical basis vector $e_{1} \in \mathbb{R}^{16}$ is given by the representation

$$
\mathrm{N} \oplus \varrho_{1} \oplus \varrho_{3},
$$

where $\mathrm{N}$ denotes a one-dimensional trivial representation, $\varrho_{1}$ is the 7 -dimensional standard representation, and $\varrho_{3}$ is the 8-dimensional spin representation of $\operatorname{Spin}(7)$. This group acts with cohomogeneity one on the unit sphere in $\left\{e_{1}\right\}^{\perp}$, its principal orbits being cartesian products of 6 -spheres by 7 -spheres. This shows that $\operatorname{Spin}(9)$ acts with cohomogeneity one on the space of pairs of orthogonal unit vectors in $\mathbb{R}^{16}$, i.e. on the Stiefel manifold $V_{2}\left(\mathbb{R}^{16}\right)$. Since the action of $\operatorname{Spin}(9)$ on $G_{2}\left(\mathbb{R}^{16}\right)$ is not transitive (see ON1]) it must be of cohomogeneity one.

Now consider the action of $\mathrm{F}_{4}$ on $\mathrm{G}_{2}\left(\mathbb{R}^{26}\right)$. We will show that this action is not hyperpolar because its cohomogeneity is greater than or equal to 3 . Consider the subgroup $\operatorname{Spin}(8) \subset \operatorname{Spin}(9) \subset \mathrm{F}_{4}$. If the action of $\mathrm{F}_{4}$ on $\mathbb{R}^{26}$ is restricted to Spin(8), a two-dimensional subspace $V \subset \mathbb{R}^{26}$ is left invariant, because the only irreducible representations of $\operatorname{Spin}(8)$ whose degree is less than or equal to 26 , are the 8-dimensional representations. Let us determine the connected component of the isotropy group $\left(H_{V}\right)_{0}$ of $V \in \mathrm{G}_{2}\left(\mathbb{R}^{26}\right)$ in $H=\mathrm{F}_{4}$. Since $H_{V}$ contains $\operatorname{Spin}(8)$, its rank is 4 . Thus it is itself contained in one of the maximal connected subgroups of $\mathrm{F}_{4}$ of maximal rank. These are given, e.g. in [ON2], Chapter 1, $\S 3$, Theorem 16, pp. $63-64$ :

$$
\mathrm{Sp}(1) \cdot \mathrm{Sp}(3), \quad \mathrm{SU}(3) \cdot \mathrm{SU}(3), \quad \mathrm{Spin}(9) .
$$


The only possibility is $\left(H_{V}\right)_{0} \subseteq \operatorname{Spin}(9)$, since $\left(H_{V}\right)_{0}$ contains the 28-dimensional subgroup $\operatorname{Spin}(8)$. Now we have $\operatorname{Spin}(8) \subseteq\left(\mathrm{H}_{\mathrm{V}}\right)_{0} \subseteq \operatorname{Spin}(9)$. But $\operatorname{Spin}(8) \subset \operatorname{Spin}(9)$ is a maximal connected subgroup, thus either $\left(H_{V}\right)_{0}=\operatorname{Spin}(8)$ or $\left(H_{V}\right)_{0}=\operatorname{Spin}(9)$. But from Table 25, p. 199, in DYN1] one can read off that $\mathrm{F}_{4} \subset \mathrm{SO}(26)$, restricted to $\operatorname{Spin}(9)$ acts on $\mathbb{R}^{26}$ by the representation

$$
\varrho_{1} \oplus \varrho_{4} \oplus \mathrm{N}
$$

where $\varrho_{1}$ is the 9 -dimensional standard representation, $\varrho_{4}$ is the 16 -dimensional spin representation of $\operatorname{Spin}(9)$ and $\mathrm{N}$ denotes a one-dimensional trivial representation. Hence $\operatorname{Spin}(9)$ does not leave invariant the two-dimensional subspace $V$. Thus $\left(H_{V}\right)_{0}=\operatorname{Spin}(8)$ and the normal space to the orbit of the $\mathrm{F}_{4}$-action on $\mathrm{G}_{2}\left(\mathbb{R}^{26}\right)$ in $V$ is 24-dimensional. From the low-dimensional representations of $\operatorname{Spin}(8)$ it is clear that $\operatorname{Spin}(8)$ acts at least with cohomogeneity 3 on the normal space.

Now we move on to actions of the two groups given above on Grassmannians of three-planes. It is clear from (2.3) that $\mathrm{F}_{4}$ cannot act hyperpolarly on $\mathrm{G}_{3}\left(\mathbb{R}^{26}\right)$. The action of $\operatorname{Spin}(9)$ on $\mathrm{G}_{3}\left(\mathbb{R}^{16}\right)$ was shown not to be hyperpolar in 2.3.3. Now it follows again from (2.3) and (2.55) that there can be no hyperpolar action of these groups on the Grassmannians of higher rank.

Actions on $\mathrm{SO}(7)$. We also have to check if actions of maximal connected subgroups of $\mathrm{SO}(7)$ act hyperpolarly on $\mathrm{SO}(7) / \mathrm{G}_{2}$; see Lemma 2.8. The maximal connected subgroups of $\mathrm{SO}(7)$ not excluded by (2.3) are, by Theorem 2.2 and the Appendix:

$$
\mathrm{SO}(6), \quad \mathrm{SO}(5) \times \mathrm{SO}(2), \quad \mathrm{SO}(4) \times \mathrm{SO}(3), \quad \mathrm{G}_{2} .
$$

The first two groups act transitively on $\mathrm{SO}(7) / \mathrm{G}_{2}$.

The group $\mathrm{SO}(4) \times \mathrm{SO}(3)$ acts with cohomogeneity one on $\mathrm{SO}(7) / \mathrm{G}_{2}$. This can be seen as follows: Consider the action of $G_{2}$ on the Stiefel manifold $V_{3}\left(\mathbb{R}^{7}\right)$. It is well-known that $\mathrm{G}_{2}$ acts transitively on the Stiefel manifold $\mathrm{V}_{2}\left(\mathbb{R}^{7}\right)$ (see [ON1]); the isotropy group of $\left(e_{1}, e_{2}\right) \in \mathrm{V}_{2}\left(\mathbb{R}^{7}\right)$ under this action is $\mathrm{SU}(2) \subset \mathrm{SU}(3) \subset \mathrm{G}_{2}$. The group $\mathrm{SU}(2)$ acts on the unit sphere in $\left\{e_{1}, e_{2}\right\}^{\perp} \cong \mathbb{R}^{5}$ with cohomogeneity one. From this, it follows that $\mathrm{G}_{2}$ acts on $V_{3}\left(\mathbb{R}^{7}\right)$ with cohomogeneity one, and since $\mathrm{G}_{2}$ does not act transitively on $\mathrm{G}_{3}\left(\mathbb{R}^{7}\right)$ (see ON1]) it acts with cohomogeneity one.

Finally, the action of $\mathrm{G}_{2}$ on $\mathrm{S}^{7}=\mathrm{SO}(7) / \mathrm{G}_{2}$ is of cohomogeneity one; see HPTT2.

2.4.2. Hyperpolar Actions on $\mathrm{SU}(n)(n \geq 2)$. We proceed in the same way as in 2.4.1 By Lemma 2.8, we only have to consider the actions of maximal connected subgroups $H$ on the spaces $\mathrm{SU}(\mathrm{n}) / \mathrm{K}$, where $K \subset \mathrm{SU}(\mathrm{n})$ is a symmetric subgroup, i.e. on the complex Grassmannians and on the spaces $\mathrm{SU}(\mathrm{n}) / \mathrm{SO}(\mathrm{n})$ and $\mathrm{SU}(\mathrm{n}) / \mathrm{Sp}\left(\frac{\mathrm{n}}{2}\right)$. The maximal connected subgroups $H \subset \mathrm{SU}(\mathrm{n})$ are given in Theorem 2.2 .

The subgroups (i), (ii) and (iii) correspond to the various Hermann actions on $\mathrm{SU}(\mathrm{n})$.

Case (iv) is treated in 2.3.1.

It remains to study the actions of certain simple irreducible subgroups. The actions given by Lemma [2.6, part iv), can be shown not to act hyperpolarly by condition (2.3).

The remaining subgroups are described in Lemma 2.6, case ii), i.e. up to a U(1)factor, they are isotropy actions of Hermitian symmetric spaces. By (2.3) and (2.55), we only have to consider the actions of these groups on complex projective 
space $G_{1}\left(\mathbb{C}^{n}\right)$. It is clear that the cohomogeneity of these actions is the rank of the corresponding symmetric space minus one. Since $\operatorname{rk}\left(\mathrm{G}_{1}\left(\mathbb{C}^{\mathrm{n}}\right)\right)=1$, these actions are only hyperpolar if they are of cohomogeneity one, i.e. if they come from isotropy actions of rank two symmetric spaces; cf. [TAKAGI.

2.4.3. Hyperpolar Actions on $\operatorname{Sp}(n)(n \geq 2)$. Consider the maximal connected subgroups $H \subset \mathrm{Sp}(\mathrm{n})$ given in Theorem 2.2 We will decide if they act hyperpolarly on the spaces $\operatorname{Sp}(\mathrm{n}) / \mathrm{K}$, where $K$ is a symmetric subgroup, i.e. on the quaternionic Grassmannians and on the spaces $\operatorname{Sp}(\mathrm{n}) / \mathrm{U}(\mathrm{n})$.

Subgroups i) and ii) correspond to Hermann actions; the subgroups of type iii) were studied in 2.3 .1

It remains the case of simple subgroups given by irreducible representations of quaternionic type; see Lemma 2.6 With the exception of the spin representation of $\mathrm{B}_{5}$, which can be excluded by condition (2.3), these representations are as described in Lemma 2.6] case iii), i.e. up to a $\mathrm{Sp}(1)$-factor, they are isotropy representations of Quaternion-Kähler symmetric spaces. These isotropy representations may be thought of as quaternionic matrices acting on the space of column vectors $\mathbb{H}^{n}$ by left multiplication, whereas the group $\operatorname{Sp}(1)$ acts by right multiplication. Quaternionic projective space $\mathbb{H} \mathrm{P}^{n-1}$ is defined as the quotient of the sphere $\mathrm{S}^{4 \mathrm{n}-1} \subset \mathbb{H}^{\mathrm{n}}$ by the equivalence relation

$$
v \sim w \text { iff there exists } q \in \operatorname{Sp}(1) \text {, such that } v=w q .
$$

From this, it follows that the cohomogeneity of the $H$-action on $\mathbb{H} \mathrm{P}^{n-1}$ is equal to the rank of the corresponding symmetric space minus one. Thus we have a hyperpolar action if $H$ is given by the isotropy representation of a rank two QuaternionKähler symmetric space which (for simple $H$ ) is the case only for the isotropy representation of $\mathrm{G}_{2} / \mathrm{SO}(4)$; cf. [D'ATRI. By (2.3) and (2.55), the actions of the simple irreducible subgroups on the other Grassmannians are not hyperpolar.

2.4.4. Hyperpolar Actions on the Exceptional Lie Groups. To find all the hyperpolar actions on the exceptional Lie groups, we follow the same steps as in the case of the classical groups. Let $G$ be an exceptional Lie group, i.e. $G=\mathrm{E}_{6}, \mathrm{E}_{7}, \mathrm{E}_{8}, \mathrm{~F}_{4}, \mathrm{G}_{2}$. From Table 3 we take the maximal dimension of a proper closed subgroup of $G$. From (2.5) we obtain a lower bound on the dimensions of subgroups $H$ and $K$ : We only have to consider maximal subgroups of $\mathrm{E}_{6}, \mathrm{E}_{7}, \mathrm{E}_{8}, \mathrm{~F}_{4}$, and $\mathrm{G}_{2}$ whose dimensions are greater than or equal to $20,47,104,12$ or 4 , respectively. These subgroups may be taken from [DYN1], Table 12, p. 150, Table 12a, p. 151 and ON2], §3, Thm. 16. They are

\begin{tabular}{|l|l|}
\hline$G$ & Subgroups \\
\hline $\mathrm{E}_{6}$ & $\mathrm{SU}(6) \cdot \mathrm{SU}(2), \mathrm{Spin}(10) \cdot \mathrm{SO}(2), \mathrm{C}_{4}^{1}, \mathrm{~F}_{4}^{1}, \mathrm{SU}(3) \cdot \mathrm{SU}(3) \cdot \mathrm{SU}(3), \mathrm{G}_{2}^{1} \cdot \mathrm{A}_{2}^{2^{\prime \prime}}$ \\
\hline $\mathrm{E}_{7}$ & $\mathrm{SO}^{\prime}(12) \cdot \mathrm{SU}(2), \mathrm{SU}(8) /\{ \pm 1\}, \mathrm{E}_{6} \cdot \mathrm{U}(1), \mathrm{F}_{4}^{1} \cdot \mathrm{A}_{1}^{3^{\prime \prime}}$ \\
\hline $\mathrm{E}_{8}$ & $\mathrm{SO}^{\prime}(16), \mathrm{E}_{7} \cdot \mathrm{SU}(2)$ \\
\hline $\mathrm{F}_{4}$ & $\mathrm{Sp}(3) \cdot \mathrm{Sp}(1), \mathrm{SU}(3) \cdot \mathrm{SU}(3), \mathrm{Spin}(9), \mathrm{G}_{2}^{1} \cdot \mathrm{A}_{1}^{8}$ \\
\hline $\mathrm{G}_{2}$ & $\mathrm{SU}(3), \mathrm{SO}(4)$ \\
\hline
\end{tabular}


Actions on $\mathrm{E}_{6}$. The only action that is neither a Hermann action nor excluded by dimension conditions is the action of $H=\mathrm{SU}(3) \cdot \mathrm{SU}(3) \cdot \mathrm{SU}(3)$ on $G / K=\mathrm{E}_{6} / \mathrm{F}_{4}$. If this action is hyperpolar, it is of cohomogeneity two because $\operatorname{dim}(\mathrm{SU}(3) \cdot \mathrm{SU}(3)$. $\mathrm{SU}(3))=24, \operatorname{dim}\left(\mathrm{E}_{6} / \mathrm{F}_{4}\right)=26$ and $\operatorname{rk}\left(\mathrm{E}_{6} / \mathrm{F}_{4}\right)=2$. By conjugation, we may assume that $\operatorname{rk}(H \cap K)=4$. Since the Lie algebra $\mathfrak{h} \cap \mathfrak{k}$ is a subalgebra of $\mathfrak{h}=3 \mathfrak{a}_{2}$, it is isomorphic to

$$
k \mathbb{R} \oplus \ell \mathfrak{a}_{1} \oplus m \mathfrak{a}_{2}, \text { where } k+\ell+2 m=4 .
$$

From this it follows that the dimension of the slice representation of $\mathfrak{h} \cap \mathfrak{k}$ is $k+$ $3 \ell+8 m+2$. It may be easily verified that there is no such representation of cohomogeneity two.

Actions on $\mathrm{E}_{7}$. In the case $G=\mathrm{E}_{7}$, the only non-symmetric subgroup in (2.57) is $H=\mathrm{F}_{4}^{1} \cdot \mathrm{A}_{1}^{3 \prime \prime}$. By the dimension condition (2.3), all actions of this group on quotients of $\mathrm{E}_{7}$ are excluded, except the action on $G / K=\mathrm{E}_{7} / \mathrm{E}_{6} \cdot \mathrm{U}(1)$. We will show that this action is not hyperpolar by considering a slice representation. First we determine the isotropy group of the identity element, $H \cap K$. From Table 25 in DYN1], p. 204, one sees that, after conjugation, the group $\mathrm{F}_{4}^{1}$ is contained in the subgroup $\mathrm{E}_{6}$. Thus we may assume $\mathrm{F}_{4}^{1} \subseteq \mathrm{H} \cap \mathrm{K}$. On the other hand, $H \cap K \neq \mathrm{F}_{4}^{1} \cdot \mathrm{A}_{1}^{3 \prime \prime}$, because $K \subset G$ is maximal connected. Hence $(H \cap K)_{0}=\mathrm{F}_{4}^{1}$ or $\mathrm{F}_{4}^{1} \cdot \mathrm{U}(1)$ and the normal space at the identity element is 51- or 52-dimensional, respectively. But by [DADOK], the only suitable polar representation of $H \cap K$ is the adjoint representation of $\mathrm{F}_{4}$, which is of cohomogeneity $4>\operatorname{rk}\left(\mathrm{E}_{7} / \mathrm{E}_{6} \cdot \mathrm{U}(1)\right)=3$. Thus the action is not hyperpolar.

Actions on $\mathrm{E}_{8}$. Since the subgroups of $\mathrm{E}_{8}$ listed in (2.57) are both symmetric, we conclude that all hyperpolar actions on $\mathrm{E}_{8}$ are Hermann actions or $\sigma$-actions.

Actions on $\mathrm{F}_{4}$. We only have to check if the actions of $H=\mathrm{G}_{2}^{1} \cdot \mathrm{A}_{1}^{8}$ or $\mathrm{SU}(3) \cdot \mathrm{SU}(3)$ on $G / K=\mathrm{F}_{4} / \operatorname{Spin}(9)$ are hyperpolar, because the other combinations are either Hermann actions or can be excluded by the dimension condition (2.4).

First let $H=\mathrm{G}_{2}^{1} \cdot \mathrm{A}_{1}^{8}$. By an analogous argument as in the preceding paragraph, one may assume that $H \cap K=\mathrm{G}_{2}^{1}$ or $\mathrm{G}_{2}^{1} \cdot \mathrm{U}(1)$; see Table 25 in [DYN1], p. 199. Thus the normal space is 13 - or 14-dimensional, respectively. But there is no suitable representation of $H \cap K$ of cohomogeneity one. Thus the cohomogeneity of the $H$-action on $G / K$ is at least two and hence the $H$-action is not hyperpolar.

Now let $H=\mathrm{SU}(3) \cdot \mathrm{SU}(3), K=\operatorname{Spin}(9), G=\mathrm{F}_{4}$. Consider the action of $H \times K$ on $G$. If this action is hyperpolar, it is of cohomogeneity one because $\operatorname{rk}(\mathrm{G} / \mathrm{K})=1$. Both subgroups $H$ and $K$ are subgroups of maximal rank in $G$, thus one may assume that they have a common maximal torus. From this it follows that the root systems of $H$ and $K$ are subsets of the root system of $G$; see ON2, $\S 3$, Theorem 16. In this case it is easy to determine the Lie algebra $\mathfrak{h} \cap \mathfrak{k}$ of the stabilizer of $e \in G$ : Its complexification $(\mathfrak{h} \cap \mathfrak{k})_{\mathbb{C}} \subset \mathfrak{g}_{\mathbb{C}}$ is spanned by the complexification of the Cartan subalgebra and those root spaces which correspond to the roots that are in the intersection of the root systems of $\mathfrak{h}$ and $\mathfrak{k}$. We may take the positive roots of $\mathrm{F}_{4}$ from [TITS] and the root subsystems of $\mathfrak{h}$ and $\mathfrak{k}$ from [ON2], §3, Theorem 16. We obtain $\operatorname{dim}(\mathfrak{h} \cap \mathfrak{k})$ and from this $\operatorname{dim}\left(\mathfrak{h}^{\perp} \cap \mathfrak{k}^{\perp}\right)$. It follows from the classification of the groups transitive on spheres that $H \cap K$ does not act with cohomogeneity one on $\mathfrak{h}^{\perp} \cap \mathfrak{k}^{\perp}$. Hence the $H \times K$-action on $G$ is not hyperpolar. 
Actions on $\mathrm{G}_{2}$. It is well-known that the action of $\mathrm{SU}(3)$ on $\mathrm{G}_{2} / \mathrm{SU}(3)$ is of cohomogeneity one; see e.g. [HPTT2.

Finally, it is easy to see that the action of $H=\mathrm{SO}(4)$ on $G / K=\mathrm{G}_{2} / \mathrm{SU}(3)=\mathrm{S}^{6}$ is of cohomogeneity one.

2.4.5. Transitive Subgroups. We will now consider maximal subgroups of transitive subgroups. In [ON1], all pairs $(H, K)$ of proper connected subgroups of the connected simple Lie groups $G$ such that $G=H \cdot K$ were classified. The result may be summarized by Table 4 .

\begin{tabular}{|c|c|c|c|}
\hline No. & $H$ & $G$ & $K$ \\
\hline \hline 1. & $\mathrm{Sp}(\mathrm{n})$ & $\mathrm{SU}(2 \mathrm{n})$ & $\mathrm{S}_{\left(\mathrm{U}_{2 n-1} \times \mathrm{U}_{1}\right)}$ \\
\hline 2. & $\mathrm{SO}(2 \mathrm{n}-1)$ & $\mathrm{SO}(2 \mathrm{n})$ & $\mathrm{U}(\mathrm{n})$ \\
\hline 3. & $\mathrm{SO}(4 \mathrm{n}-1)$ & $\mathrm{SO}(4 \mathrm{n})$ & $\mathrm{Sp}(\mathrm{n}) \cdot \mathrm{Sp}(1)$ \\
\hline 4. & $\mathrm{G}_{2}$ & $\mathrm{SO}(7)$ & $\mathrm{SO}(6)$ \\
\hline 5. & $\mathrm{G}_{2}$ & $\mathrm{SO}(7)$ & $\mathrm{SO}(5) \times \mathrm{SO}(2)$ \\
\hline 6. & $\mathrm{Spin}(7)$ & $\mathrm{SO}(8)$ & $\mathrm{SO}(7)$ \\
\hline 7. & $\mathrm{Spin}(9)$ & $\mathrm{SO}(16)$ & $\mathrm{SO}(15)$ \\
\hline
\end{tabular}

TABLE 4. Transitive actions

Remarks. In the table, only the maximal connected subgroups $H, K$ having the property $G=H \cdot K$ are given. The complete list may be obtained as follows: If the group $K$ is a product of a group $K^{\prime}$ by a $\mathrm{U}(1)$ - or $\mathrm{Sp}(1)$-factor, then also $G=H \cdot K^{\prime}$; cf. Theorem 4.1 in ON1. The transitive actions are given only up to local conjugacy. Note that since the condition (2.4) is obviously satisfied for transitive actions, all transitive subgroups are found by our approach, i.e. we have actually reproved the result of [ON1] by our method.

We proceed as follows: For each transitive action of $H \times K$ on $G$ in Table 4 , we determine the maximal connected subgroups of $H \times K$ by Theorem 2.1. If $\mathfrak{h} \neq \mathfrak{k}$, Theorem 2.1 shows that the maximal connected subgroups of $H \times K$ are of the form $\hat{H} \times K$ or $H \times \hat{K}$, where $\hat{H}$ and $\hat{K}$ are maximal connected subgroups of $H$ and $K$, respectively. If $\mathfrak{h} \cong \mathfrak{k}$, there are also "diagonal" subgroups.

In many cases, a maximal connected subgroup $U \subset H \times K$ is contained in a nontransitive subgroup $U^{\prime} \subset G \times G$ and thus is not a maximal connected non-transitive subgroup of $G \times G$. We will treat the rows of Table 4 case-by-case.

1.a) Let $H=\mathrm{Sp}(\mathrm{n}), G=\mathrm{SU}(2 \mathrm{n}), K=\mathrm{S}\left(\mathrm{U}_{2 n-1} \times \mathrm{U}_{1}\right)$. The group $H$ acts transitively on the complex projective space $G / K$ with isotropy group $\operatorname{Sp}(\mathrm{n}-1)$. $\mathrm{U}(1)$; see $\mathrm{ON} 1]$. Thus $G / K \cong \mathrm{Sp}(\mathrm{n}) / \mathrm{Sp}(\mathrm{n}-1) \cdot \mathrm{U}(1)$. Assume $\hat{H}$ is a maximal connected subgroup of $H$, which acts hyperpolarly on $G / K$, i.e. with cohomogeneity one. Since $\operatorname{Sp}(\mathrm{n}-1) \cdot \mathrm{U}(1) \subset \mathrm{Sp}(\mathrm{n}-1) \times \mathrm{Sp}(1)$, it follows that $\hat{H}$ also acts on $\mathbb{H} \mathrm{P}^{\mathrm{n}-1}$ with cohomogeneity one or transitively. But, by Table 4 , there are no transitive actions on $\mathbb{H} \mathrm{P}^{\mathrm{n}-1}$ besides the standard action of $\mathrm{Sp}(\mathrm{n})$ and thus we have found all cohomogeneity one actions on $\mathbb{H} \mathrm{P}^{\mathrm{n}-1}=\mathrm{Sp}(\mathrm{n}) / \mathrm{Sp}(1) \times \mathrm{Sp}(\mathrm{n}-1)$ already in 2.4.3. they are: the Hermann actions of $\operatorname{Sp}(\mathrm{k}) \times \mathrm{Sp}(\mathrm{n}-\mathrm{k})$ and $\mathrm{U}(\mathrm{n})$, and an action given by the 4-dimensional irreducible representation of $\mathrm{A}_{1}$. The first two groups are contained in reducible symmetric subgroups of $G$, which do not act transitively on $G / K$. The third group may be excluded because its dimension is too low. 
1.b) Now consider the connected subgroups of $K$ that act non-transitively on $G / H$ and are maximal with respect to this property. They are of the form $K^{\prime} \cdot \mathrm{U}(1)$, where $K^{\prime} \subset \mathrm{SU}(2 \mathrm{n}-1)$ is a maximal connected subgroup. It is easy to verify that if inequality (2.13) holds for $K^{\prime}$, i.e. if $\operatorname{dim} K^{\prime}<\frac{1}{2}(2 n-1)^{2}-(2 n-1)$, then $K^{\prime} \cdot \mathrm{U}(1)$ cannot act hyperpolarly on $G / H$. Hence, by Lemma [2.8, part iv), we only have to consider symmetric subgroups $K^{\prime} \subset \mathrm{SU}(2 \mathrm{n}-1)$, i.e. $\mathrm{S}\left(\mathrm{U}_{k} \times \mathrm{U}_{2 n-1-k}\right)$, $1 \leq k<n$ and $\mathrm{SO}(2 \mathrm{n}-1)$. But these groups are contained in the symmetric subgroups $\mathrm{S}\left(\mathrm{U}_{k+1} \times \mathrm{U}_{2 n-1-k}\right)$ or $\mathrm{SO}(2 \mathrm{n})$, respectively, of $G=\mathrm{SU}(2 \mathrm{n})$.

2.a) Let $K=\mathrm{U}(\mathrm{n}), G / H=\mathrm{SO}(2 \mathrm{n}) / \mathrm{SO}(2 \mathrm{n}-1)$. We have to find those subgroups $K^{\prime}$ of $K$ which act with cohomogeneity one on the sphere. It is well-known that such actions are given by isotropy representations of rank-two symmetric spaces, if $K^{\prime}$ is not contained in another subgroup of $K$ which has the same orbits.

2.b) Now consider $G / K=\mathrm{SO}(2 \mathrm{n}) / \mathrm{U}(\mathrm{n})$. We have to determine the maximal connected subgroups of $H=\mathrm{SO}(2 \mathrm{n}-1)$ that act hyperpolarly on $G / K$. By an analogous argument as in 1.b), we only have to consider the subgroups of $\mathrm{SO}(2 \mathrm{n}-1)$ given by Lemma 2.8, part iv): $\mathrm{SO}(\mathrm{k}) \times \mathrm{SO}(2 \mathrm{n}-1-\mathrm{k}), 1 \leq k<n$ and $\mathrm{G}_{2} \subset$ $\mathrm{SO}(7)$. But these groups are contained in the symmetric subgroups $\mathrm{SO}(\mathrm{k}+1) \times$ $\mathrm{SO}(2 \mathrm{n}-\mathrm{k}-1) \subset \mathrm{SO}(2 \mathrm{n})$ and $\mathrm{Spin}(7) \subset \mathrm{SO}(8)$, respectively.

3.a) The subgroups of $K=\mathrm{Sp}(\mathrm{n}) \otimes \mathrm{Sp}(1)$ which act with cohomogeneity one on $G / H=\mathrm{SO}(4 \mathrm{n}) / \mathrm{SO}(4 \mathrm{n}-1)=\mathrm{S}^{4 \mathrm{n}-1}$ and are maximal with respect to this property are given by isotropy representations of certain rank-two symmetric spaces.

3.b) By the same argument as in 1.b) and 2.b), one can show that for subgroups $H^{\prime} \subset H$ either $H^{\prime} \times K$ does not act hyperpolarly on $G$ or $H^{\prime} \times K$ is not a maximal connected non-transitive subgroup of $G$.

4.a) The maximal connected subgroups of $\mathrm{G}_{2}$ are $\mathrm{SO}(4), \mathrm{SU}(3)$ and a group of local type $\mathrm{A}_{1}$; see DYN1. As subgroups of $\mathrm{SO}(7)$, the first two are reducible. The dimension of the third group is too low for a hyperpolar action.

4.b) Now consider the maximal connected subgroups of $\mathrm{SO}(6)$. The subgroup $\mathrm{SO}(5)$ will be considered in 5.b). The subgroups $\mathrm{SO}(2) \times \mathrm{SO}(4)$ and $\mathrm{SO}(3) \times \mathrm{SO}(3)$ of $\mathrm{SO}(6)$ are contained in $\mathrm{SO}(3) \times \mathrm{SO}(4) \subset \mathrm{SO}(7)$, which acts with cohomogeneity one on $\mathrm{SO}(7) / \mathrm{G}_{2}$; see 2.4.1. It remains the group $\mathrm{U}(3) \subset \mathrm{SO}(6)$, which leads to a cohomogeneity one action. This may be seen as follows: Instead of $\mathrm{SO}(6) \subset$ $\mathrm{SO}(7)$, consider $\operatorname{Spin}(6) \subset \operatorname{Spin}(7)$. Recall that $\operatorname{Spin}(6) \cong \mathrm{SU}(4)$. Since $\mathrm{U}(3)$ is a maximal subgroup of maximal rank, the corresponding subgroup of $\mathrm{SU}(4)$ is given by Table 5 , ON2, $\S 3$. The only possibility is $\mathrm{S}\left(\mathrm{U}_{1} \times \mathrm{U}_{3}\right) \subset \mathrm{SU}(4)$, thus we have the cohomogeneity one action of $\mathrm{S}\left(\mathrm{U}_{1} \times \mathrm{U}_{3}\right)$ on $\mathrm{S}^{7}=\operatorname{Spin}(7) / \mathrm{G}_{2}$.

5.a) See 4.a).

5.b) The group $\mathrm{SO}(5)$ acts transitively on $\mathrm{SO}(7) / \mathrm{G}_{2}$. The maximal connected subgroups of $\mathrm{SO}(5)$ are: $\mathrm{SO}(4), \mathrm{SO}(2) \times \mathrm{SO}(3)$ and a subgroup given by the 5 dimensional irreducible representation of $\mathrm{A}_{1}$. The first group is contained in the subgroup $\mathrm{SO}(3) \times \mathrm{SO}(4) \subset \mathrm{SO}(7)$, which acts with cohomogeneity one; see 2.4.1 The other two groups may be excluded because their dimensions are too low.

6.a) The maximal connected subgroups of $\mathrm{SO}(7)$ of dimension $\geq 6$ are:

$$
\mathrm{SO}(6), \quad \mathrm{SO}(5) \times \mathrm{SO}(2), \quad \mathrm{SO}(4) \times \mathrm{SO}(3), \quad \mathrm{G}_{2} .
$$

The first two subgroups act transitively on $\mathrm{SO}(8) / \mathrm{Spin}(7)$, they were already examined in 2.a) and 3.a) in the guise of the transitive actions of $\mathrm{SU}(4)$ and $\mathrm{Sp}(2) \cdot \mathrm{U}(1)$ on $\mathrm{S}^{7}$. The third group is contained in $\mathrm{SO}(4) \times \mathrm{SO}(4) \subset \mathrm{SO}(8)$, which acts 
with cohomogeneity one, see 2.4.1 Finally, $\mathrm{G}_{2}$ acts with cohomogeneity one on $\mathrm{SO}(8) / \operatorname{Spin}(7)$, but we may assume $\mathrm{G}_{2} \subset \operatorname{Spin}(7)$ by conjugation.

6.b) If $G=\operatorname{Spin}(8)$ and $H$ and $K$ are two subgroups isomorphic to $\operatorname{Spin}(7)$, there is the maximal connected subgroup $\Delta \operatorname{Spin}(7) \subset \operatorname{Spin}(7) \times \operatorname{Spin}(7) \cong \mathrm{H} \times \mathrm{K}$, but the dimension of this group is too low for a hyperpolar action.

7.a) Among the maximal connected subgroups of $\mathrm{SO}(15)$, the irreducible ones may be excluded by a dimension condition and the reducible subgroups are contained in reducible symmetric subgroups of $\mathrm{SO}(16)$.

7.b) The subgroups of $\mathrm{SO}(16)$ which act hyperpolarly, i.e. with cohomogeneity one on $\mathrm{S}^{15}$ are well-known; see DADOK. We have now completed the classification of the hyperpolar actions on the simple compact Lie groups and thus also on the irreducible symmetric spaces of the compact type; see Proposition 1.10

\subsection{Proof of Theorem A.}

Proof. Let $U \subset G \times G$ be a closed connected subgroup such that the $U$-action on $G$ is hyperpolar and non-transitive. By Theorem 1.8 and the classification of the simple compact Lie groups, we may assume that $G$ is one of the following: $\mathrm{SU}(\mathrm{n})$ $(n \geq 2), \operatorname{Sp}(\mathrm{n})(n \geq 2)$, Spin(n) $(n \geq 7)$ or a simply connected exceptional Lie group $\mathrm{E}_{6}, \mathrm{E}_{7}, \mathrm{E}_{8}, \mathrm{~F}_{4}, \mathrm{G}_{2}$, since the hyperpolarity of the $U$-action on $G$ depends only on the Lie algebra of $U$.

By Theorem 1.11, we have that $U$ is contained in a maximal connected nontransitive subgroup $U^{\prime} \subset G \times G$ such that the $U$-action on $G$ and the $U^{\prime}$-action on $G$ are orbit equivalent.

It now follows from Theorem 2.1 that one of the following is true.

- $U^{\prime}=\{(g, \sigma(g)) \mid g \in G\}$ for some automorphism $\sigma$ of $G$, i.e. the $U^{\prime}$-action on $G$ is a $\sigma$-action.

- $U^{\prime}=H \times K$, where $H$ and $K$ are maximal connected subgroups of $G \times G$. As far as $H \times K$ is not excluded by necessary conditions on the dimensions of groups that act hyperpolarly, see 2.2 the actions of such groups have been examined case by case in 2.322.4.4 in order to determine if they are hyperpolar or not.

- $U^{\prime}$ is a maximal connected subgroup of $H \times K$, where $H \times K$ acts transitively on $G$. These $U^{\prime}$-actions on $G$ are examined in 2.4.5.

By these steps, we have found all local conjugacy classes of hyperpolar actions of maximal connected non-transitive subgroups $U^{\prime} \subset G \times G$ on simple compact Lie groups $G$. It turns out that all hyperpolar actions that are not Hermann or $\sigma$-actions are as described in parts ii), iii), iv) and v) of the theorem, i.e. they are cohomogeneity one actions.

\section{The Classification of Cohomogeneity One Actions}

In this section, we will complete the classification of cohomogeneity one actions on compact irreducible symmetric spaces. Cohomogeneity one actions on symmetric spaces are hyperpolar, thus by the classification of hyperpolar actions obtained in the last section, all that remains to do is to determine which Hermann actions and which $\sigma$-actions are of cohomogeneity one. We do this by computing the cohomogeneities of all Hermann actions and $\sigma$-actions on the simple compact Lie groups. 
3.1. Hermann Actions. To compute the cohomogeneities of the Hermann actions on the simple compact Lie groups, we use the following proposition, which shows that the slice representation of a Hermann action is equivalent to an isotropy representation of a symmetric space.

Proposition 3.1. Let $G$ be a compact connected Lie group. Let $\sigma$ and $\tau$ be involutions of $G$ and let $H=G^{\sigma}$ and $K=G^{\tau}$ be their fixed point groups. Let $G^{\sigma \tau}$ be the fixed point set of $\sigma \circ \tau$. Then $\left(G^{\sigma \tau}, H \cap K\right)$ is a symmetric pair, the isotropy representation of the corresponding symmetric space is equivalent to the slice representation of the $H \times K$-action at $e$ and the cohomogeneity of the action of $H \times K$ on $G$ is equal to $\mathrm{rk}\left(\mathrm{G}^{\sigma \tau} /(\mathrm{H} \cap \mathrm{K})\right)$.

Proof. Restricted to $G^{\sigma \tau}$, the two involutions $\sigma$ and $\tau$ agree and $H \cap K$ is the fixed point group of $\left.\sigma\right|_{G^{\sigma \tau}}=\left.\tau\right|_{G^{\sigma \tau}}$. We have the Cartan decomposition $\mathfrak{g}^{\sigma \tau}=$ $\left(\mathfrak{h}^{\perp} \cap \mathfrak{k}^{\perp}\right) \oplus(\mathfrak{h} \cap \mathfrak{k})$. From this, it follows that the isotropy representation of $H \cap K$ on $\mathfrak{h}^{\perp} \cap \mathfrak{k}^{\perp}$ is equivalent to the slice representation of the $H \times K$-action at $e$, see 2.3 .

We proceed as follows. For every simple compact Lie group $G$, we take all pairs $(H, K)$ of connected symmetric subgroups and compute the cohomogeneity of the $H \times K$-action on $G$. By Proposition 1.13, we may restrict ourselves to consider only one representative of every conjugacy class of $H \times K$ in $G \times G$. We remark that for $\alpha \in \operatorname{Aut}(\mathrm{G})$ we have $\alpha\left(G^{\sigma}\right)=G^{\alpha \sigma \alpha^{-1}}$. By Proposition 1.4 and Theorem $6.1 \mathrm{in}[\mathrm{HE}$, Chapter X, any two isomorphic symmetric subgroups of $\tilde{G}$ are mapped to each other by an automorphism of $\tilde{G}$. (By $\tilde{G}$ we denote the universal cover of G.)

3.1.1. Hermann Actions on the Classical Groups. The cohomogeneities of the Hermann actions on the classical groups can be determined by computing the slice representation of the isotropy group at $e$, using Proposition 3.1 This is a straightforward calculation and we will only state the results in the theorem below. The group $G=\mathrm{SO}(8)$ will be considered separately, because there are more Hermann actions on it, arising from the triality automorphisms of $\operatorname{Spin}(8)$.

Since we excluded the groups locally isomorphic to Spin(8), we have that either Aut $(\mathrm{G})$ is connected or it has two connected components. In the first case all automorphisms are inner and in the latter case for every $\sigma \in \operatorname{Aut}(G) \backslash \operatorname{Inn}(G)$ there is $i_{g} \in \operatorname{Inn}(\mathrm{G})$ such that $\sigma=\sigma_{0} \circ i_{g}$, where $\sigma_{0}$ is an involutive outer automorphism. Thus there are either one or two conjugacy classes of every (isomorphism type of) connected symmetric subgroup of the classical groups except $\mathrm{SO}(8)$. The involutions of the classical groups may be taken from [HE], Chapter X, §2. It turns out that the only case where there are two conjugacy classes of a connected symmetric subgroup is $\mathrm{U}(2 \mathrm{n}) \subset \mathrm{SO}(4 \mathrm{n})$. This may be seen as follows:

The symmetric subgroups of SU(n) which are not fixed point sets of an outer automorphism, i.e. $\mathrm{S}\left(\mathrm{U}_{k} \times \mathrm{U}_{n-k}\right)$, are obviously invariant under complex conjugation, which is an outer automorphism of $\mathrm{SU}(\mathrm{n}), \mathrm{n}>2$.

In $\mathrm{SO}(2 \mathrm{n}), n$ odd, conjugation by the matrix $\operatorname{diag}(+1,-1,+1,-1, \ldots,+1,-1)$ is an outer automorphism, under which the subgroups $\mathrm{SO}(\mathrm{k}) \times \mathrm{SO}(2 \mathrm{n}-\mathrm{k})$ and $\mathrm{U}(\mathrm{n})$ are invariant. (The automorphism of $\mathrm{U}(\mathrm{n})$ that is induced by this map is just complex conjugation of matrices.)

On $\mathrm{SO}(4 \mathrm{n})$, define the outer automorphism $\alpha$ to be conjugation by the matrix $\operatorname{diag}(-1,1, \ldots, 1)$. Clearly, the subgroups $\mathrm{SO}(\mathrm{k}) \times \mathrm{SO}(4 \mathrm{n}-\mathrm{k})$ are invariant under 
$\alpha$. An explicit calculation shows that the cohomogeneity of the $\alpha(\mathrm{U}(2 \mathrm{n}))$-action on $\mathrm{SO}(4 \mathrm{n}) / \mathrm{U}(2 \mathrm{n})$ is one less than the rank of the symmetric space $\mathrm{SO}(4 \mathrm{n}) / \mathrm{U}(2 \mathrm{n})$ (see the following theorem). Therefore $\alpha(\mathrm{U}(2 \mathrm{n}))$ cannot be conjugate to $\mathrm{U}(2 \mathrm{n})$, since it is well-known that on a symmetric space the cohomogeneity of the isotropy action is equal to the rank.

Theorem 3.2. Let $G$ be a classical compact Lie group, $G \neq \mathrm{SO}(8)$. Let $H$ and $K$ be connected symmetric subgroups of $G$ that are not conjugate. The cohomogeneity of the $H \times K$-action on $G$ is given by Table 5 , where $\alpha \in \operatorname{Aut}(\mathrm{SO}(2 \mathrm{n})) \backslash \operatorname{Inn}(\mathrm{SO}(2 \mathrm{n}))$.

\begin{tabular}{|c|c|c|c|}
\hline$H$ & $G$ & $K$ & Cohomogeneity \\
\hline \hline $\mathrm{SO}(2 \mathrm{n})$ & $\mathrm{SU}(2 \mathrm{n})$ & $\mathrm{Sp}(\mathrm{n})$ & $n-1$ \\
\hline $\mathrm{SO}(\mathrm{p}+\mathrm{q})$ & $\mathrm{SU}(\mathrm{p}+\mathrm{q})$ & $\mathrm{S}\left(\mathrm{U}_{p} \times \mathrm{U}_{q}\right)$ & $\min (p, q)$ \\
\hline $\mathrm{S}\left(\mathrm{U}_{\mathrm{k}} \times \mathrm{U}_{2 \mathrm{n}-\mathrm{k}}\right)$ & $\mathrm{SU}(2 \mathrm{n})$ & $\mathrm{Sp}(\mathrm{n})$ & $\min \left(\left\lfloor\frac{k}{2}\right\rfloor\left\lfloor n-\frac{k}{2}\right\rfloor\right)$ \\
\hline $\mathrm{S}\left(\mathrm{U}_{\mathrm{a}+\mathrm{b}} \times \mathrm{U}_{\mathrm{c}}\right)$ & $\mathrm{SU}(\mathrm{a}+\mathrm{b}+\mathrm{c})$ & $\mathrm{S}\left(\mathrm{U}_{\mathrm{a}} \times \mathrm{U}_{\mathrm{b}+\mathrm{c}}\right)$ & $\min (a, c)$ \\
\hline $\mathrm{SO}(\mathrm{a}+\mathrm{b}) \times \mathrm{SO}(\mathrm{c})$ & $\mathrm{SO}(\mathrm{a}+\mathrm{b}+\mathrm{c})$ & $\mathrm{SO}(\mathrm{a}) \times \mathrm{SO}(\mathrm{b}+\mathrm{c})$ & $\min (a, c)$ \\
\hline $\mathrm{Sp}(\mathrm{p}) \times \mathrm{Sp}(\mathrm{q})$ & $\mathrm{Sp}(\mathrm{p}+\mathrm{q})$ & $\mathrm{U}(\mathrm{p}+\mathrm{q})$ & $\min (p, q)$ \\
\hline $\mathrm{Sp}(\mathrm{a}+\mathrm{b}) \times \mathrm{Sp}(\mathrm{c})$ & $\mathrm{Sp}(\mathrm{a}+\mathrm{b}+\mathrm{c})$ & $\mathrm{Sp}(\mathrm{a}) \times \mathrm{Sp}(\mathrm{b}+\mathrm{c})$ & $\min (a, c)$ \\
\hline $\mathrm{SO}(\mathrm{k}) \times \mathrm{SO}(2 \mathrm{n}-\mathrm{k})$ & $\mathrm{SO}(2 \mathrm{n})$ & $\mathrm{U}(\mathrm{n})$ & $\min \left(\left\lfloor\frac{k}{2}\right\rfloor\left\lfloor n-\frac{k}{2}\right\rfloor\right)$ \\
\hline $\mathrm{U}(\mathrm{n})$ & $\mathrm{SO}(2 \mathrm{n})$ & $\alpha(\mathrm{U}(\mathrm{n}))$ & {$\left[\frac{n-1}{2}\right]$} \\
\hline
\end{tabular}

TABLE 5. Hermann actions on the classical groups

Remark. The actions $G=\mathrm{SO}(2 \mathrm{n}), H=\mathrm{U}(\mathrm{n}), K=\alpha(\mathrm{U}(\mathrm{n}))$, which are of cohomogeneity one if $n=3$ or $n=4$ do not occur explicitly in Theorem $\mathrm{B}$, because in the case $n=3$ the subgroups $H$ and $K$ are conjugate and in the case $n=4$ the action is locally conjugate to the action $G=\mathrm{SO}(8), H=\mathrm{U}(4), K=\mathrm{SO}(2) \times \mathrm{SO}(6)$ (see Prop. [3.3).

3.1.2. Hermann Actions on $\mathrm{SO}(8)$. Let $G$ be a group and $H \subset G$ be a subgroup. The group of outer automorphisms $\operatorname{Out}(\mathrm{G})=\operatorname{Aut}(\mathrm{G}) / \operatorname{Inn}(\mathrm{G})$ acts on the set of conjugacy classes of $H$ in $G$. In the case $G=\operatorname{Spin}(8), \operatorname{Out}(G) \cong \operatorname{Sym}(3)$, i.e. the group of outer automorphisms of $\operatorname{Spin}(8)$ is isomorphic to the permutation group on three letters, which is the automorphism group of the Dynkin diagram $\mathrm{D}_{4}$.

As mentioned in 3.1, isomorphic connected symmetric subgroups of $\tilde{G}$ are mapped to each other by automorphisms of $\tilde{G}$. Thus if $H \subset \tilde{G}$ is a connected symmetric subgroup, $\operatorname{Out}(\tilde{\mathrm{G}})$ acts transitively on the set of conjugacy classes of $H$.

Proposition 3.3. The four local isomorphism types of symmetric subgroups of $\mathrm{SO}(8)$ are given by the rows of the following table, where also for each conjugacy class of connected symmetric subgroups one representative is given.

\begin{tabular}{|l|l|}
\hline 1. & $\mathrm{SO}(7), \mathrm{Spin}^{+}(7), \mathrm{Spin}^{-}(7)$ \\
\hline 2. & $\mathrm{SO}(2) \times \mathrm{SO}(6), \mathrm{U}(4), \alpha(\mathrm{U}(4))$ \\
\hline 3. & $\mathrm{SO}(3) \times \mathrm{SO}(5), \mathrm{Sp}(1) \otimes \mathrm{Sp}(2), \mathrm{Sp}(2) \otimes \mathrm{Sp}(1)$ \\
\hline 4. & $\mathrm{SO}(4) \times \mathrm{SO}(4)$ \\
\hline
\end{tabular}

Here, $\alpha$ denotes conjugation by the matrix $\operatorname{diag}(-1,1,1,1,1,1,1,1)$. 
Proof. We have that $\operatorname{Out}(\operatorname{Spin}(8)) \cong \operatorname{Sym}(3)$ acts transitively on the set of conjugacy classes of connected symmetric subgroups of $\mathrm{SO}(8)$. Further, the outer involution $\alpha$ leaves the reducible subgroups $\mathrm{SO}(\mathrm{k}) \times \mathrm{SO}(8-\mathrm{k})$ invariant, i.e. the corresponding element in $\operatorname{Out}(\operatorname{Spin}(8))$ of order two is contained in the isotropy group of this conjugacy class; from this it follows that we have either three or one conjugacy class for every local isomorphism type of connected symmetric subgroups of $\mathrm{SO}(8)$.

It is well-known that there are three conjugacy classes of groups locally isomorphic to $\mathrm{SO}(7)$; see [ON2], Chapter 1, $§ 3$, Example 9.

Since No. 2 and No. 4 are subgroups of maximal rank, their conjugacy classes may be taken from ON2, Theorem 16, $\S 3$.

Finally, it can be directly verified that the half spin representations of $\mathfrak{s o}(8)$ map the subalgebra $\mathfrak{s o}(3)+\mathfrak{s o}(5)$ to $\mathfrak{s p}(1) \otimes \mathfrak{s p}(2)$ or $\mathfrak{s p}(2) \otimes \mathfrak{s p}(1)$.

Using this proposition, we can determine the Hermann actions on $\mathrm{SO}(8)$ up to local conjugacy.

Theorem 3.4. Let $H, K \in \mathrm{SO}(8)$ be two non-conjugate connected symmetric subgroups. The cohomogeneity of the $H \times K$-action on $G$ may be taken from Table 5 or Table 6.

\begin{tabular}{|c|c|c|}
\hline$H$ & $K$ & Cohomogeneity \\
\hline \hline $\mathrm{Spin}(7)$ & $\mathrm{SO}(7)$ & 0 \\
\hline $\mathrm{Sp}(1) \otimes \mathrm{Sp}(2)$ & $\mathrm{SO}(7)$ & 0 \\
\hline $\mathrm{Sp}(1) \otimes \mathrm{Sp}(2)$ & $\mathrm{SO}(3) \times \mathrm{SO}(5)$ & 2 \\
\hline
\end{tabular}

TABle 6. Additional Hermann actions on $\mathrm{SO}(8)$

Proof. The transitivity of the first and the second action are well-known facts.

In 2.3.2, Case 3.2.1 $(n=3)$ it is shown that a slice representation of the $\operatorname{Sp}(1) \otimes$ $\mathrm{Sp}(2)$-action on $\mathrm{G}_{3}\left(\mathbb{R}^{8}\right)$ is equivalent to the isotropy representation of the ranktwo symmetric space $\mathrm{G}_{2} / \mathrm{SO}(4)$. This proves that the action is of cohomogeneity two.

3.1.3. Hermann Actions on the Exceptional Groups. Our method to compute the cohomogeneity of a Hermann action on an exceptional group relies on a result of Conlon.

Proposition 3.5. Let $G$ be a compact Lie group. Let $\sigma$ and $\tau$ be two different, commuting involutions of $G$ and let $H=G^{\sigma}, K=G^{\tau}$. In this case, we have the following diagram, where all six arrows denote inclusions of symmetric subgroups:

$G$

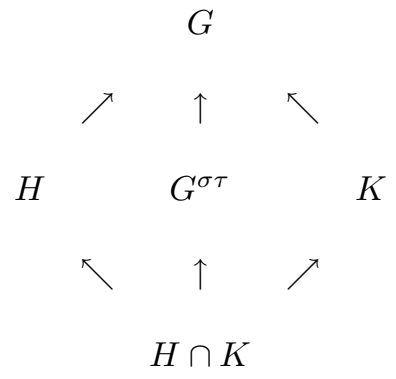


Proof. By the hypothesis, $(G, H),(G, K)$ are symmetric pairs. The automorphism $\sigma \circ \tau$ is an involution of $G$, because $\sigma$ and $\tau$ are different, commuting involutions: $\sigma \circ \tau=\sigma \circ \tau^{-1} \neq 1$ and $(\sigma \circ \tau)^{2}=\sigma^{2} \circ \tau^{2}=1$. Therefore $\left(G, G^{\sigma \tau}\right)$ is a symmetric pair. The pair $\left(G^{\sigma \tau}, H \cap K\right)$ is symmetric by Proposition 3.1. Finally, Proposition 3.1 may be applied to the Hermann actions of $H \times G^{\sigma \tau}$ and $K \times G^{\sigma \tau}$ on $G$. Obviously, $H \cap G^{\sigma \tau}=K \cap G^{\sigma \tau}=H \cap K$. Hence, $(H, H \cap K)$ and $(K, H \cap K)$ are symmetric pairs as well.

We remark that from such a diagram, the cohomogeneity of all three Hermann actions involved can be read off by Proposition 3.1. It remains to decide in which cases we may assume that two given involutions $\sigma$ and $\tau$ commute, i.e. if there is an inner automorphism $i_{g}$ of $G$, such that $\sigma$ and $i_{g} \circ \tau \circ i_{g}^{-1}$ commute. This problem was solved by $\mathrm{CO} 2$ for the simple compact Lie groups. From the results of [Co2], it can be seen that for the exceptional Lie groups such an $i_{g}$ always exists, i.e. we can apply Proposition [3.5 to compute the cohomogeneity of all Hermann actions on the exceptional compact Lie groups.

We proceed as follows. Let $H$ and $K$ be the connected components of the fixed point sets of the involutions $\sigma$ and $\tau$ of $G$, respectively. By [Co2], we may assume that $\sigma$ and $\tau$ commute. Thus, by Proposition 3.5 we have that $H \cap K$ is a symmetric subgroup of both $H$ and $K$, and that $\left(G, G^{\sigma \tau}\right)$ and $\left(G^{\sigma \tau}, H \cap K\right)$ are symmetric pairs. By the classification of the symmetric spaces, this leaves only a small number of possibilities for the local isomorphism types of $G^{\sigma \tau}$ and $H \cap K$. The cohomogeneity is then given by the rank of the symmetric space $G^{\sigma \tau} /(H \cap$ $K)$. In the cases where there remain several possible values for $\operatorname{rk}\left(\mathrm{G}^{\sigma \tau} /(\mathrm{H} \cap \mathrm{K})\right)$, some possibilities may be excluded, either using the fact that the cohomogeneity of the $H \times K$-action on $G$ is smaller or equal to $\min (\operatorname{rk}(\mathrm{G} / \mathrm{H}), \mathrm{rk}(\mathrm{G} / \mathrm{K}))$, or by the necessary condition for the dimensions of $G^{\sigma \tau}$ and $H \cap K$ given by the equality

$$
\operatorname{dim} G^{\sigma \tau}-2 \operatorname{dim} H \cap K=\operatorname{dim} G-\operatorname{dim} H \times K,
$$

which follows from Proposition 3.1. By this method, the cohomogeneities of all Hermann actions on the exceptional groups can be determined.

Note that there are no non-trivial transitive actions on the exceptional Lie groups, i.e. if $G=H \cdot K$, then either $H=G$ or $K=G$; see 2.4.5.

Hermann Actions on $\mathrm{E}_{6}$. In $G=\mathrm{E}_{6}$, any two isomorphic symmetric subgroups are conjugate, i.e. are mapped onto each other by an inner automorphism of $\mathrm{E}_{6}$. This can be seen as follows: Let $G^{\sigma}$ be the fixed point set of the involution $\sigma$. As mentioned above, we know that any other isomorphic symmetric subgroup $K$ differs from $G^{\sigma}$ by a (possibly outer) automorphism $\alpha$, i.e. $K=\alpha\left(\mathrm{G}^{\sigma}\right)=\mathrm{G}^{\alpha \sigma \alpha^{-1}}$. Since the diagram automorphism of $\mathrm{E}_{6}$ is also an involution, we may assume by the result of [CO2], that $\sigma$ and $\alpha$ commute and hence that, after conjugation, $G^{\alpha \sigma \alpha^{-1}}=\mathrm{G}^{\sigma}$ (see also [DYN1], p. 146).

We start with the action of $\operatorname{Sp}(4) /\{ \pm 1\} \times \mathrm{F}_{4}$ on $G=\mathrm{E}_{6}$. In this case the involutions corresponding to $H$ and $K$ are both outer automorphisms. Hence their composition is an inner automorphism and its fixed point group has full rank; see [WOLF, Theorem 8.6.7. Thus the connected component of $G^{\sigma \tau}$ is either $\mathrm{SU}(6) \cdot \mathrm{SU}(2)$ or $\operatorname{Spin}(10) \cdot \mathrm{SO}(2)$. Now, because $\left(\mathrm{F}_{4}, \mathrm{H} \cap \mathrm{K}\right)$ is a symmetric pair, $(H \cap K)_{0}$ is either conjugate to $\mathrm{Sp}(3) \cdot \mathrm{Sp}(1)$ or $\operatorname{Spin}(9)$. The only remaining possibility is as in the following diagram, from which the cohomogeneity of the two other Hermann actions 
may be read off as well:

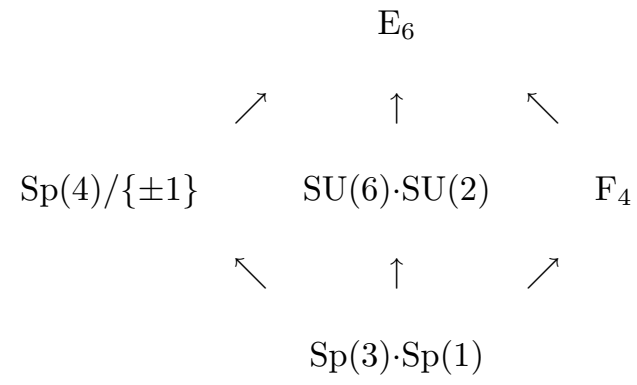

In particular, we have found the cohomogeneity one action of $\mathrm{SU}(6) \cdot \mathrm{SU}(2) \times \mathrm{F}_{4}$ on $\mathrm{E}_{6}$.

Now let $H=\mathrm{SU}(6) \cdot \mathrm{SU}(2)$ and $K=\operatorname{Spin}(10) \cdot \mathrm{SO}(2)$. We list the connected symmetric subgroups of $\mathrm{SU}(6)$, together with their Lie algebra types:

\begin{tabular}{|c|c|c|c|c|}
\hline $\mathrm{S}\left(\mathrm{U}_{1} \times \mathrm{U}_{5}\right)$ & $\mathrm{S}\left(\mathrm{U}_{2} \times \mathrm{U}_{4}\right)$ & $\mathrm{S}\left(\mathrm{U}_{3} \times \mathrm{U}_{3}\right)$ & $\mathrm{SO}(6)$ & $\mathrm{Sp}(3)$ \\
\hline $\mathfrak{a}_{4}+\mathbb{R}$ & $\mathfrak{a}_{1}+\mathfrak{a}_{3}+\mathbb{R}$ & $\mathfrak{a}_{2}+\mathfrak{a}_{2}+\mathbb{R}$ & $\mathfrak{a}_{3}$ & $\mathfrak{c}_{3}$ \\
\hline
\end{tabular}

These are the connected symmetric subgroups of $\mathrm{SO}(10)$ :

\begin{tabular}{|c|c|c|}
\hline $\mathrm{SO}(9)$ & $\mathrm{SO}(8) \times \mathrm{SO}(2)$ & $\mathrm{SO}(7) \times \mathrm{SO}(3)$ \\
\hline $\mathfrak{b}_{4}$ & $\mathfrak{d}_{4}+\mathbb{R}$ & $\mathfrak{b}_{3}+\mathfrak{a}_{1}$ \\
\hline
\end{tabular}

\begin{tabular}{|c|c|c|}
\hline $\mathrm{SO}(6) \times \mathrm{SO}(4)$ & $\mathrm{SO}(5) \times \mathrm{SO}(5)$ & $\mathrm{U}(5)$ \\
\hline $\mathfrak{a}_{3}+\mathfrak{a}_{1}+\mathfrak{a}_{1}$ & $\mathfrak{b}_{2}+\mathfrak{b}_{2}$ & $\mathfrak{a}_{4}+\mathbb{R}$ \\
\hline
\end{tabular}

One possibility for $\mathfrak{h} \cap \mathfrak{k}$ is $\mathfrak{s u}(5)+\mathbb{R}+\mathbb{R}$. Since the two involutions are inner, $G_{0}^{\sigma \tau}$ is a symmetric subgroup of full rank; see [WOLF, Theorem 8.6.7. Thus we have either $\mathrm{SU}(6) \cdot \mathrm{SU}(2)$ or $\mathrm{Spin}(10) \cdot \mathrm{SO}(2)$. In both cases the resulting cohomogeneity is 2 . The other possibility $\mathfrak{h} \cap \mathfrak{k}=\mathfrak{a}_{3}+\mathfrak{a}_{1}+\mathfrak{a}_{1}+\mathbb{R}$ also leads to cohomogeneity 2 .

Now assume $H=\mathrm{F}_{4}$ and $K=\operatorname{Spin}(10) \cdot \mathrm{SO}(2)$. The only possibility for $(H \cap K)_{0}$ is $\operatorname{Spin}(9)$. The Lie algebra $\mathfrak{g}^{\sigma \tau}$ is the fixed point set of an outer automorphism of $\mathfrak{e}_{6}$, thus isomorphic to either $\mathfrak{c}_{4}$ or $\mathfrak{f}_{4}$. But $\mathfrak{s p i n}(9) \cong \mathfrak{b}_{4}$ is not a subalgebra of $\mathfrak{c}_{4}$. Thus $\operatorname{rk}\left(\mathrm{G}^{\sigma \tau} /(\mathrm{H} \cap \mathrm{K})\right)=\operatorname{rk}\left(\mathrm{F}_{4} / \operatorname{Spin}(9)\right)=1$ and we have found another cohomogeneity one action.

Finally, let $H=\mathrm{Sp}(4) /\{ \pm 1\}$ and $K=\operatorname{Spin}(10) \cdot \mathrm{SO}(2)$. The only possibility is $\operatorname{rk}\left(\mathrm{G}^{\sigma \tau} /(\mathrm{H} \cap \mathrm{K})\right)=\operatorname{rk}(\mathrm{Sp}(4) /[\mathrm{Sp}(2) \times \mathrm{Sp}(2)])=2$.

Hermann Actions on $\mathrm{E}_{7}$ and $\mathrm{E}_{8}$. We do not give proofs here, since they are very similar to the $\mathrm{E}_{6}$ case.

Hermann Actions on $\mathrm{F}_{4}$ and $\mathrm{G}_{2}$. Since $\mathrm{rk}\left(\mathrm{F}_{4} / \operatorname{Spin}(9)\right)=1$, the action of $\operatorname{Sp}(3)$. $\mathrm{Sp}(1)$ is of cohomogeneity one. Up to conjugacy, there is no Hermann action on $\mathrm{G}_{2}$ besides the action of $\mathrm{SO}(4) \times \mathrm{SO}(4)$.

Theorem 3.6. Let $G$ be an exceptional simple compact Lie group. Let $H$ and $K$ be connected symmetric subgroups of $G$ that are not conjugate. The cohomogeneity of the $H \times K$-action on $G$ is given by Table 7 . 


\begin{tabular}{|c|c|c|c|}
\hline$H$ & $G$ & $K$ & Cohomogeneity \\
\hline \hline $\mathrm{Sp}(4) /\{ \pm 1\}$ & $\mathrm{E}_{6}$ & $\mathrm{SU}(6) \cdot \mathrm{SU}(2)$ & 4 \\
\hline $\mathrm{Sp}(4) /\{ \pm 1\}$ & $\mathrm{E}_{6}$ & $\mathrm{Spin}(10) \cdot \mathrm{SO}(2)$ & 2 \\
\hline $\mathrm{Sp}(4) /\{ \pm 1\}$ & $\mathrm{E}_{6}$ & $\mathrm{~F}_{4}$ & 2 \\
\hline $\mathrm{SU}(6) \cdot \mathrm{SU}(2)$ & $\mathrm{E}_{6}$ & $\mathrm{Spin}(10) \cdot \mathrm{SO}(2)$ & 2 \\
\hline $\mathrm{SU}(6) \cdot \mathrm{SU}(2)$ & $\mathrm{E}_{6}$ & $\mathrm{~F}_{4}$ & 1 \\
\hline $\mathrm{Spin}(10) \cdot \mathrm{SO}(2)$ & $\mathrm{E}_{6}$ & $\mathrm{~F}_{4}$ & 1 \\
\hline $\mathrm{SU}(8) /\{ \pm 1\}$ & $\mathrm{E}_{7}$ & $\mathrm{SO}(12)^{\prime} \cdot \mathrm{SU}(2)$ & 4 \\
\hline $\mathrm{SU}(8) /\{ \pm 1\}$ & $\mathrm{E}_{7}$ & $\mathrm{E}_{6} \cdot \mathrm{SO}(2)$ & 3 \\
\hline $\mathrm{SO}(12)^{\prime} \cdot \mathrm{SU}(2)$ & $\mathrm{E}_{7}$ & $\mathrm{E}_{6} \cdot \mathrm{SO}(2)$ & 2 \\
\hline $\mathrm{SO}^{\prime}(16)$ & $\mathrm{E}_{8}$ & $\mathrm{E}_{7} \cdot \mathrm{SU}(2)$ & 4 \\
\hline $\mathrm{Sp}(3) \cdot \mathrm{Sp}(1)$ & $\mathrm{F}_{4}$ & $\mathrm{Spin}(9)$ & 1 \\
\hline
\end{tabular}

TABLE 7. Hermann actions on the exceptional Lie groups

3.2. $\sigma$-Actions. Finally, we will compute the cohomogeneities of the $\sigma$-actions on the simple compact Lie groups. Let $G$ be a simple compact Lie group and let $\sigma$ be an automorphism of $G$. The action of the group $\Delta^{\sigma} G=\{(g, \sigma(g)) \mid g \in G\}$ on $G$ is called the $\sigma$-action. By Proposition 1.13, the $\sigma$-action is conjugate to the adjoint action of $G$ if $\sigma$ is an inner automorphism of $G$. Therefore, we only have to consider the cases where the automorphism group of $G$ is not connected, i.e.

$$
G=\mathrm{A}_{\mathrm{n}}(\mathrm{n} \geq 2), \mathrm{D}_{\mathrm{n}}(\mathrm{n} \geq 4), \mathrm{E}_{6},
$$

and where $\sigma$ is an outer automorphism. As before, we determine the cohomogeneities of the $\sigma$-actions by computing slice representations. The isotropy group of the identity element $e \in G$ is isomorphic to the fixed point set of $\sigma$ :

$$
\left(\Delta^{\sigma} G\right)_{e}=\left\{(g, g) \mid g \in G^{\sigma}\right\} .
$$

Let $\sigma_{*}: \mathfrak{g} \rightarrow \mathfrak{g}$ be the differential of $\sigma$ at $e$. The tangent space at $e$ to the orbit of $e$ is given by

$$
\left\{X-\sigma_{*}(X) \mid X \in \mathfrak{g}\right\} .
$$

The normal space at $e$ to the orbit of $e$ is $\mathfrak{g}^{\sigma}$, i.e. the fixed point set of $\sigma_{*}$. From (3.4) it follows that the isotropy representation at $e$ is equivalent to the restriction of the adjoint representation of $G$ to $G^{\sigma}$. Hence the slice representation is equivalent to the adjoint representation of $G^{\sigma}$ and, in particular, the cohomogeneity of the $\sigma$-action equals the rank of $G^{\sigma}$.

Since the $\sigma$-actions for two different automorphisms of the same connected component of $\operatorname{Aut}(\mathrm{G})$ are conjugate by Proposition 1.13, we may assume that $\sigma$ is an automorphism of $\tilde{G}$ induced by an automorphism of the Dynkin diagram. In this case we may take the Lie algebras $\mathfrak{g}^{\sigma}$ from Table I, on p. 505 in $[\mathrm{HE}, \mathrm{Ch} . \mathrm{X}, \S 5$.

Theorem 3.7. Let $G$ be a simple compact Lie group and $\sigma$ an outer automorphism of $G$ of finite order. The cohomogeneity of the $\sigma$-action of $G$ is given by Table $\square$.

Together with the results of Section 2, we have now obtained a classification of the cohomogeneity one actions on the simple compact Lie groups, and thus also on the irreducible symmetric spaces of type III. This generalizes the classifications of 


\begin{tabular}{|c|c|c|c|c|}
\hline$G$ & $\mathrm{SU}(\mathrm{n})$ & $\mathrm{SO}(2 \mathrm{n})$ & $\mathrm{E}_{6}$ & $\mathrm{Spin}(8)$ \\
\hline $\operatorname{ord}(\sigma)$ & 2 & 2 & 2 & 3 \\
\hline Cohomogeneity & $\left\lfloor\frac{n}{2}\right\rfloor$ & $n-1$ & 4 & 2 \\
\hline
\end{tabular}

TABLE 8. $\sigma$-Actions

homogeneous hypersurfaces in spheres, complex and quaternionic projective space and the Cayley plane; see [HsL], TAKAGI], [D'ATRI and [IWATA.

\subsection{Proof of Theorem B.}

Proof. By Proposition 1.9, cohomogeneity one actions on $G$ are hyperpolar if $G$ is equipped with a bi-invariant metric.

The local conjugacy classes of hyperpolar actions of maximal connected nontransitive subgroups of $G \times G$ on the simple compact Lie groups $G$ are given by Theorem A. The cohomogeneity one actions that are not locally conjugate to Hermann actions or $\sigma$-actions are described in parts ii), iii), iv) and v) of Theorem A.

The cohomogeneities of the Hermann actions and the $\sigma$-actions on the simple compact Lie groups are given in Theorems 3.2, 3.4, 3.6 and 3.7.

\section{Appendix: Representations of Low Degree}

By the following tables, all irreducible representations of simple compact Lie groups which satisfy the conditions (2.9), (2.10) or (2.11), respectively, are given. The following information is included in the tables: The highest weight $\left(c_{1}, \ldots, c_{n}\right)$ of the representation w.r.t. a basis of fundamental weights. The degree (=dimension) $\operatorname{deg} \varrho$ of the representation. The type of the representation, i.e. if the representation is of real $(\varepsilon(\varrho)=+1)$, complex $(\varepsilon(\varrho)=0)$ or quaternionic $(\varepsilon(\varrho)=-1)$ type. A description of the representation: If the representation is the standard representation of a classical Lie group, then the Lie group, e.g. $\mathrm{SO}(6)$ is given. If the representation is as described in Lemma 2.6. i), ii), or iii), then the type of the corresponding symmetric space, e.g. G, A I, C I (see [HE]) is given.

\begin{tabular}{|l|r|r|l|}
\hline \multicolumn{2}{|c|}{ Lie algebra $\mathrm{A}_{\mathrm{n}}=\mathfrak{s u}(\mathrm{n}+1)$} & \multicolumn{2}{|c|}{$\operatorname{dim} \mathrm{A}_{\mathrm{n}}=\mathrm{n}(\mathrm{n}+2)$} \\
\hline$\left(c_{1}, \ldots, c_{n}\right)$ & $\operatorname{deg} \varrho$ & $\varepsilon(\varrho)$ & Description \\
\hline \hline$(3)$ & 4 & -1 & $\mathrm{G}$ \\
\hline$(4)$ & 5 & +1 & $\mathrm{~A} \mathrm{I}$ \\
\hline$(6)$ & 7 & +1 & \\
\hline$(0,1,0)$ & 6 & +1 & $\mathrm{SO}(6)$ \\
\hline$(0,2,0)$ & 20 & +1 & $\mathrm{~A} \mathrm{I}$ \\
\hline$(0,0,1,0,0)$ & 20 & -1 & $\mathrm{E} \mathrm{II}$ \\
\hline$(0,0,1,0,0,0)$ & 35 & 0 & \\
\hline$(0,0,1,0,0,0,0)$ & 56 & 0 & \\
\hline$(0,0,0,1,0,0,0)$ & 70 & +1 & $\mathrm{E} \mathrm{V}$ \\
\hline$(1,0, \ldots, 0,0)$ & $n+1$ & 0 & $\mathrm{SU}(\mathrm{n}+1)$ \\
\hline$(0,1,0, \ldots, 0)$ & $\frac{1}{2} n(n+1)$ & 0 & $\mathrm{D} \mathrm{III}$ \\
\hline$(2,0, \ldots, 0,0)$ & $\frac{1}{2}(n+1)(n+2)$ & 0 & $\mathrm{C} \mathrm{I}$ \\
\hline$(1,0, \ldots, 0,1)$ & $n(n+2)$ & +1 & adjoint \\
\hline
\end{tabular}




\begin{tabular}{|l|r|r|l|}
\hline \multicolumn{2}{|c|}{ Lie algebra $\mathrm{B}_{\mathrm{n}}=\mathfrak{s o}(2 \mathrm{n}+1)$} & \multicolumn{2}{|c|}{$\operatorname{dim} \mathrm{B}_{\mathrm{n}}=\mathrm{n}(2 \mathrm{n}+1)$} \\
\hline$\left(c_{1}, \ldots, c_{n}\right)$ & $\operatorname{deg} \varrho$ & $\varepsilon(\varrho)$ & Description \\
\hline \hline$(0,1)$ & 4 & -1 & $\mathrm{Sp}(2)$ \\
\hline$(0,0,1)$ & 8 & +1 & $\mathrm{Spin}(7)$ \\
\hline$(0,0,2)$ & 35 & +1 & \\
\hline$(0,0,0,1)$ & 16 & +1 & $\mathrm{~F} \mathrm{II}$ \\
\hline$(0,0,0,0,1)$ & 32 & -1 & spin \\
\hline$(0,0,0,0,0,0,1)$ & 128 & +1 & spin \\
\hline$(0,0,0,0,0,0,0,1)$ & 256 & +1 & spin \\
\hline$(1,0,0, \ldots, 0)$ & $2 n+1$ & +1 & $\mathrm{SO}(2 \mathrm{n}+1)$ \\
\hline$(0,1,0, \ldots, 0)$ & $n(2 n+1)$ & +1 & adjoint \\
\hline$(2,0,0, \ldots, 0)$ & $n(2 n+3)$ & +1 & A I \\
\hline
\end{tabular}

\begin{tabular}{|l|r|r|l|}
\hline \multicolumn{2}{|c|}{ Lie algebra $\mathrm{C}_{\mathrm{n}}=\mathfrak{s p}(\mathrm{n})$} & \multicolumn{2}{|c|}{$\operatorname{dim} \mathrm{C}_{\mathrm{n}}=\mathrm{n}(2 \mathrm{n}+1)$} \\
\hline$\left(c_{1}, \ldots, c_{n}\right)$ & $\operatorname{deg} \varrho$ & $\varepsilon(\varrho)$ & Description \\
\hline \hline$(0,0,1)$ & 14 & -1 & F I \\
\hline$(0,0,0,1)$ & 42 & +1 & E I \\
\hline$(1,0,0, \ldots, 0)$ & $2 n$ & -1 & $\mathrm{Sp}(\mathrm{n})$ \\
\hline$(0,1,0, \ldots, 0)$ & $(n-1)(2 n+1)$ & +1 & A II \\
\hline$(2,0,0, \ldots, 0)$ & $n(2 n+1)$ & +1 & adjoint \\
\hline
\end{tabular}

\begin{tabular}{|l|r|r|l|}
\hline \multicolumn{2}{|c|}{ Lie algebra $\mathrm{D}_{\mathrm{n}}=\mathfrak{s o}(2 \mathrm{n})$} & \multicolumn{2}{|c|}{$\operatorname{dim} \mathrm{D}_{\mathrm{n}}=\mathrm{n}(2 \mathrm{n}-1)$} \\
\hline$\left(c_{1}, \ldots, c_{n}\right)$ & $\operatorname{deg} \varrho$ & $\varepsilon(\varrho)$ & Description \\
\hline \hline$(1,0,1,0)$ & 56 & +1 & \\
\hline$(0,0,0,1,0)$ & 16 & 0 & half-spin, E III \\
\hline$(0,0,0,0,1,0)$ & 32 & -1 & half-spin, E VI \\
\hline$(0,0,0,0,0,1,0)$ & 64 & 0 & half-spin \\
\hline$(0,0,0,0,0,0,1,0)$ & 128 & +1 & half-spin, E VIII \\
\hline$(1,0,0, \ldots, 0)$ & $2 n$ & +1 & $\mathrm{SO}(2 \mathrm{n})$ \\
\hline$(0,1,0, \ldots, 0)$ & $n(2 n-1)$ & +1 & adjoint \\
\hline$(2,0,0, \ldots, 0)$ & $(n+1)(2 n-1)$ & +1 & A I \\
\hline
\end{tabular}

\begin{tabular}{|l|r|r|l|}
\hline \multicolumn{2}{|c|}{ Lie algebra $\mathrm{E}_{6}$} & \multicolumn{2}{|c|}{$\operatorname{dim} \mathrm{E}_{6}=78$} \\
\hline$\left(c_{1}, \ldots, c_{6}\right)$ & $\operatorname{deg} \varrho$ & $\varepsilon(\varrho)$ & Description \\
\hline \hline$(1,0,0,0,0,0)$ & 27 & 0 & E VII \\
\hline$(0,0,0,0,0,1)$ & 78 & +1 & adjoint \\
\hline
\end{tabular}

\begin{tabular}{|l|r|r|l|}
\hline \multicolumn{2}{|c|}{ Lie algebra $\mathrm{E}_{7}$} & \multicolumn{2}{|c|}{$\operatorname{dim} \mathrm{E}_{7}=133$} \\
\hline$\left(c_{1}, \ldots, c_{7}\right)$ & $\operatorname{deg} \varrho$ & $\varepsilon(\varrho)$ & Description \\
\hline \hline$(1,0,0,0,0,0,0)$ & 56 & -1 & E IX \\
\hline$(0,0,0,0,0,1,0)$ & 133 & +1 & adjoint \\
\hline
\end{tabular}

\begin{tabular}{|l|r|r|l|}
\hline \multicolumn{2}{|c|}{ Lie algebra $\mathrm{E}_{8}$} & \multicolumn{2}{|c|}{$\operatorname{dim} \mathrm{E}_{8}=248$} \\
\hline$\left(c_{1}, \ldots, c_{8}\right)$ & $\operatorname{deg} \varrho$ & $\varepsilon(\varrho)$ & Description \\
\hline \hline$(1,0,0,0,0,0,0,0)$ & 248 & +1 & adjoint \\
\hline
\end{tabular}




\begin{tabular}{|l|r|r|l|}
\hline \multicolumn{2}{|c|}{ Lie algebra $\mathrm{F}_{4}$} & \multicolumn{2}{|c|}{$\operatorname{dim} \mathrm{F}_{4}=52$} \\
\hline$\left(c_{1}, \ldots, c_{4}\right)$ & $\operatorname{deg} \varrho$ & $\varepsilon(\varrho)$ & Description \\
\hline \hline$(1,0,0,0)$ & 26 & +1 & E IV \\
\hline$(0,0,0,1)$ & 52 & +1 & adjoint \\
\hline
\end{tabular}

\begin{tabular}{|l|r|r|l|}
\hline \multicolumn{3}{|c|}{ Lie algebra $\mathrm{G}_{2}$} & \multicolumn{2}{l|}{$\operatorname{dim} \mathrm{G}_{2}=14$} \\
\hline$\left(c_{1}, c_{2}\right)$ & $\operatorname{deg} \varrho$ & $\varepsilon(\varrho)$ & Description \\
\hline \hline$(1,0)$ & 7 & +1 & Aut(Ca) \\
\hline$(0,1)$ & 14 & +1 & adjoint \\
\hline$(2,0)$ & 27 & +1 & \\
\hline
\end{tabular}

\section{REFERENCES}

[Besse] Besse A. L.: Einstein Manifolds, Springer-Verlag, (1987) MR 88f:53087

[BMP] Bremner M.R., Moody R.V., Patera J.: Tables of dominant weight multiplicities for representations of simple Lie algebras, Dekker, New York (1985) MR 86f:17002

[Co1] Conlon L.: The topology of certain spaces of paths on a compact symmetric space, Trans. Amer. Math. Soc. 112, 228-248 (1964) MR 29:631

[Co2] Conlon L.: Remarks on commuting involutions, Proc. Amer. Math. Soc. 22, 255-257 (1969) MR 39:4873

[DADOK] Dadok J.: Polar coordinates induced by actions of compact Lie groups, Trans. Amer. Math. Soc. 288, 125-137 (1985) MR 86k:22019

[D'Atri] Datri J.E.: Certain isoparametric families of hypersurfaces in symmetric spaces, J. Differential Geom. 14, 21-40 (1979) MR 81g:53041

[Dyn1] Dynkin E.B.: Semisimple subalgebras of the semisimple Lie algebras, Amer. Math. Soc. Transl. Ser. 2, 6, 111-244 (1952)

[Dyn2] Dynkin E.B.: The maximal subgroups of the classical groups, Amer. Math. Soc. Transl. Ser. 2, 6, 245-378 (1952)

[HL] Heintze E., Liu X.: A splitting theorem for isoparametric submanifolds in Hilbert space, J. Differential Geom. 45, 319-335 (1997) MR 98c:58006

[HPTT1] Heintze E., Palais R., Terng C.-L., Thorbergsson G.: Hyperpolar actions on symmetric spaces, Geometry, topology and physics for Raoul Bott, (S.-T. Yau, ed.), International Press, Cambridge, (1995) MR 96i:53052

[HPTT2] Heintze E., Palais R., Terng C.-L., Thorbergsson G.: Hyperpolar actions and $k$-flat homogeneous spaces, J. Reine Angew. Math. 454, 163-179 (1994) MR 96b:53062

[HE] Helgason S.: Differential geometry, Lie groups and symmetric spaces, Academic Press (1978) MR 80k:53081

[HR] Hermann R.: Variational completeness for compact symmetric spaces, Proc. Amer. Math. Soc. 11, 544-546 (1960) MR 23:A1748

[HsL] Hsiang W.Y., Lawson H.B.: Minimal submanifolds of low cohomogeneity, J. Differential Geom. 5, 1-38 (1971) MR 45:7645

[HsHs] Hsiang W.C., Hsiang W.Y.: Differentiable actions of compact connected classical groups II, Ann. of Math. (2) 92, 189-223 (1970) MR 42:420

[Iwata] Iwata, K.: Compact transformation groups on rational cohomology Cayley projective planes, Tôhoku Math. J., 33, 429-422 (1981) MR 83h:57047

[JÄNICH] Jänich K.: Differenzierbare G-Mannigfaltigkeiten, Lecture Notes in Math. 59, SpringerVerlag (1968) MR 37:4835

[LM] Lawson H.B. and Michelsohn M.-L.: Spin Geometry, Princeton University Press, Princeton (1989) MR 91g:53001

[MAnn] Mann L. N.: Gaps in the dimensions of transformation groups, Illinois J. Math. 10, 532-546 (1966) MR 34:282

$[\mathrm{Mu} \quad$ Murakami S.: Exceptional simple Lie groups and related topics in recent differential geometry, Differential Geometry and Topology, (Jiang B., Peng Ch.-K., Hou Z., eds.), Lecture Notes in Math. 1369, 183-221, Springer (1989) MR 90g:22009 
[ON1] Oniščik A.L.: Inclusion relations among transitive compact transformation groups, Amer. Math. Soc. Transl. Ser. 2, 50, 5-58 (1966)

[On2] Oniščik A.L.: Topology of transitive transformation groups, Johann Ambrosius Barth, Leipzig (1994)

[PT1] Palais R., Terng C.-L.: A general theory of canonical forms, Trans. Amer. Math. Soc. 300, 771-789 (1987) MR 88f:57069

[PT2] Palais R., Terng C.-L.: Critical Point Theory and Submanifold Geometry, Lecture Notes in Math. 1353, Springer-Verlag, (1988) MR 90c:53143

[PTH] Podestà F., Thorbergsson, G. Polar actions on rank-one symmetric spaces, J. Differential Geom. 53, 131-175 (1999) CMP 2000:16

[TAKAGI] Takagi R.: On homogeneous real hypersurfaces in a complex projective space, Osaka J. Math. 10, 495-506 (1973) MR 49:1433

[TT] Terng C.-L., Thorbergsson G.: Submanifold geometry in symmetric spaces, J. Differential Geom., 42, (1995) MR 97k:53054

[Tits] Tits J.: Tabellen zu den einfachen Lie Gruppen und ihren Darstellungen, Lecture Notes in Math. 40, Springer-Verlag (1967) MR 36:1575

[Wolf] Wolf J. A.: Spaces of constant curvature, Publish or Perish, Wilmington, Delaware (1977)

Institut für Mathematik, Universität Augsburg, 86135 Augsburg, Germany

E-mail address: kollross@math.uni-augsburg.de 